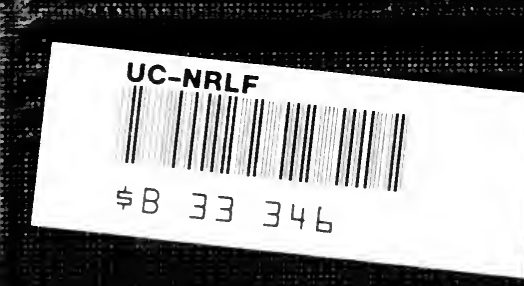

UC-NRLF

"

$\begin{array}{lll}53 & 346\end{array}$

iti 


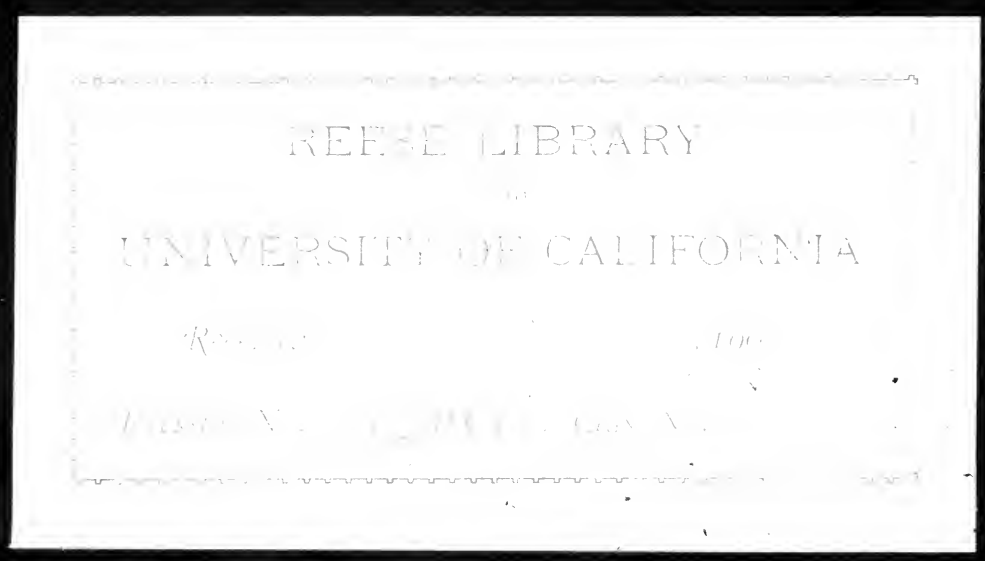







\title{
A COURSE IN
}

\section{MECHANICAL DRAWING.}

BY

JOHN S. REID,

Instructor in Mechanical Drawing and Designing, Sibley Collegre, Cornell University,

Ithaca, $N, Y$.

\begin{abstract}
SECOND EDITION, REVISED. FIRST THOUSAND.
\end{abstract}

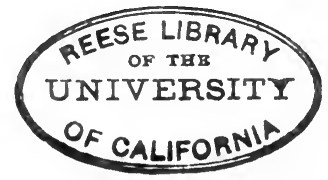

NEW YORK .

JOHN WILEY \& SONS.

London: CHAPMAN \& HALL, Limited.

1900. 
Copyright, $\mathbf{r} 898$,

BY

JOH N S. REID.

ROBERT DRUMMOND, ELECTROTYPBR AND PRINTER, NEW YORK. 


\section{PREFACE.}

IN the course of a large experience as an instructor in drawing and designing, the author of this work has often been called upon to teach the elements of mechanical drawing to students in marine, electrical, railway, and mechanical engineering. Having tried and failed to find a book on the subject that was entirely suitable for his use as a text-book, he has found it necessary to prepare the present work.

This course contains, in the author's judgment, a complete and concise statement, accompanied by examples, of the essential principles of mechanical drawing-all that any young man of ordinary intelligence needs to master, by careful study, the more advanced problems met with in machine construction and design. Such works as the author has tried, although most excellent from certain standpoints, were either incomplete in some of the divisions of the subject or too voluminous and elementary in the treatment of details.

The author does not imagine this work is perfect, but he believes that it comes nearer what is needed in teaching the elements of mechanical drawing in technical schools, high schools, evening drawing schools, and colleges than any work he has examined.

The chapter on Conventions will be appreciated by students 
PREFACE.

when called upon to execute working drawings in practical work. The methods described are considered by the author to be those which have met with general approval by the experienced American draftsmen of the present time.

My acknowledgments are due to E. C. Cleaves, professor of drawing, Sibley College, Cornell University, for reading the manuscript and making some valuable suggestions.

THE Author.

April I, 1898. 


\section{CONTENTS.}

\section{INTRODUCTION.}

The Complete Outfit, Illustrated $\ldots \ldots \ldots \ldots \ldots \ldots \ldots \ldots \ldots \ldots$ i

CHAPTER I.

INSTRUMENTS.................................. 7

Use of Instruments............................

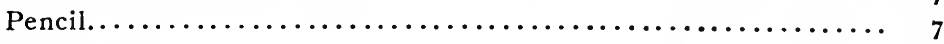

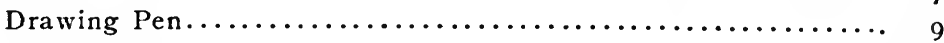

Triangles............................ I

'T Square............................. II

Drawing Board.......................... I

Sibley College Scale........................... 12

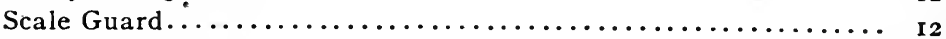

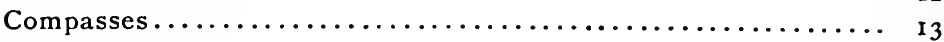

Dividers or Spacers......................... I3

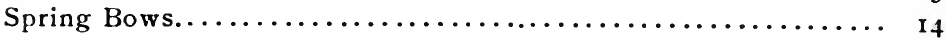

Irregular Curves............................. I4

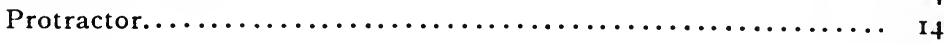

CHAPTER II.

Geometrical Drawing........................... I6

CHAPTER III.

Conventions.................................. 56

CHAPTER IV.

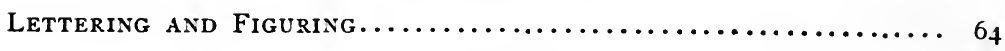

CHAPTER V.

Orthographic Projection........................... 74

Shade Lines, Shades, and Shadows................. ro3

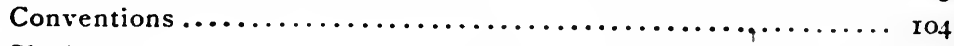

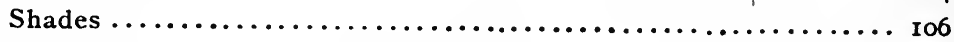

Shadows................................. III

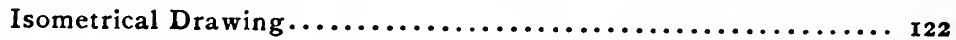

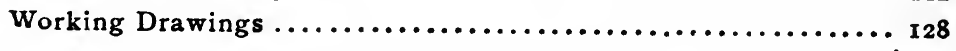





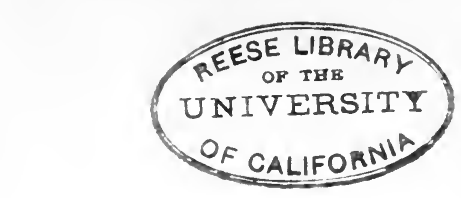

\section{MECHANICAL DRAWING.}

\section{INTRODUCTION.}

A NEED has been felt by instructors and students, especially in technical courses, for a text-book that would illustrate the fundamental principles of mechanical drawing in such a practical, lucid, direct and progressive way as to enable the instructor to teach, and the student to acquire, the greatest number of the essential principles involved, and the ability to apply them, in a draftsman-like manner, in the shortest space of time.

With this in mind, the present work has been prepared from the experience of the writer, a practical draftsman and teacher for over fifteen years.

\section{THE COMPLETE OUTFIT.}

The complete outfit for students in mechanical drawing in Sibley College is as follows:

(I) The DRAwing-BOARD for freshman work is $17^{\prime \prime} \times 22^{\prime \prime}$ $\times \frac{5^{\prime \prime}}{8}$, the same as that used for free-hand drawing. The board for sophomore and junior drawing is $20^{\prime \prime} \times 26^{\prime \prime} \times$ not more than $\frac{7^{\prime \prime}}{2}$ in thickness. The material should be soft pine and constructed as shown by Fig. I. 
(2) PAPER, quality and size to suit.

(3) Pencils, one $6 \mathrm{H}$ and one $4 \mathrm{H} \mathrm{Koh-i-noor} \mathrm{or} \mathrm{Faber,}$ also one Eagle Pilot No. 2 with rubber tip.

(4) The T-SqUARE for freshman work is furnished by the

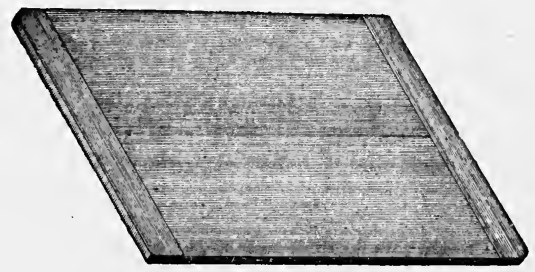

FIG. I.

department; a plain pearwood T-square with a fixed head is all that is necessary for sophomore or junior work. Length to suit drawing-board.

(5) Instruments. "The Sibley College Set," shown by Fig. 2, was compiled by the writer, and is recommended as a first-class medium-priced set of instruments. It contains •

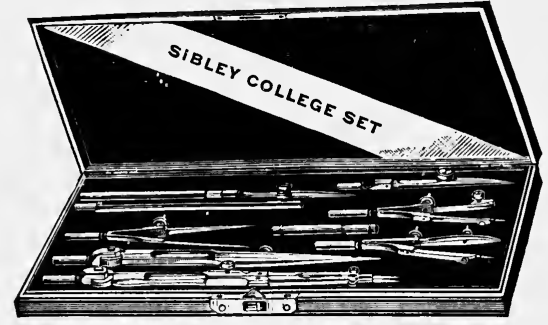

FIG. 2.

A Compass, $5 \frac{1}{2} "$ long, with fixed needle-point, pencil, pen and lengthening bar; a SPRING Bow Pencil, $3^{\prime \prime}$ long; a Spring Bow Pen, 3 "long; a Spring Bow Spacer, 3 "long; a Drawing-PEn, medium length; a HAIR-SPRING Divider, 5 "long; a nickel-plated box with leads.

(6) A Triangular Boxwood Scale graduated as follows : $4^{\prime \prime}$ and $2^{\prime \prime}, 3^{\prime \prime}$ and $\mathrm{I}^{\frac{1}{2}}{ }^{\prime \prime}, \mathrm{I}^{\prime \prime}$, and $\frac{1}{2}^{\prime \prime}, \frac{8}{4}^{\prime \prime}$ and $\frac{3^{\prime \prime}}{8}, \frac{1}{16}{ }^{\prime \prime}$ and $\frac{1}{50}{ }^{\prime \prime}$. 


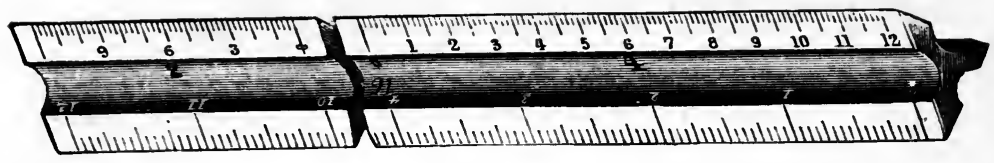

FIG. 3 .
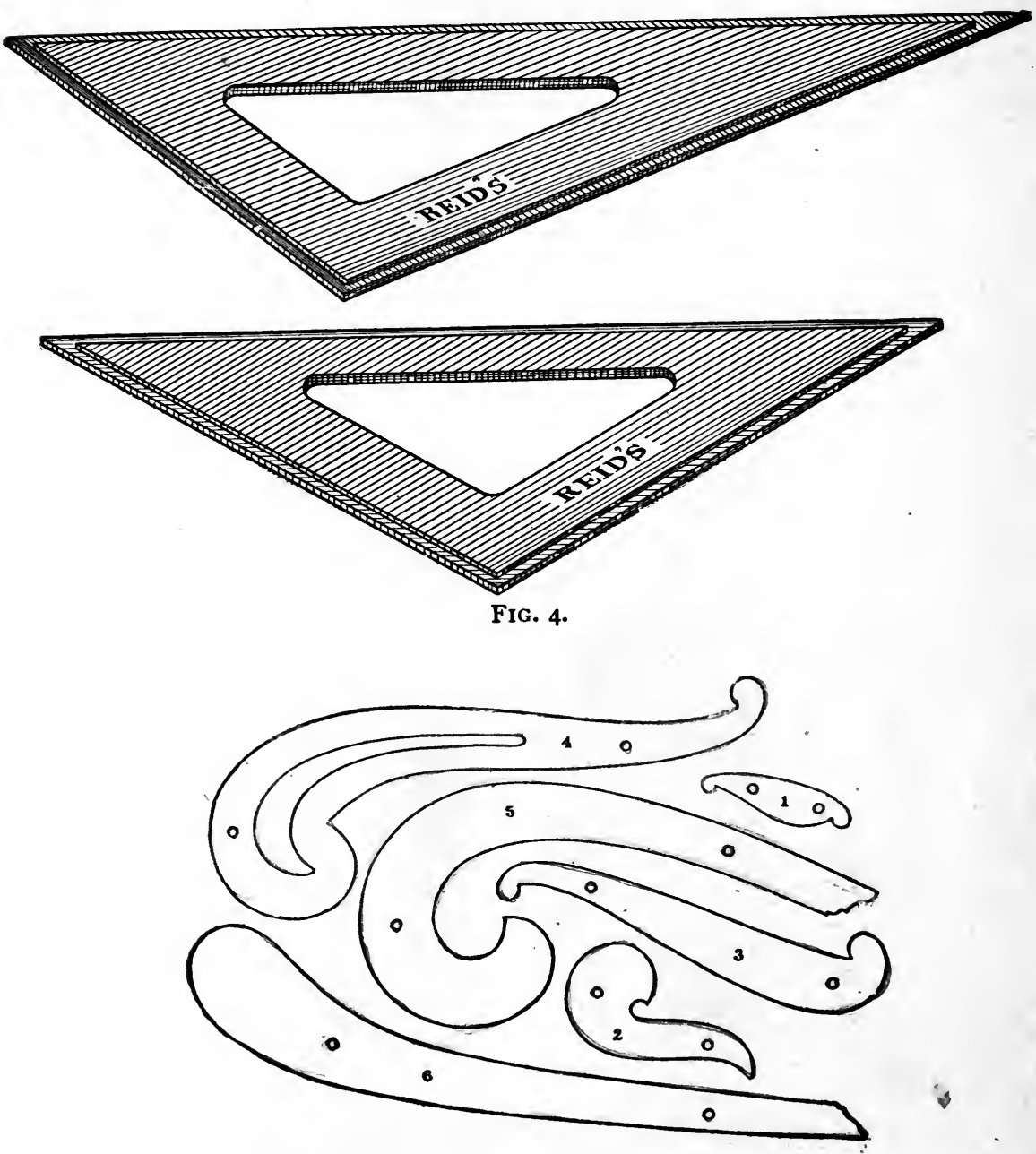

FIG. 5 . 
(7) I TRIANGLE $30^{\circ} \times 60^{\circ}$, celluloid, I $0^{\prime \prime}$ long. Fig. 4. I “ ، $45^{\circ}, \quad 7^{\prime \prime}$ ، “

(8) “Sibley College Set'” of Irregulak Curves. (9) Glass-Paper Pexcil Sharpexer.

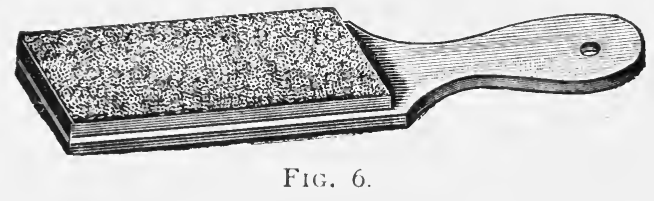

(IO) INk, black waterproof, S.\&H. Fig. 7 . (I I) “ red " " Higgins. Fig. 8. (12) " " blue "

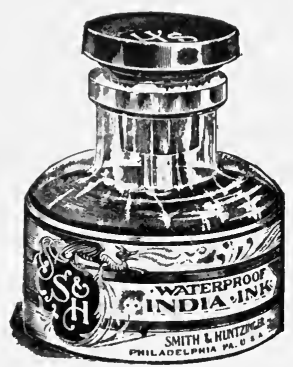

FIG. 7.

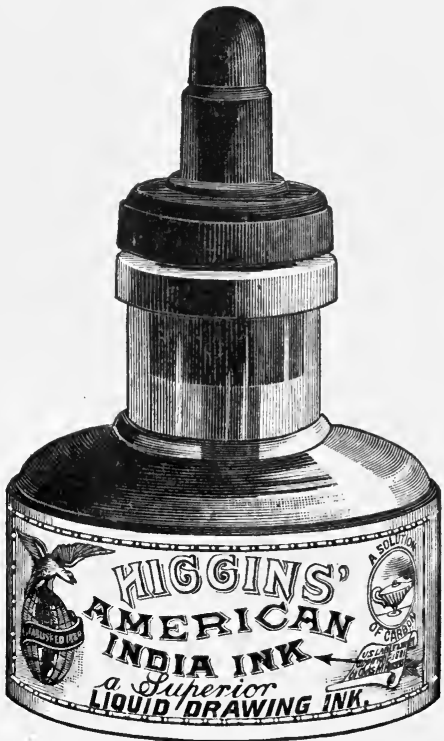

FIG. 8.

(13) Ink Eraser, Faber's Typewriter.

(i4) Pexcil Eraser, Tower's Multiplex Rubber. Fig. 9. 
(i 5 ) Sponge Rubber or Faber's Kneaded Rubber. Fig. 10 .

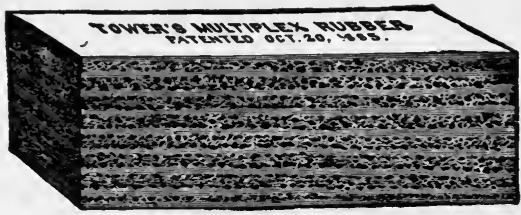

FIG. 9.

(16) TACKs, a small box of $\mathrm{I}$ oz. tacks.

(I 7) WAter-Colors, $\frac{1}{2}$ pan each of Payne's Gray, Crimson Lake, Prussian Blue, Burnt Sienna, and Gamboge. Windsor \& Newton. Fig. II.

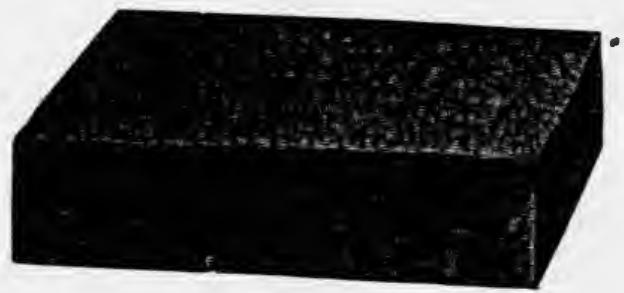

FIG. IO.

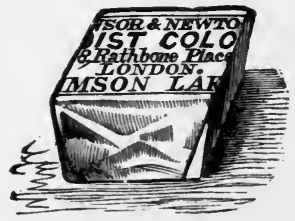

FIG. II.

(i 8) Tinting Brush, Camel's Hair No. Io. Fig. I2.

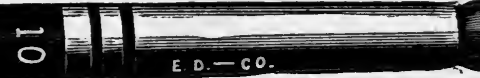

FIG. I2.

(i9) Tinting Saucer. Fig. I3.

(20) Water Glass. Fig. I4.

(2i) Arkansas Oil-stone. $2^{\prime \prime} \times \frac{1}{2} " \times \frac{1}{16} "$.

(22) Piece of Sheet Celluloid No. 300, dull on both sides. Thickness $\frac{5}{1000}$. 
(23) Protractor, German silver, about 5" diam. Fig. I 5. (24) Scale Guard, “ “ Fig. 16.

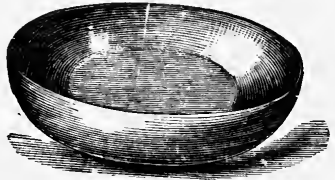

FIG. I3.

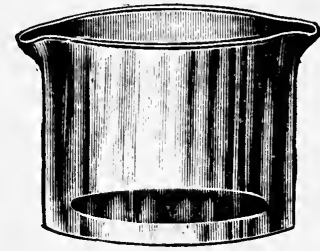

FIG. I4.

(25) Sheet of Tracing-cloth, i $8^{\prime \prime} \times 24^{\prime \prime}$. (26) Writing-PeN, point, "Gillott" No. 303.

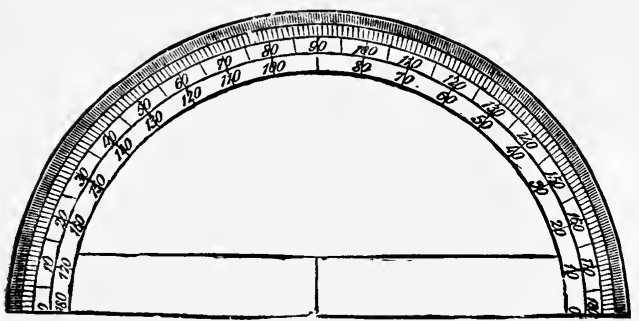

FIG. I5.

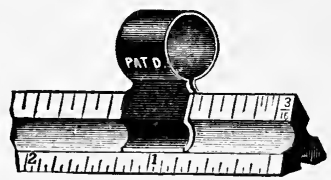

FIG. I6.

(27) Piece of Sheet Brass, $4^{\prime \prime} \times 4^{\prime \prime}$.

(28) NeEdLEs; two with handles.

The following numbers of "The Complete Outfit" are all that the student will be required to purchase for freshman mechanical drawing (No. 2 Register, '97-'98): 2, 3, 5, 6, 7, 8,9, I0, I 3, I $4,16,26$.

The remainder of the outfit may be purchased during the sophomore and junior years. 


\section{CHAPTER I.}

\section{INSTRUMENTS.}

IT is a common belief among students that any kind of cheap instrument will do with which to learn mechanical drawing, and not until they have acquired the proper use of the instruments should they spend money in buying a firstclass set. This is one of the greatest mistakes that can be made. Many a student has been discouraged and disgusted because, try as he would, he could not make a good drawing, using a set of instruments with which it would be difficult for even an experienced draftsman to make a creditable showing.

If it is necessary to economize in this direction it is better and easier to get along with a fewer number, and have them of the best, than it is to have an elaborate outfit of questionable quality.

The instruments composing the "Sibley College Set" are made by T. Alteneder \& Sons, and are certainly as good as the best. See Fig. 17 .

USE OF INSTRUMENTS.

The Pencil.-Designs of all kinds are usually worked out in pencil first, and if to be finished and kept they are inked in and sometimes colored and shaded; but if the drawing is only to be finished in pencil, then all the lines except construction, center, and dimension lines should be made broad and dark, 
so that the drawing will stand out clear and distinct. It will be noticed that this calls for two kinds of pencil-lines, the first a thin, even line made with a hard, fine-grained leadpencil, not less than $6 \mathrm{H}$ (either Koh-i-noor or Faber's), and sharpened to a knife-edge in the following manner: The lead should be carefully bared of the wood with a knife for about $\frac{1}{2}{ }^{\prime \prime}$, and the wood neatly tapered back from that point; then lay the lead upon the glass-paper sharpener illustrated in the outfit, and carefully rub to and fro until the pencil assumes a long taper from the wood to the point; now turn it over and do the same with the other side, using toward the last a slightly oscillating motion on both sides until the point has assumed a sharp, thin, knife-edge endwise and an elliptical contour the other way.

This point should then be polished on a piece of scrap drawing-paper until the rough burr left by the glass-paper is removed, leaving a smooth, keen, ideal pencil-point for drawing straight lines.

With such a point but little pressure is required in the hands of the draftsman to draw the most desirable line, one that can be easily erased when necessary and inked in to much better advantage than if the line had been made with a blunt point, because, when the pencil-point is blunt the inclination is to press hard upon it when drawing a line. This forms a groove in the paper which makes it very difficult to draw an even inked line.

The second kind of a pencil-line is the broad line, as explained above; it should be drawn with a somewhat softer pencil, say $4 \mathrm{H}$, and a thicker point.

All lines not necessary to explain the drawing should be 
erased before inking or broadening the pencil-lines, so as to make a minimum of erasing and cleaning after the drawing is finished.

When drawing pencil-lines, the pencil should be held in a plane passing through the edge of the $\mathrm{T}$-square perpendicular to the plane of the paper and making an angle with the plane of the paper equal to about $60^{\circ}$.

Lines should always be drawn from left to right. A soft conical-pointed pencil should be used for lettering, figuring, and all free-hand work.

The Drawing-pen.- The best form, in the writer's opinion, is that shown in Fig. I7. The spring on the upper blade

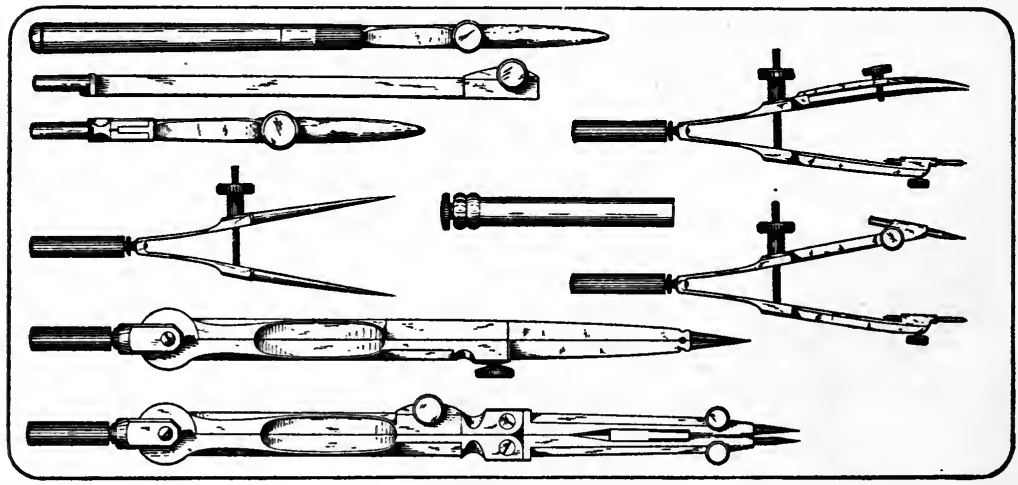

FIG. 17 .

spreads the blades sufficiently apart to allow for thorough cleaning and sharpening. The hinged blade is therefore unnecessary. The pen should be held in a plane passing through the edge of the $\mathrm{T}$-square at right angles to the plane of the paper, and making an angle with the plane of the paper ranging from $60^{\circ}$ to $90^{\circ}$.

The best of drawing-pens will in time wear dull on the point, and until the student has learned from a competent 
teacher how to sharpen his pens it would be better to have them sharpened by the manufacturer.

It is difficult to explain the method of sharpening a drawing-pen.

If one blade has worn shorter than the other, the blades should be brought together by means of the thumb-screw, and placing the pen in an upright position draw the point to and fro on the oil-stone in a plane perpendicular to it, raising and lowering the handle of the pen at the same time, to give the proper curve to the point. The Arkansas oil-stones (No. 2 I of "The Complete Outfit") are best for this purpose.

The blades should next be opened slightly, and holding the pen in the right hand in a nearly horizontal position, place the lower blade on the stone and move it quickly to and fro, slightly turning the pen with the fingers and elevating the handle a little at the end of each stroke. Having ground the lower blade a little, turn the pen completely over and grind the upper blade in a similar manner for about the same length of time; then clean the blades and examine the extreme points, and if there are still bright spots to be seen continue the grinding until they entirely disappear, and finish the sharpening by polishing on a piece of smooth leather.

The blades should not be too sharp, or they will cut the paper. The grinding should be continued only as long as the bright spots show on the points of the blades.

When inking, the pen should be held in about the same position as described for holding the pencil. Many draftsmen hold the pen vertically. The position may be varied with good results as the pen wears. Lines made with the pen should only be drawn from left to right. 


\section{THE TRIANGLES.}

The triangles shown at Fig. 4 (in " The Complete Outfit") are $1 \mathrm{O}^{\prime \prime}$ and $7^{\prime \prime}$ long respectively, and are made of transparent celluloid. The black rubber triangles sometimes used are but very little cheaper (about Io cents) and soon become dirty when in use; the rubber is brittle and more easily broken than the celluloid.

Angles of $15^{\circ}, 75^{\circ}, 30^{\circ}, 45^{\circ}, 60^{\circ}$, and $90^{\circ}$ can readily be drawn with the triangles and $T$-square. Lines parallel to oblique lines on the drawing can be drawn with the triangles by placing the edge representing the height of one of them so as to coincide with the given line, then place the edge representing the hypotenuse of the other against the corresponding edge of the first, and by sliding the upper on the lower when holding the lower firmly with the left hand any number of lines may be drawn parallel to the given line.

The methods of drawing perpendicular lines and making angles with other lines within the scope of the triangles and $T$. square are so evident that further explanation is unnecessary.

THE T-SQUARE.

The use of the $T$-square is very simple, and is accomplished by holding the head firmly with the left hand against the left-hand end of the drawing-board, leaving the right hand free to use the pen or pencil in drawing the required lines.

THE DRAIVING-BOARD.

If the left-hand edge of the drawing-board is straight and even and the paper is tacked down square with that edge and 
the $\mathrm{T}$-square, then horizontal lines parallel to the upper edge of the paper and perpendicular to the left-hand edge may be drawn with the $\mathrm{T}$-square, and lines perpendicular to these can be made by means of the triangles, or set squares, as they are sometimes called.

THE SIbley COLLEGE SCALE.

This scale, illustrated in Fig. 3 (in " The Complete Outfit"), was arranged to suit the needs of the students in Sibley College. It is triangular and made of boxwood. The six edges are graduated as follows; $\frac{1}{16}{ }^{\prime \prime}$ or full size, $\frac{1}{5}{ }^{\prime \prime}, \frac{3}{4}{ }^{\prime \prime}$ and $\frac{3^{\prime \prime}}{8}=\mathrm{I} \mathrm{ft} ., \mathrm{I}^{\prime \prime}$ and $\frac{1}{2}^{\prime \prime}=\mathrm{I} \mathrm{ft} ., 3^{\prime \prime}$ and $\mathrm{I}_{2}{ }^{\prime \prime}=\mathrm{I} \mathrm{ft}$. , and $4^{\prime \prime}$ and $2^{\prime \prime}=\mathrm{I} \mathrm{ft}$.

Drawings of very small objects are generally shown enlarged-e.g., if it is determined to make a drawing twice the full size of an object, then where the object measures one inch the drawing would be made $2^{\prime \prime}$, etc.

Larger objects or small machine parts are often drawn full size-i.e., the same size as the object really is-and the drawing is said to be made to the scale of full size.

Large machines and large details are usually made to a reduced scale-e.g., if a drawing is to be made to the scale of $2^{\prime \prime}=\mathrm{I} \mathrm{ft}$., then $2^{\prime \prime}$ measured by the standard rule would be divided into 12 equal parts and each part would represent $\mathrm{I}^{\prime \prime}$. See Fig. $8 \mathrm{I} b$.

THE SCALE GUARD.

This instrument is shown in Fig. I6 (in "The Complete Outfit"). It is employed to prevent the scale from turning, so that the draftsman can use it without having to look for 
the particular edge he needs every time he wants to lay off a measurement.

THE COMPASSES.

When about to draw a circle or an arc of a circle, take hold of the compass at the joint with the thumb and two first fingers, guide the needle-point into the center and set the pencil or pen leg to the required radius, then move the thumb and forefinger up to the small handle provided at the top of the instrument, and beginning at the lowest point draw the line clockwise. The weight of the compass will be the only down pressure required.

The sharpening of the lead for the compasses is a very important matter, and cannot be emphasized too much. Before commencing a drawing it pays well to take time to properly sharpen the pencil and the lead for compasses and to keep them always in good condition.

The directions for sharpening the compass leads are the same as has already been given for the sharpening of the straight-line pencil.

\section{THE DIVIDERS OR SPACERS.}

This instrument should be held in the same manner as described for the compass. It is very useful in laying off equal distances on straight lines or circles. To divide a given line into any number of equal parts with the dividers, say 12 , it is best to divide the line into three or four parts first, say 4 , and then when one of these parts has been subdivided accurately into three equal parts, it will be a simple matter to step off these latter divisions on the remaining three-fourths 
of the given line. Care should be taken not to make holes in the paper with the spacers, as it is difficult to ink over them without blotting.

\section{THE SPRING BOWS.}

These instruments are valuable for drawing the small circles and arcs of circles. It is very important that all the smali arcs, such as fillets, round corners, etc., should be carefully pencilled in before beginning to ink a drawing. Many good drawings are spoiled because of the bad joints between small arcs and straight lines.

When commencing to ink a drawing, all small arcs and small circles should be inked first, then the larger arcs and circles, and the straight lines last. This is best, because it is much easier to know where to stop the arc line, and to draw the straight line tangent to it, than vice versa.

\section{IRREGULAR CURVES.}

The Sibley College Set of Irregular Curves shown in Fig. 5 are useful for drawing irregular curves through points that have already been found by construction, such as ellipses, cycloids, epicyloids, etc., as in the cases of gear-teeth, cam outlines, rotary pump wheels, etc.

When using these curves, that curve should be selected that will coincide with the greatest number of points on the line required.

THE PROTRACTOR.

This instrument is for measuring and constructing angles. It is shown in Fig. I 5. It is used as follows when measuring 
an angle: Place the lower straight edge on the straight line which forms one of the sides of the angle, with the nick exactly on the point of the angle to be measured. Then the number of degrees contained in the angle may be read from the left, clockwise.

In constructing an angle, place the nick at the point from which it is desired to draw the angle, and on the outer circurnference of the protractor, find the figure corresponding to the number of degrees in the required angle, and mark a point on the paper as close as possible to the figure on the protractor; after removing the protractor, draw a line through this point to the nick, which will give the required angle. 


\section{CHAPTER II.}

\section{GEOMETRICAL DRAWING.}

The following problems are given to serve a double purpose: to teach the use of drawing instruments, and to point out those problems in practical geometry that are most useful in mechanical drawing, and to impress them upon the mind of the student so that he may readily apply them in practice.

The drawing-paper for this work should be divided temporarily, with light pencil-lines, into as many squares and rectangles as may be directed by the instructor, and the drawings made as large as the size of the squares will permit. The average size of the squares should be not less than $4^{\prime \prime}$. When a sheet of drawings is finished these boundary lines may be erased.

It will be noticed in the illustrations of this chapter that all construction lines are made very narrow, and given and required lines quite broad. This is sufficient to distinguish them, and employs less time than would be necessary if the construction lines were made broken, as is often the case.

If time will permit, it is advisable to ink in some of these drawings toward the last. In that event, the given lines may be red, the construction lines blue, and the required lines black.

But even when inked in in black, the broad and narrow 
lines would serve the purpose very well without the use of colored inks.

The principal thing to be aimed at in making these drawings is accuracy of construction. All dimensions should be laid off carefully, correctly, and quickly. Straight lines joining arcs should be exactly tangent, so that the joints cannot be noticed. It is the little things like these that make or mar a drawing, and if attended to or neglected they will make or mar the draftsman. The constant endeavor of the student should be to make every drawing he begins more accurate, quicker and better in every way than the preceding one.

A drawing should never be handed in as finished until the student is perfectly sure that he cannot improve it in any way whatever, for the act of handing in a drawing is the same, or should be the same, as saying This is the best that I can do; I cannot improve it; it is a true measure of my ability to make this drawing.

If these suggestions are faithfully followed throughout this course, success awaits any one who earnestly desires it.

Fig. i8. To Bisect a Finite Straight Line.-With $A$ and $B$ in turn as centers, and a radius greater than the half of $A B$, draw arcs intersecting at $E$ and $F$. Join $E F$ bisecting $A B$ at $C$.

An arc of a circle may be bisected in the same way.

Fig. 19. To Erect a Perpendicular at the End of THE LINE.-Assume the point $E$ above the line as center and radius $E B$ describe an arc $C B D$ cutting the line $A B$ in the point $C$. From $C$ draw a line through $E$ cutting the arc in $D$. Draw $D B$ the perpendicular.

Fig. 20. The Same Problem: a Second Method.- 
With center $B$ and any radius as $B C$ describe an arc $C D E$ with the same radius; measure off the $\operatorname{arcs} C D$ and $D E$. With $C$ and $D$ as centers and any convenient radius describe arcs intersecting at $F . F B$ is the required perpendicular.

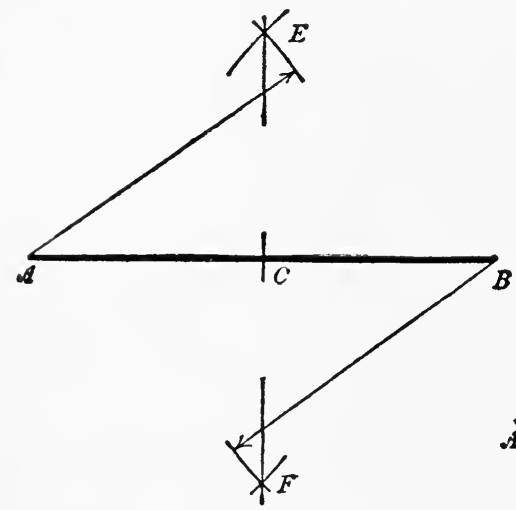

FIG. IS.

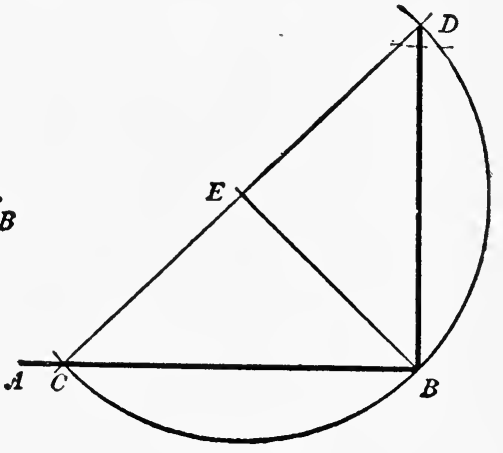

FIG. I9.

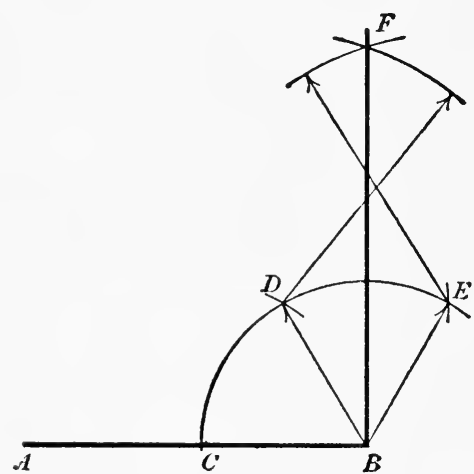

Fig. 20.

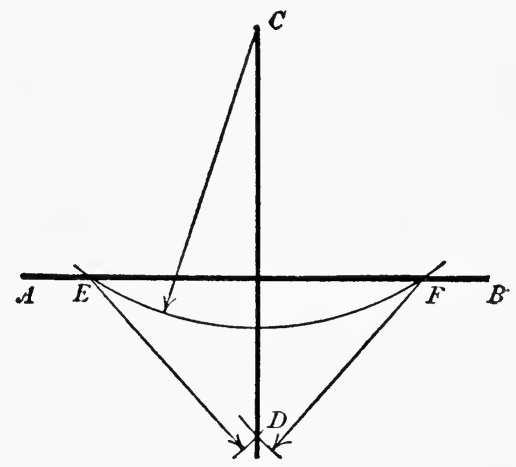

FIG. 21 .

Fig. 2 I. To Draw a Perpendicular to a Line From a POIN ABOVE OR Below IT.-Assume the point $C$ above the line. With center $C$ and any suitable radius cut the line $A B$ in $E$ and $F$. From $E$ and $F$ describe arcs. cutting in $D$. Draw $C D$ the perpendicular required. 
Fig. 22. To Bisect a Given Angle.-With $A$ as center and any convenient radius describe the arc $B C$. With $B$ and $C$ as centers and any convenient radius draw arcs intersecting at $D$. Join $A D$, then angle $B A D=$ angle $D A C$.

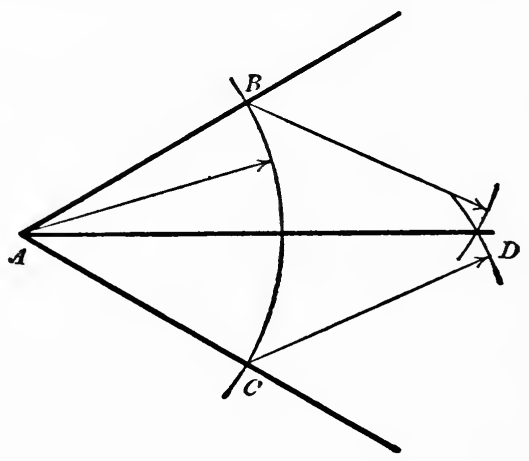

FIG. 22.

Fig. 23. To Draw a Line Parallel to a Given I.ine $A B$ Through a Given Point $C$.-From any point on $A B$ as $B$ with radius $B C$ describe an arc cutting $A B$ in $A$. From $C$ with the same radius describe arc $B D$. From $B$ with $A C$ as radius cut arc $B D$ in $D$. Draw $C D$. Line $C D$ is parallel to $A B$.

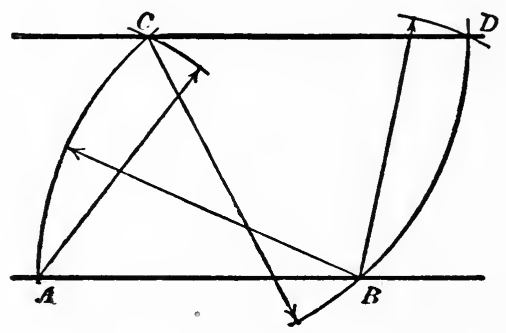

FIG. 23.

Fig. 24. From a Point $D$ on the Line $D E$ to set off an Angle equal to the given Angle $B A C$.-From 
$A$ with any convenient radius describe arc $B C$. From $D$ with the same radius describe arc $E F$. With center $E$ and radius $B C$ cut arc $E F$ in $F$. Join $D F$. Angle $E D F$ is = angle $B A C$.

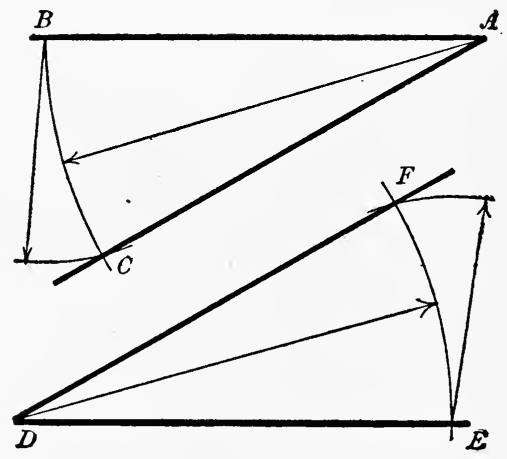

FIG. 24 .

Fig. 25. To Divide an ANgle into two EQUai, Parts, when the Lines do not Exteni to a Meeting Point.-Draw the line $C D$ and $C E$ parallel and at equal dis-

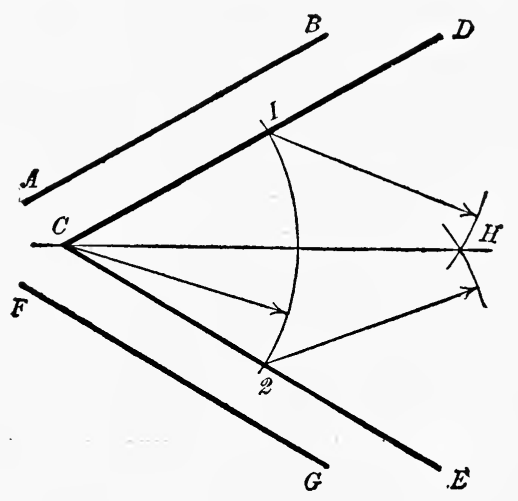

FIG. 25.

tances from the lines $A B$ and $F G$. With $C$ as center and any radius draw arcs $I, 2$. With $I$ and 2 as centers and any con- 
venient radius describe arcs intersecting at $H$. A line through $C$ and $H$ divides the angle into two equal parts.

Fig. 26. To Construct a Rhomboid having AdjaCent Sides equal to two Given Lines $A B$ and $A C$, and an Axgle equal to a Given Axgle $A$.-Draw line $D E$ equal to $A B$. Make $D=$ angle $A$. Make $D F=A C$. From $F$ with line $A B$ as radius and from $E$ with line $A C$ as radius describe arcs cutting in $G$. Join $F G$ and $E G$.

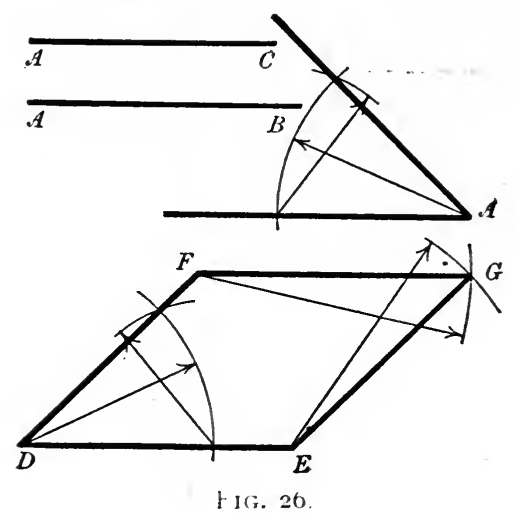

Fig. 27. To Divide the Line $A B$ into any Number OF EqUal PARTS, SAY 15.-Draw a line $C D$ parallel to $A B$, of any convenient length. From $C$ set off along this line the number of equal parts into which the line $A B$ is to be divided. Draw $C A$ and $D B$ and produce them until they intersect at $E$. Through each one of the points I, 2, 3, 4, etc., draw lines to the point $E$, dividing the line $A B$ into the required number of equal parts.

This problem is useful in dividing a line when the point required is difficult to find accurately-e.g., in Fig. $28 A B$ is the pitch of the spur gear, partly shown, which includes a 
space and a tooth and is measured on the pitch circle. In cast gears the space is made larger than the thickness of the tooth, the proportion being about 6 to 5 -i.e., if we divide the pitch into eleven equal parts the space will measure $\frac{6}{11}$

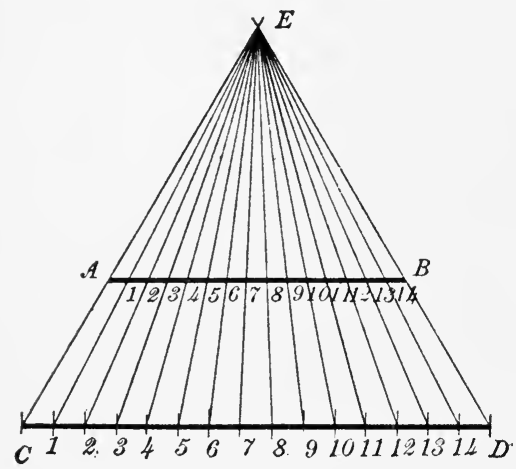

Fic. 27.

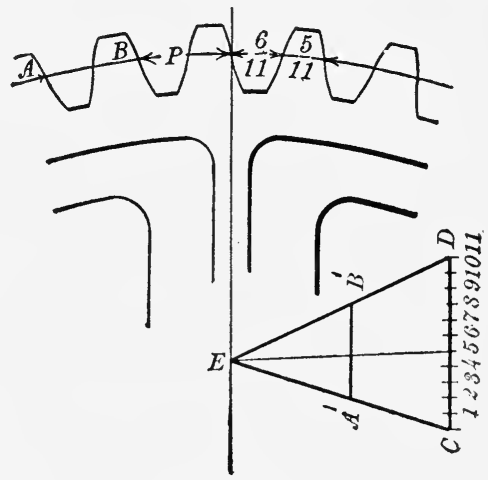

FIG 28.

and the tooth $\frac{5}{11}$. The $\frac{1}{11}$ which the space is larger than the tooth is called the backlash. Let $A^{\prime} B^{\prime}$ be the pitch chord of the arc $A B$. Draw $C D$ parallel to $A^{\prime} B^{\prime}$ at any convenient distance and set off on it I I equal spaces of any convenient length. Draw $C A^{\prime}$ and $D B^{\prime}$ intersecting at $E$. From point 5 draw a line to $E$ which will divide $A^{\prime} B^{\prime}$ as required; the one part $\frac{5}{11}$ and the other $\frac{6}{11}$.

Fig. 29. To Divide a Given Line into Axy Number of Equal Parts: Another Methon.-Let $A B$ be the given line. From $A$ draw $A C$ at any angle, and lay off on it the required number of equal spaces of any convenient length. Join $C B$ and through the divisions on $A C$ draw lines parallel to $C B$, dividing $A B$ as required in the points $\mathbf{I}^{\prime}, 2^{\prime}, 3^{\prime}, 4^{\prime}$, etc.

Fig. 3o. To Divide a Line $A B$ Proportionally to The Dividen Line $C D$. - Draw $A B$ parallel to $C D$ at any 
distance from it. Draw lines through $C A$ and $D B$ and produce them till they meet at $E$. Draw lincs from $E$ through the divisions I, 2, 3, 4, etc., of line $C D$, cutting line $A B$ in the

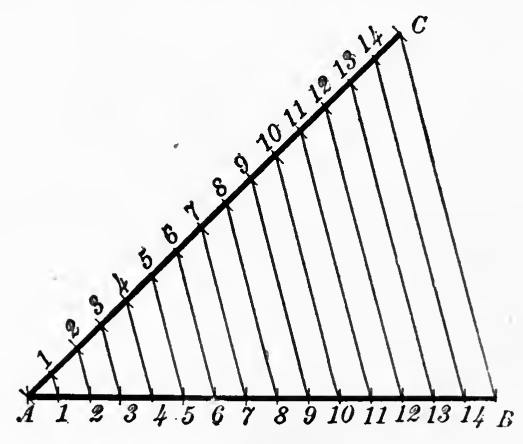

FIG. 29.

points $5,6,7,8$, etc. The divisions on $A B$ will have the same proportion to the divisions on $C D$ that the whole line $A B$ has to the whole line $C D$-i.e., the lines will be proportionally divided.

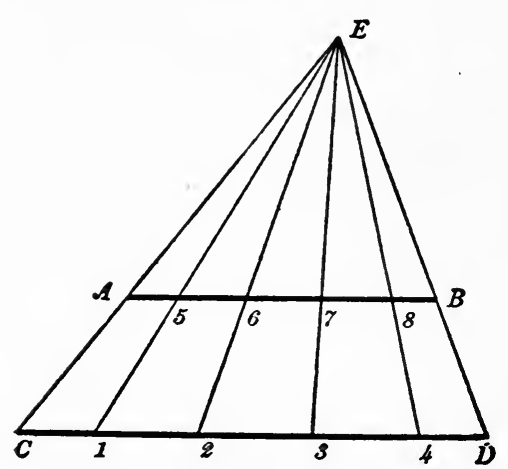

FIG. 30 .

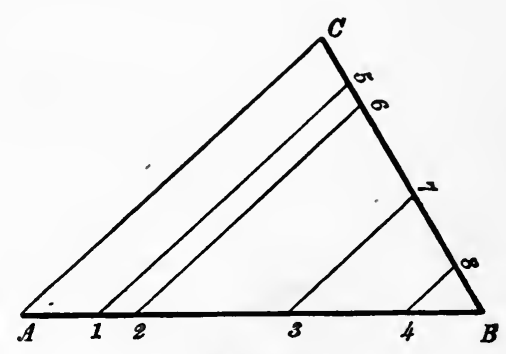

Fig. 3r.

Fig. 3i. The Same: Another Method.-Let $B C$, the divided line, make any angle with $B A$, the line to be di- 
vided at $B$. Draw line $C A$ joining the two ends of the lines. Draw lines from $5,6,7,8$, parallel to $C A$, dividing line $A B$ in points $\mathrm{I}, 2,3,4$, proportional to $B C$.

Fig. 32. To Construct an Equilateral Triangle ON a Given Base $A B$.-From the points $A$ and $B$ with $A B$ as radius describe arcs cutting in $C$. Draw lines $A C$ and $B C$. The triangle $A B C$ is equilateral and equiangular.

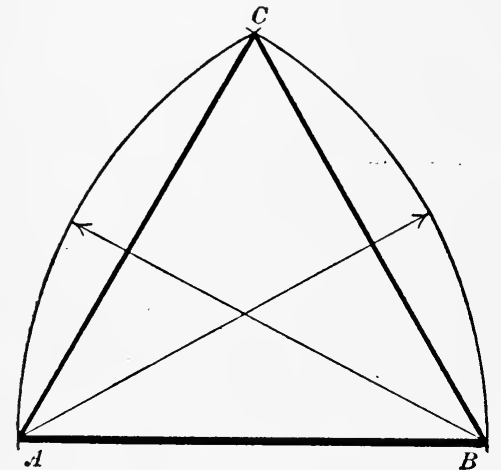

FIG. 32.

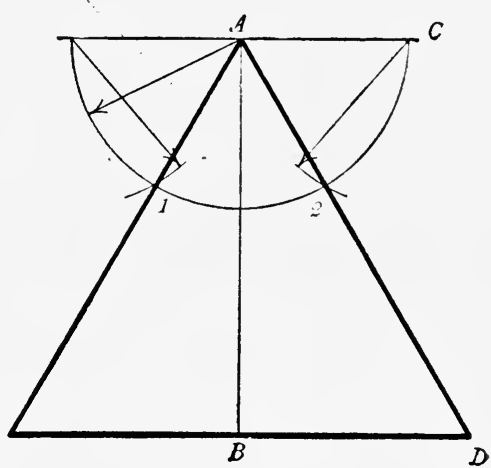

FIG. 33.

Fig. 33. To Construct an Equilateral Triangle of a Given Altitude, $A B$.-From both ends of $A B$ draw lines perpendicular to it as $C A$ and $D B$. From $A$ with any radius describe a semicircle on $C A$ and with its radius cut off arcs 1,2. Draw lines from $A$ through 1, 2, and produce them until they cut the base $B D$.

Fig. 34. To Trisect a Right Angle $A B C$.- From the angular point $B$ with any convenient radius describe an arc cutting the sides of the angle in $C$ and $A$. From $C$ and $A$ with the same radius cut off arcs I and 2. Draw lines I $B$ and $2 B$, and the right angle will be trisected. 
Fig. 35. To Construct any Triangle, its Three Sides $A B$ AND $C$ BEING GIVEN.-From one end of the base as $A$ describe an arc with the line $B$ as radius. From the other end with line $C$ as radius describe an arc, cutting the first arc in $D$. From $D$ draw lines to the ends of line $A$, and a triangle will be constructed having its sides equal to the sides given. To construct any triangle the two shorter sides $B$ and $C$ must together be more than equal to the largest side $A$.

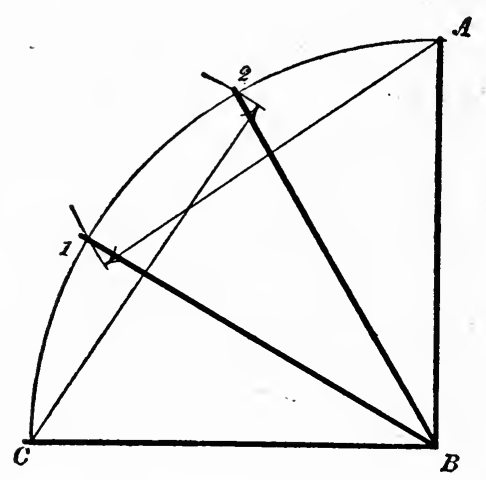

FIG. 34 .

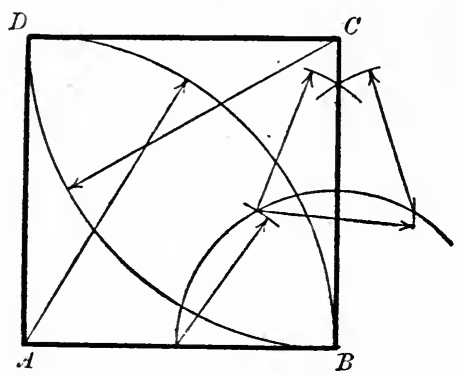

FIG. 36 .

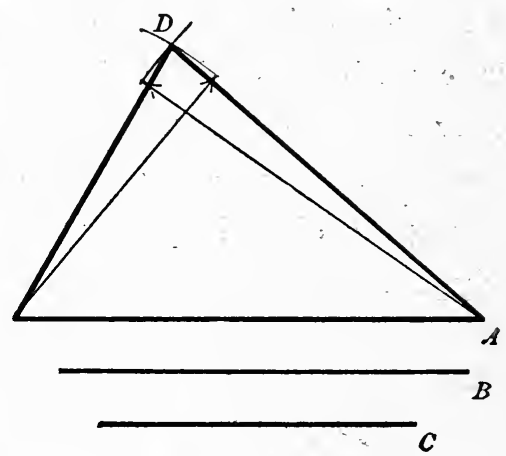

Fla. 35.

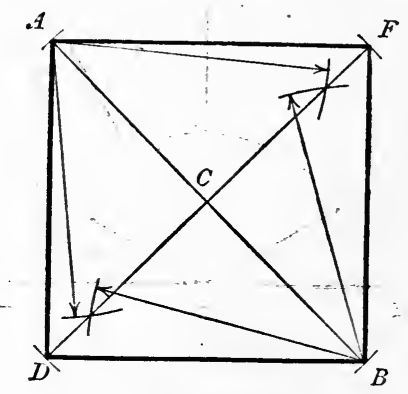

- FIG. 37.

Fig. 36. To Construct a Square, its Base $A B$ BEING GIVEN.-Erect a perpendicular at $B$. Make $B C$ equal 
to $A B$. From $A$ and $C$ with radius $A B$ describe arcs cutting in $D$. Join $D C$ and $D A$.

Fig. 37. To Construc't a Square, given its Diagonal $A B$. - Bisect $A B$ in $C$. Draw $D F$ perpendicular to $A B$ at $C$. Make $C D$ and $C F$ each equal to $C A$. Join $A D$, $D B, B F$, and $F A$.

Fig. 38. To Construct a Regular Polygon of any Number of Sides, the Circumscribing Circle being GIVEN.-At any point of contact, as $C$, draw a tangent $A B$ to the given circle. From $C$ with any radius describe a semicircle cutting the given circle. Divide the semicircle into as many equal parts as the polygon is required to have sides, as I, 2, 3, 4, 5, 6. Draw lines from $C$ through each division, cutting the circle in points which will give the angles of the polygon.

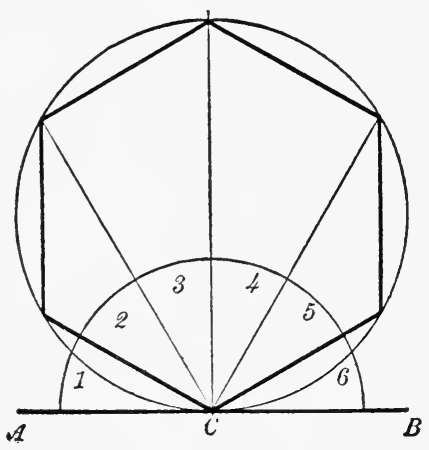

FIG. 39 .

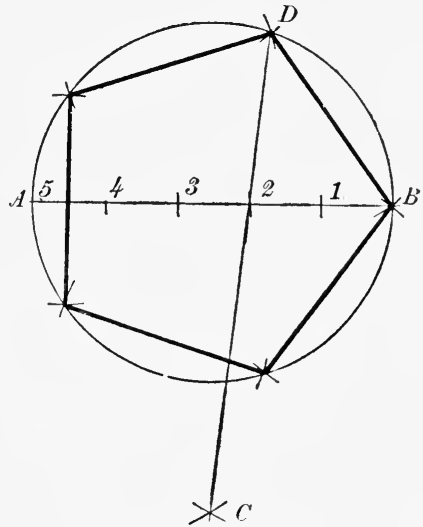

FIG. 39.

Fig. 39. Another Method.-Draw a diameter $A B$ of the given circle. Divide $A B$ into as many equal parts as the polygon is to have sides, say 5. From $A$ and $B$ with the 
line $A B$ as radius describe arcs cutting in $C$, draw a line from $C$ through the second division of the diameter and produce it cutting the circle in $D . \quad B D$ will be the side of the required polygon. The line $C$ must always be drawn through the second division of the diameter, whatever the number of sides of the polygon.

Fig. 40. To Construct any Regular Polygon With A Given Side $A B$.- Make $B D$ perpendicular and equal to $A B$. With $B$ as center and radius $A B$ describe arc $D A$. Divide arc $D A$ into as many equal parts as there are sides in the required polygon, as $1,2,3,4,5$. Draw $B 2$. Bisect line $A B$ and erect a perpendicular at the bisection cutting $B 2$ in $C$. With $C$ as center and radius $C B$ describe a circle. With $A B$ as a chord step off the remaining sides of the polygon.

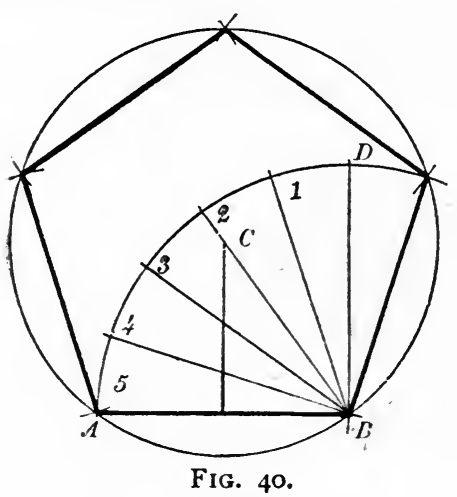

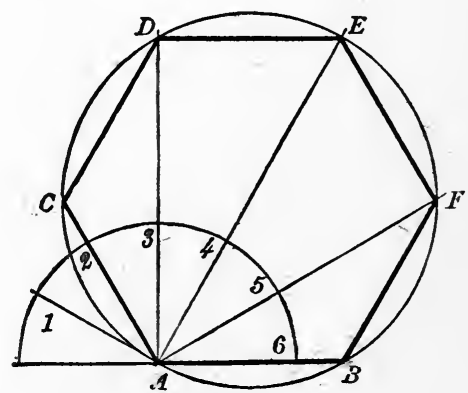

FIG. 4 I.

Fig. 4I. Another Method.-Extend line $A \underline{B}$. With center $A$ and any convenient radius describe a semicircle. Divide the semicircle into as many equal parts as there are sides in the required polygon, say 6. Draw lines through every division except the first. With $A$ as center and $A B$ as 
radius cut off $A 2$ in $C$. From $C$ with the same radius cut $A 3$. in $D$. From $D, A_{4}$ in $E$. From $B, A_{5}$ in $F$. Join $A C, C D$, $D E ; E F$, and $F B$.

Fig. 42. To Construct a Regular Heptagon, the: Circumscribing Circle being given.-Draw a radius $A B$. With $B$ as center and $B A$ as radius, cut the circumference in I, 2 ; it will be bisected by the radius in $C_{1} C_{1}$ or $C_{2}$ is equal to the side of the required heptagon.

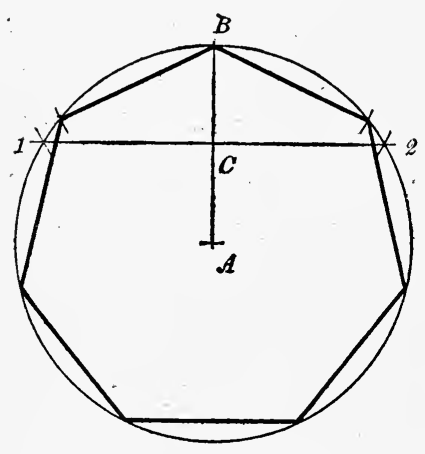

FIG. 42.

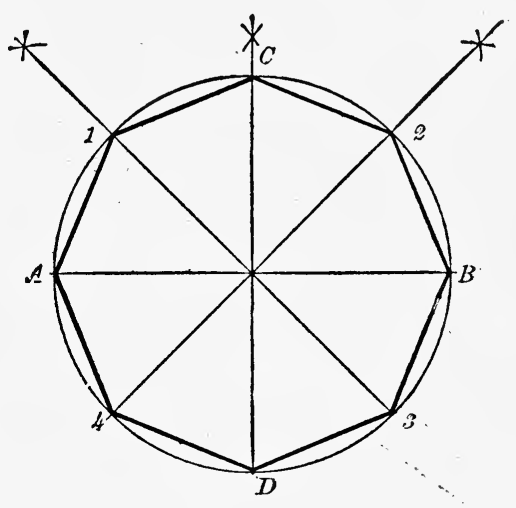

FIG 43.

Fig. 43. To Construct a Regular Octagon, the Circumscribing Circle being given.-Draw a diameter $A B$. Bisect the $\operatorname{arcs} A B$ in $C$ and $D$. Bisect $\operatorname{arcs} C A$ and $C B$ in $\mathrm{I}$ and 2. Draw lines from $\mathrm{I}$ and 2 through the center of the circle, cutting the circumference in 3 and 4 . Join $A \mathrm{I}$, I $C, C_{2}, 2 B, B 3$, etc.

Fig. 44. To Construct a Pentagon, the Side $A B$ BeIng Given.-Produce $A B$. With $B$ as center and $B A$ as radius, describe arc $A D_{2}$. With center $A$ and same radius, describe an arc cutting the first $\operatorname{arc}$ in $D$. Bisect $A B$ in $E$. 
Draw line $D E$. Bisect arc $B D$ in $F$. Draw line $E F$. With center $C$ and radius $E F$ cut off arc $C_{\mathrm{I}}$ and $\mathrm{I}, 2$ on the semicircle. Draw line $B 2$; it will be a second side of the penta-

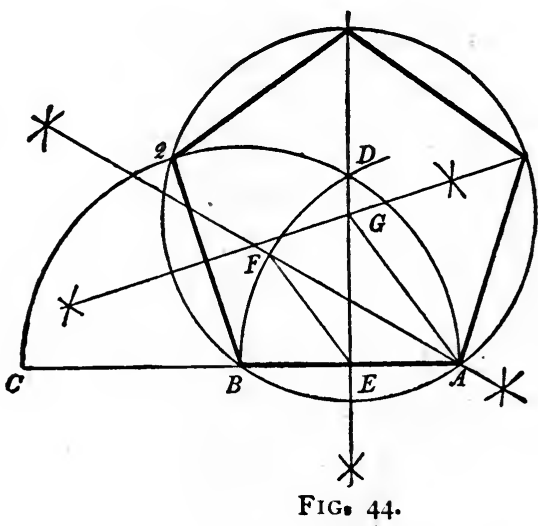

gon. Bisect it and draw a line perpendicular to it at the bisection. The perpendiculars from the sides $A B$ and $B 2$ will cut in $G$. With $G$ as center and radius $G A$ describe a circle $\cdot$ it will contain the pentagon.

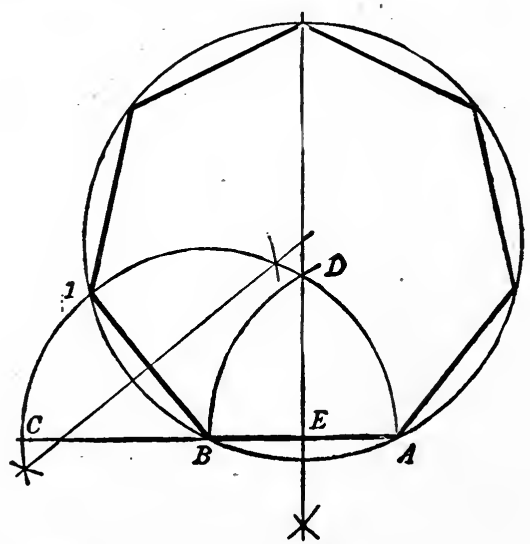

Fig. 45. 
Fig. 45. To Construct a Heptagon on a Given Line $A B$. - Extend line $A B$ to $C$. From $B$ with radius $A B$ describe a semicircle. With center $A$ and same radius describe an arc cutting the semicircle in $D$. Bisect $A B$ in $E$. Draw line $D E$. With $C$ as center and $D E$ as radius, cut off arc $I$ on the semicircle. Draw line $B \mathrm{I}$; it is a second side of the heptagon. Bisect it and obtain the center of the circumscribing circle as in the preceding problem.

Fig. 46. To Inscribe ax Octagon in a Given SQUARE.-Draw diagonals $A D, C B$ intersecting at $O$. From $A, B, C$, and $D$ with radius equal to $A O$ describe quadrants cutting the sides of the square in $1,2,3,4,5,6,7,8$. Join these points and the octagon will be inscribed.

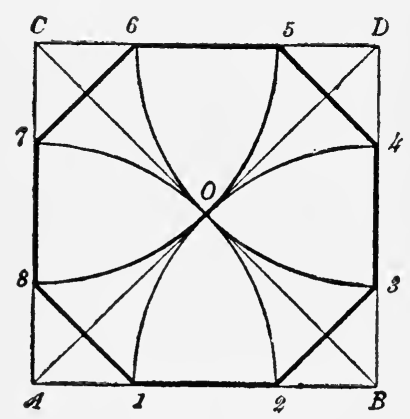

FIG. 46.

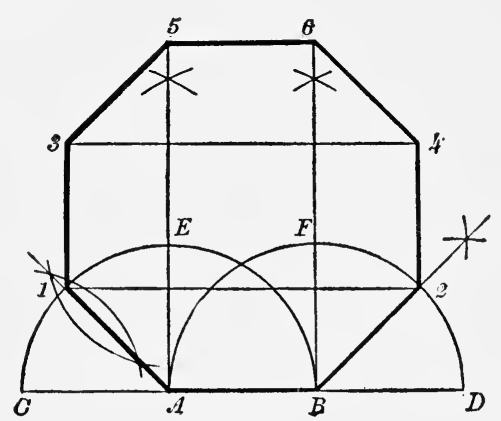

FIG. 47 .

Fig, 47. To Construct a Regular Octagon on $A$ Given Line $A B$.- Extend line $A B$ in both directions. Erect perpendiculars at $A$ and $B$. With centers $A$ and $B$ and radius $A B$ describe the semicircle $C E B$ and $A F_{2}$. Bisect the quadrants $C E$ and $D F$ in $\mathrm{I}$ and 2 , then $A \mathrm{I}$ and $B 2$ will be two more sides of the octagon. At $\mathrm{I}$ and 2 erect perpendiculars I, 3 and 2, 4 equal to $A B$. Draw I-2 and 3-4. Make the 
perpendiculars at $A$ and $B$ equal to $1-2$ or 3-4-viz., $A 5$ and $B 6$. Complete the octagon by drawing $3-5,5-6$, and 6-4.

Fig. 48. To Draw a Right Line Equal to Half the Circumference of a Given Circle.-Draw a diameter $A B$. Draw line $A C$ perpendicular to $A B$ and equal to three times the radius of the circle. Draw another perpendicular at $B$ to $A B$. With center $B$ and radius of the circle cut off arc $B D$, bisect it and draw a line from the center of the circle through the bisection, cutting line $B$ in $E$. Join $E C$. Line $E C$ will be equal to half the circumference of circle $A$.

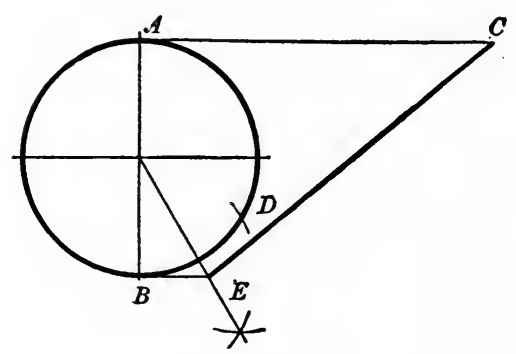

FIG. 48.

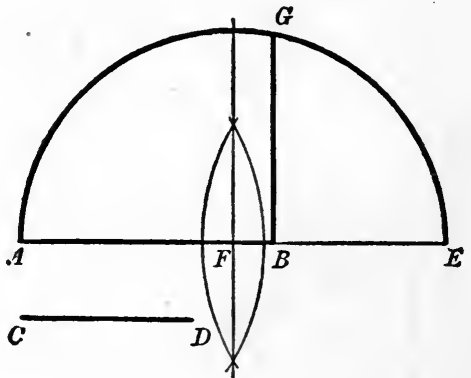

FIG. 49.

Fig. 49. To Find a Mean Proportional to two Given Right Lines.-Extend the line $A B$ to $E$ making $B E$ equal to $C D$. Bisect $A E$ in $F$. From $F$ with radius $F A$ describe a semicircle. At $B$ where the two given lines are joined erect a perpendicular to $A E$ cutting the semicircle in $G$. $B G$ will be a mean proportional to $C D$ and $A B$.

Fig. 50. To Find a Third Proportional (less) to Two Given Right Lines $A B$ and $C D$.-Make $E F=$ the given line $A B$. Draw $E G$ making an angle with $E F=D C$. Join $F G$. From $E$ with $E G$ as radius cut $E F$ in $H$. Draw 
$H$ parallel to $F G$, cutting $E G$ in $I . \quad E I$ is the third proportional (less) to the two given lines.

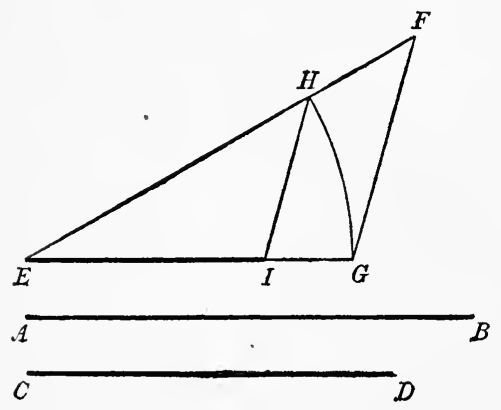

FIG. 50.

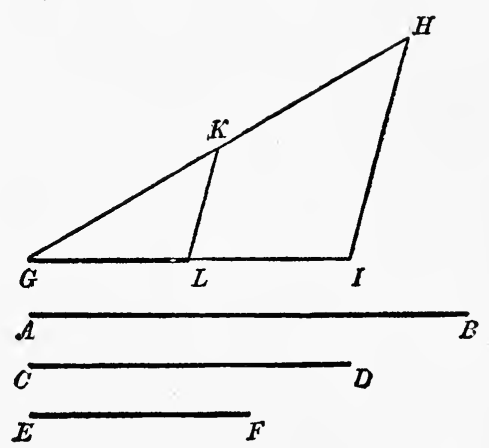

FIG. 5 I.

Fig. 5 I. To Find a Fourth Proportional to three Given Right Lines $A B, C D$, and $E F$.-Make $G H=$ the given line $A B$. Draw $G I=C D$, making any convenient angle to $G H$. Join $H I$. From $G$ lay off $G H=E F$. From $K$ draw a parallel to $H I$ cutting $G I$ in $L . G L$ is the fourth proportional required.

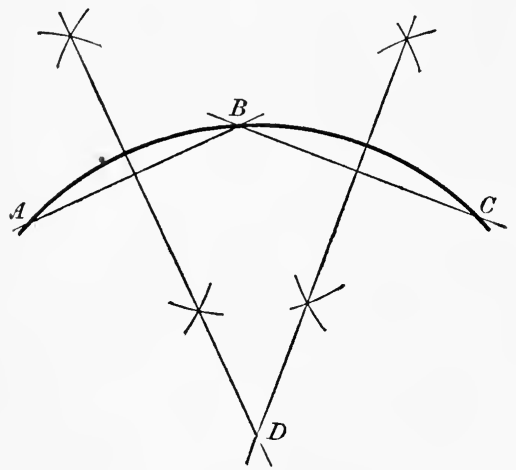

FIG. 52.

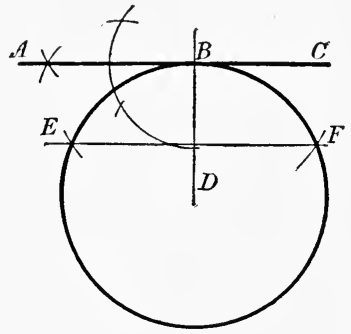

FIG. 53 .

Fig. 52. To Find the Center of a Given Arc $A B C$. -Draw the chords $A B$ and $C D$ and bisect them. Extend the bisection lines to intersect in $D$ the center required. 
FIG. 53. TO DRAW A LINE TANGENT TO AN ARC OF A CIRCLE. (Ist.) When the center is not accessible. Let $B$. be the point through which the tangent is to be drawn. From $B$ lay off equal distances as $B E, B F$. Join $E F$ and through $B$ draw $A B C$ parallel to $E F$. (2d.) When the center $D$ is given. Draw $B D$ and through $B$ draw $A B C$ perpendicular to $B D . A B C$ is tangent to the circle at the point $B$

Fig. 54. To Draiv Tangents to the Circle $C$ from The Point $A$ without It.-Draw $\backslash A C$ and bisect it in $E$. From $E$ with radius $E C$ describe an arc cutting circle $C$ in $B$ and $D$. Join $C B, C D$. Draw $A B$ and $A D$ tangent to the circle $C$.

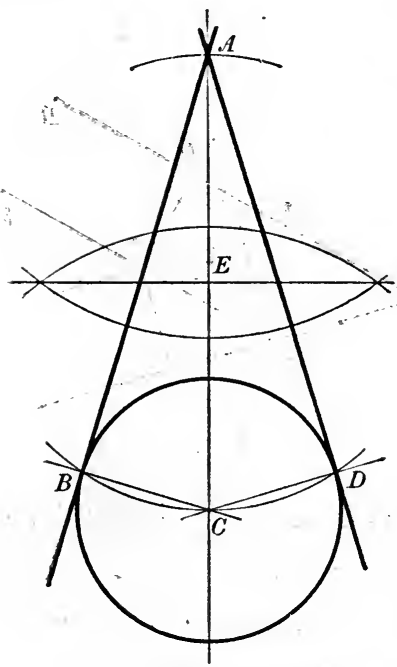

FIG. 54.

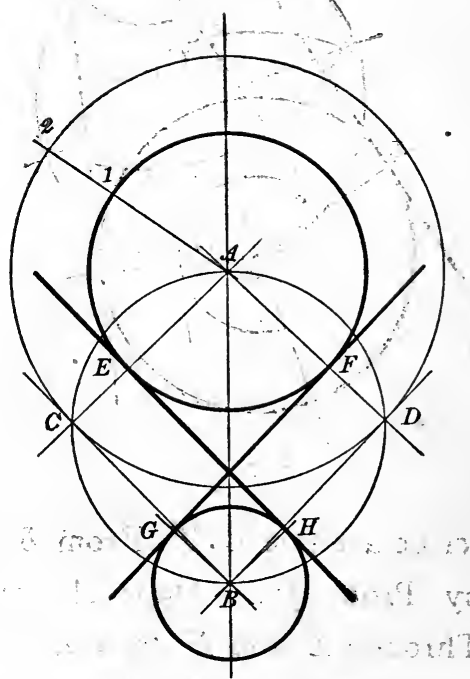

FIG: 55 .

Fig. 55. To Draw a Tangent Between two CirCLES.-Join the centers $A$ and $B$. Draw any radial line from $A$ as $A 2$ and make $\mathrm{I}-2=$ the radius of circle $B$. From $A$ with radius $A-2$ describe a circle $C_{2} D$. From center $B$ 
draw tangents $B C$ and $B D$ to circle $C_{2} D$ at the points $C$ and $D$ by preceding problem. Join $A C$ and $A D$ and through the points $E$ and $F$ draw parallels $F G$ and $E H$ to $B D$ and $B C$. $F G$ and $E H$ are the tangents required.

Fig. 56. To Draw Tangents to two Given CirCLES $A$ AND $B$.- Join $A$ and $B$. From $A$ with a radius equal to the difference of the radii of the given circles de-

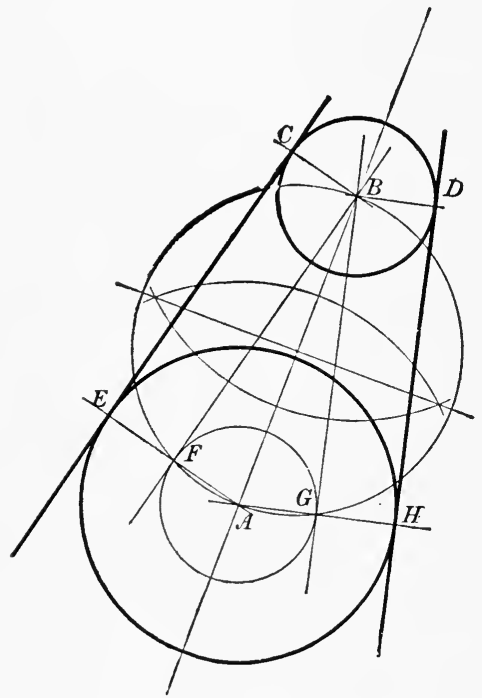

FIG. 56.

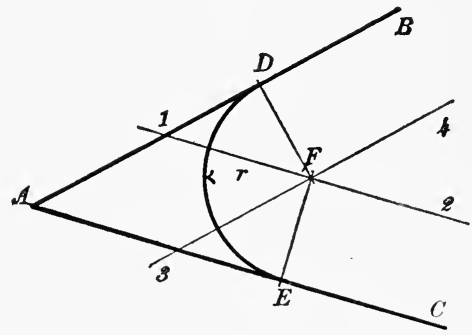

FIG. 57 .

scribe a circle $G F$. From $B$ draw the tangents $B F$ and $B G$, by Prob. 37. Draw $A F$ and $A G$ extended to $E$ and $H$. Through $E$ and $H$ draw $E C$ and $H D$ parallel to $B F$ and $B G$ respectively. $E C$ and $D H$ are the tangents required.

Fig. 57. TO Draw an Arc of a Circle of Given Radius Tangent to two Straight Lines. $-A B$ and $A C$ are the two straight lines, and $r$ the given radius. At a distance $=r$ draw parallels $\mathbf{I}-2$ and $3-4$ to $A C$ and $A B$, inter- 
secting at $F$. From $F$ draw perpendiculars $F D$ and $F E$. With $F$ as center and $F D$ or $F E$ as radius describe the required arc, which will be tangent to the two straight lines at the points $D$ and $E$.

Fig. 58. To Draw an Arc of a Circle Tangent to two Straight Lines $B C$ and $C D$ when the MidPOSITION $G$ Is GIVEN.-Draw $C A$ the bisection of the angle $B C D$ and $E F$ at right angles to it through the given point $G$. Next bisect either of the angles $F E B$ or $E F D$. The bisection line will intersect the central line $C A$ at $A$, which will be the center of the arc. From $A$ draw perpendiculars $A \mathrm{I}$ and $A 2$, and with either as a radius and $A$ as center describe an arc which will be tangent to the lines $B C$ and $C D$ at the points I and 2.

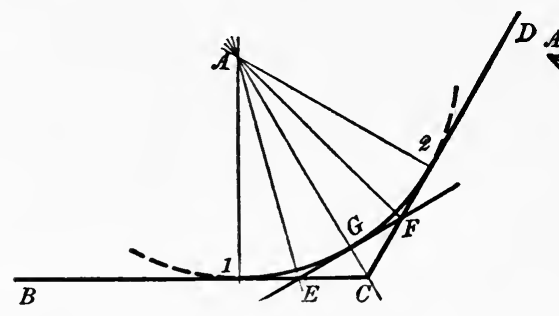

Fig. 58.

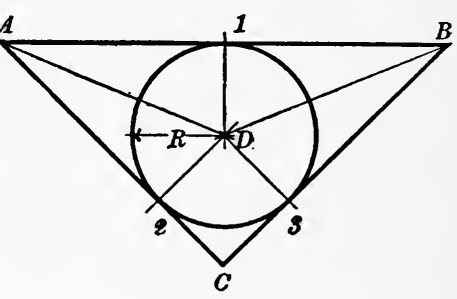

FIG. 59.

Fig. 59. To Inscribe a Circle within a Triangle $A B C$.-Bisect the angles $A$ and $B$. The bisectors will meet in $D$. Draw $D$ I perpendicular to $A B$. Then with center $D$ and radius $=D_{1}$ describe a circle which will be tangent to the given triangle at the points I, 2, 3 .

Fig. 6o. To Draw an Arc of a Circle of Given Radius $R$ tangent to two Given Circles $A$ and $B .-$ From $A$ and $B$ draw any radial lines as $A_{3}, B_{4}$. Outside the circumference of each circle cut off distances $I-3$ and $2-4$ 
each $=$ the given radius $R$. Then with center $A$ and radius $A-3$, and center $B$ and radius $B-4$ describe arcs intersecting at $C$. Draw $C A, C B$ cutting the circles at 5 and 6 . With centre $C$ and radius $C_{5}$ or $C 6$ describe an arc which will be tangent at points 5 and 6 .

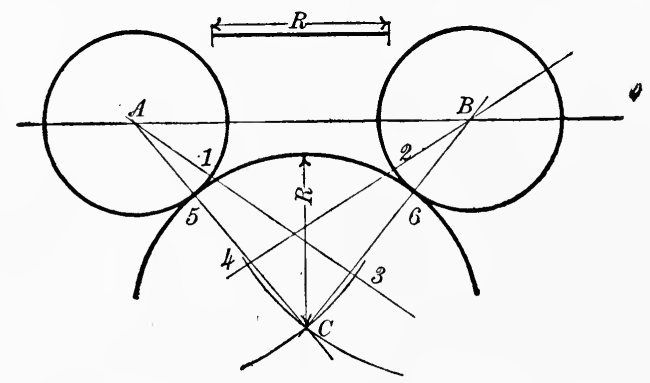

FIG. 60.

Fig. 6i. To Draw an Arc of a Circle of Given Radius $R$ tangent to two Given Circles $A$ and $B$

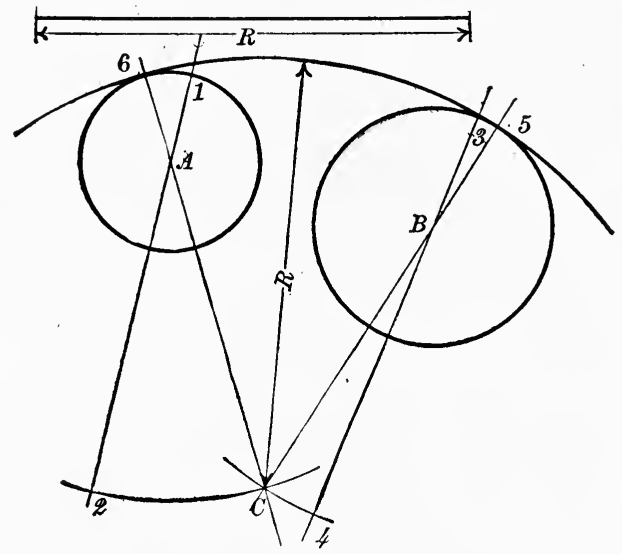

FIG. 6r.

when the Arc includes the Circles.-Through $A$ and $B$ draw convenient diameters and extend them indefinitely. On 
these measure off the distances $1-2$ and $3-4$, each equal in length to the given radius $R$. Then with center $A$ and radius $A 2$, center $B$ and radius $B 4$, describe arcs cutting at $C$. From $C$ draw $C_{5}$ and $C 6$ through $B$ and $A$. With center $C$ and radius $C 6$ or $C_{5}$ describe the arc 6,5 , which will be tangent to the circles at the points 6 and 5 .

Fig. 62. To Draw an ARC of a Circle of -Given Radius $R$ tangent to Two Givén Circles $A$ and $B$ WheN the ARC INCLudes ONe CirCle AND EXCLUDES THE. OTHER.-Through $A$ draw any diameter and make $\mathrm{I}-2=R$.

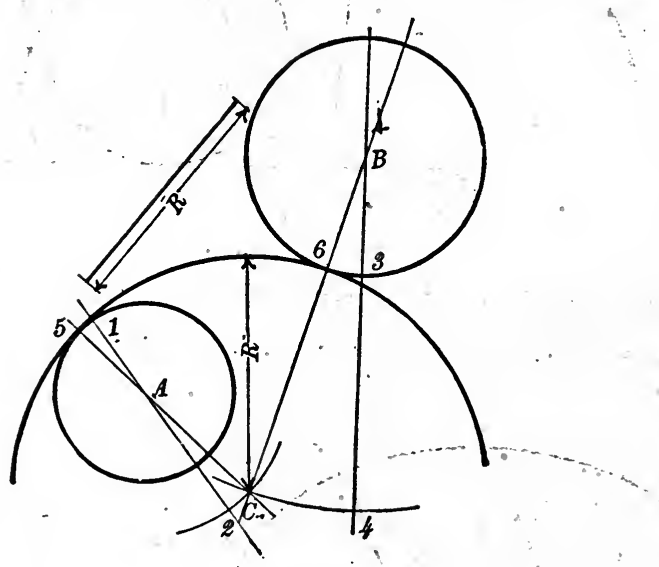

Fíg. 62.

From $B$ draw any radius and extend it, making 3-4 $=R$. With center $A$ and radius $A 2$ and center $B$ and radius $B_{4}$ describe arcs cutting at $C$. With $C$ as center and radius $=C_{5}$ or $C 6$ describe the arc $5,6$.

Fig. 63. Draw an Arc of a Circle of Given Radius $R$ tangent to a Straight Line $A B$ and a Circle $C D$.-From $E$, the center of the given circle, draw an arc of a 
circle 1,2 concentric with $C D$ at a distance $R$ from it, and also a straight line 3,4 parallel to $A B$ at the same distance $R$ from $A B$. Draw $E O$ intersecting $C D$ at 5. Draw the perpendicular 06 . With center $O$ and radius 06 or $O_{5}$ describe the required arc.

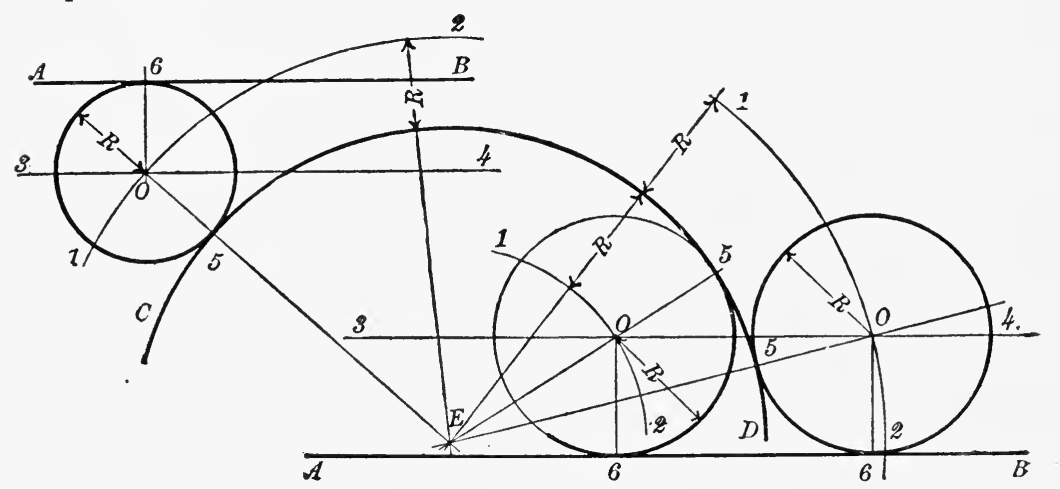

FiG. 63.

Fig. 64. To Describe AN Ellipse Approximately BY MEANS OF THREE RADII (F. R. Honey's method).-

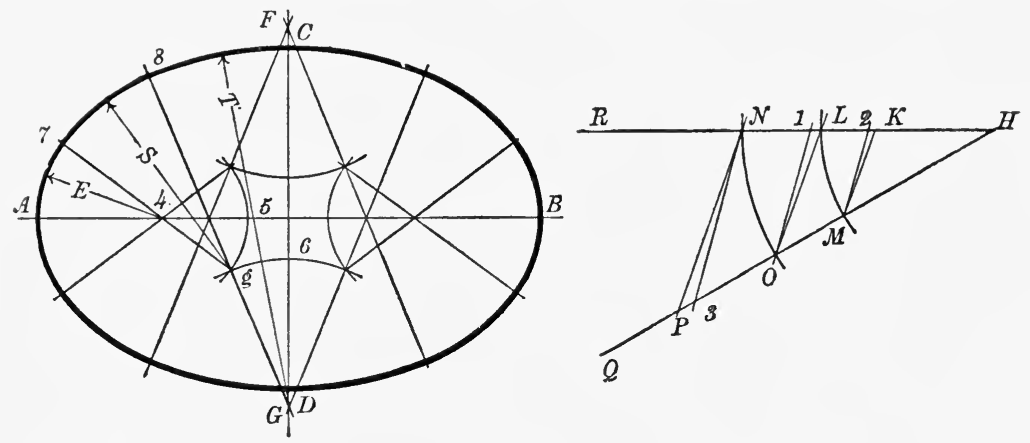

FIG. 64 .

Draw straight lines $R H$ and $H Q$, making any convenient angle at $H$. With center $H$ and radii equal to the semi-minor and. 
semi-major axes respectively, describe arcs $L M$ and $N O$. Join $L O$ and draw $M K$ and $N P$ parallel to $L O$. Lay off $L_{\mathrm{I}}=\frac{1}{8}$ of $L N$. Join $O$ I and draw $M_{2}$ and $N_{3}$ parallel to $O$ I. Take $\mathrm{H}_{3}$ for the longest radius $(=\mathrm{T}), \mathrm{H}_{2}$ for the shortest radius $(=E)$, and one-half the sum of the semi-axes for the third radius $(=S)$, and use these radii to describe the ellipse as follows: Let $A B$ and $C D$ be the major and minor axes. Lay off $A_{4}=E$ and $A_{5}=S$. Then lay off $C G=T$ and $C 6=S$. With $G$ as center and $G 6$ as radius draw the arc $6, g$. With center 4 and radius 4,5 , draw arc $5, g$, intersecting $6, g$ at $g$. Draw the line $G g$ and produce it making $G 8=T$. Draw $g$, 4 and extend it to 7 making $g, 7=S$. With center $G$ and radius $G C(=T)$ draw the arc $C 8$. With center $g$ and radius $g, 8(=S)$ draw the arc 8,7 . With center 4 and radius 4,7 $(=E)$ draw arc $7 A$. The remaining quadrants can be drawn in the same way.

Fig. 65. To DraW ax Ellipse having given the Axes $A B$ AND $C D$. - Draw $A B$ and $C D$ at right angles to and bisecting each other at $E$. With center $C$ and radius $E A$ cut $A B$ in $F$ and $F^{\prime}$ the foci. Divide $E F$ or $E F^{\prime}$ into a number of parts as shown at I, $2,3,4$, etc. Then with $F$ and $F^{\prime}$ as cen-

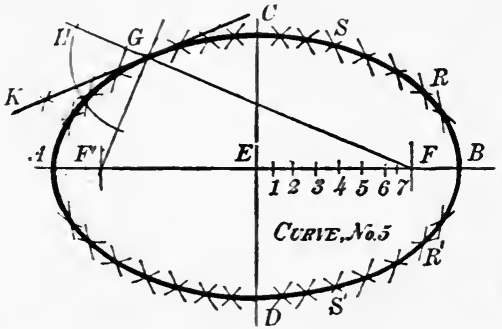

FIG. 65 .

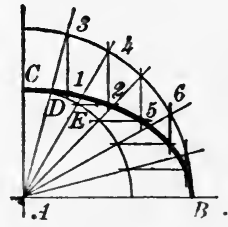

FIG. 66 .

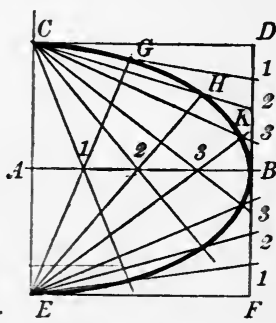

FIG. 67.

ters and $A \mathrm{I}$ and $B \mathrm{I}$, and $A 2$ and $B 2$, etc., as radii describe arcs intersecting in $R, S$, etc., until a sufficient number of points 
are found to draw the elliptic curve accurately throughout. (No. 5 of the "Sibley College Set" of irregular curves is very useful in drawing this curve.) To draw a tangent to the ellipse at the point $G$ : Extend $F G$ and draw the bisector of the angle $H G F^{\prime} . \quad K G$ is the tangent required.

Fig. 66. Another Method.-Let $A B$ and $A C$ be the semi axes. With $A$ as center and radii $A B$ and $A C$ describe circles. Draw any radii as $A 3$ and $A 4$, etc. Make 3 I, 42 , etc., perpendicular to $A B$, and $D_{2}, E_{5}$, etc., parallel to $A B$. Then I, 2, 5, etc., are points on the curve.

Fig. 67. Another Method.-Place the diameters as before, and construct the rectangle $C D E F$. Divide $A B$ and $D B$ and $B F$ into the same number of equal parts as $1,2,3$ and $B$. Draw from $C$ through points $\mathrm{I}, 2,3$ on $A B$ and $B D$ lines to meet others drawn from $E$ through points I, 2, 3 on $A B$ and $F B$ intersecting in points $G H K$. GHK are points on the curve.

Fig. 68. Another Method.-Place the diameters $A B$ and $C D$ as shown in Drawing No. I. Draw any convenient
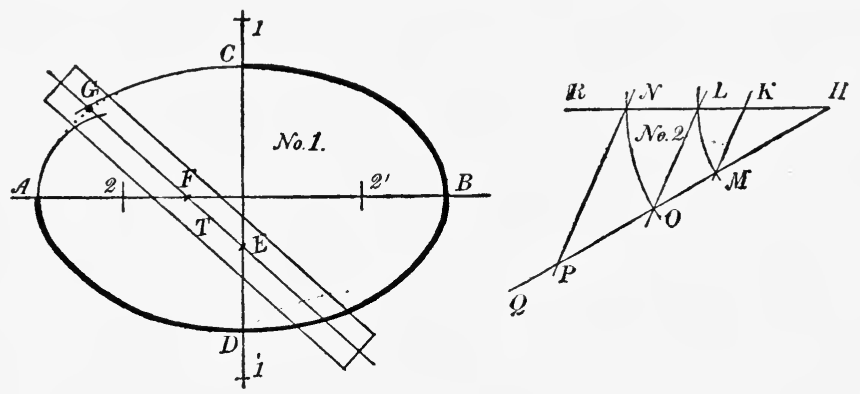

FIG. 68.

angle $R H Q$, Drawing No. 2. With center $H$ and radii equal to the semi-minor and semi-major axes describe arcs $L M I$ and 
NO. Join $L O$ and draw $M K$ and $N P$ parallel to $L O$. Then from $C$ and $D$ with a distance $=H P$ lay off the points $\mathbf{I} \mathbf{I}^{\prime}$ on the minor axis and from $A$ and $B$ with a distance $=H K$ lay off the points $22^{\prime}$. on the major axis. With centers $\mathrm{I}, \mathrm{I}^{\prime}, 2$ and $2^{\prime}$ and radii $\mathrm{I}-D$ and $2-B$, respectively, draw arcs of circles. On a piece of transparent celluloid $T$ lay off from the point $G$, $G F$ and $G E=$ the semi-minor and semi-major axes respectively. Place the point $F$ on the major axis and the point $E$ on the minor axis. If the strip of celluloid is now moved over the figure, so that the point $E$ is always in contact with the semi-minor axis and the point $F$ with the semi major axis, the necessary number of points may be marked through a small hole in the celluloid at $G$ with a sharp conical-pointed pencil, and thus complete the curve of the ellipse between the arcs of circles.

Fig. 69. To Construct a Parabola, the Base $C D$ ANd the AbScissa $A B$ BeIng given.-Draw $E F$ through $A$ parallel to $C D$ and $C E$ and $D F$ parallel to $A B$. Divide $A E$, $A F, E C$, and $F D$ into the same number of equal parts. Through the points I, 2, 3 on $A F$ and $A E$ draw lines parallel to $A B$, and through $A$ draw lines to the points 1, 2, 3 on $F D$ and $E C$ intersecting the parallel lines in points $4,5,6$, etc., of the curve.

Fig. 70. Given the Directrix $B D$ and the Focus $C$ to Draw a Parabola and a Tangent to It at the Point 3.-The parabola is a curve such that every point in the curve is equally distant from the directrix $B D$ and the focus $C$. The vertix $E$ is equally distant from the directrix and the focus, i.e. $C E$ is $=E B$. Any line parallel to the axis is a diameter. A straight line drawn across the figure at right angles to the 
axis is a double ordinate, and either half of it is an ordinate. The distance from $C$ to any point upon the curve, as 2 is always equal to the horizontal distance from that point to the directrix. Thus $C_{\mathrm{I}}=\mathrm{I}, \mathrm{I}^{\prime}, C_{2}$ to $2,2^{\prime}$, etc. Through $C$ draw $A C F$ at right angles to $B D, A C F$ is the axis of the

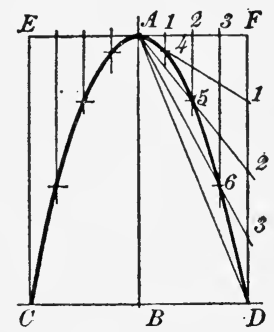

FIG. 69.

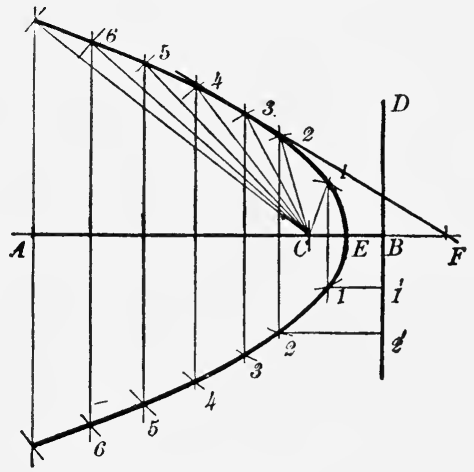

FIG. 70.

curve. Draw parallels to $B D$ through any points in $A B$, and with center $C$ and radii equal to the horizontal distances of these parallels from $B D$ describe arcs cutting in the points $I$, $2,3,4$, etc. These are points in the curve. The tangent to the curve at the point 3 may be drawn as follows: Produce $A B$ to $F$. Make $E F=$ the horizontal distance of ordinate 33 from $E$. Draw the tangent through $3 F$.

Fig. 7I. TO Draw AN Hyperbola, Having Given the Diameter $A B$, the Abscissa $B D$, and Double OrdiNATE $E F$. - Make $F_{4}$ parallel and equal to $B D$. Divide $D F$ and $F_{4}$ into the same number of equal parts. From $B$ draw lines to the points in ${ }_{4} F$ and from $A$ draw lines to the points in $D F$. Draw the curve through the points where the lines correspondingly numbered intersect each other. 
Fig. 72. To Construct an Oval the Width $A B$ BEING Given.-Bisect $A B$ by the line $C D$ in the point $E$, and with $E$ as center and radius $E A$ draw a circle cutting $C D$ in

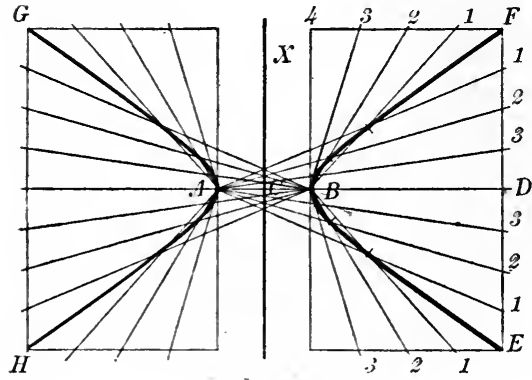

Fig. 7r.

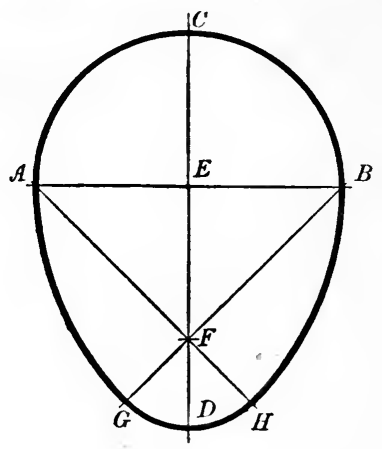

FIG. 72.

$F$. From $A$ and $B$ draw lines through $F$. From $A$ and $B$ with radius equal to $A B$ draw arcs cutting the last two lines in $G$ and $H$. From $F$ with radius $F G$ describe the arc $G H$ to meet the arcs $A G$ and $B H$, which will complete the oval.

Fig. 73. Given an Ellipse to Find the Axes and Foci.-Draw two parallel chords $A B$ and $C D$. Bisect each of these in $E$ and $F$. Draw $E F$ touching the ellipse in I and 2. This line divides the ellipse obliquely into equal parts. Bisect $\mathbf{I}, 2$ in $G$, which will be the center of the ellipse. From $G$ with any radius draw a circle cutting the ellipse in $H / J K$. Join these four points and a rectangle will be formed in the ellipse. Lines $L . M$ and $N O$, bisecting the sides of the rectangle, will be the diameters or axes of the ellipse. With $N$ or $O$ as centers and radius $=G L$ the semi-major axis, describe arcs cutting the major axis in $P$ and $Q$ the foci.

Fig. 74. To Construct a Sipral of one RevoluTION.-Describe a circle using the widest limit of the spiral as 
a radius. Divide the circle into any number of equal parts as $A, B, C$, etc. Divide the radius into the same number of equal parts as I to I2. From the center with radius I2, I describe an arc cutting the radial line $B$ in $\mathrm{I}^{\prime}$. From the center continue to draw arcs from points 2,3 , 4, etc., cutting the corresponding radii $C, D, E$, etc. in the points $2^{\prime}, 3^{\prime}, 4^{\prime}$, etc. From I 2 trace the Archimedes Spiral of one revolution.

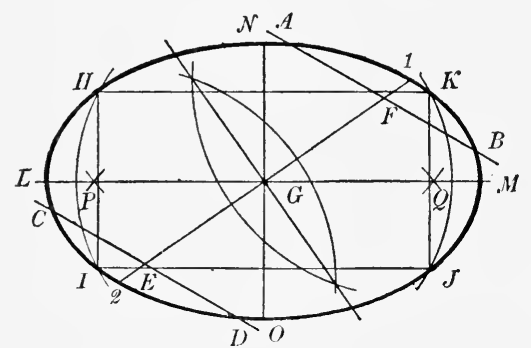

FIG. 73 .

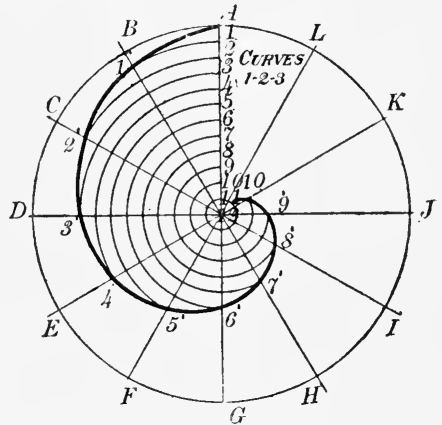

FIG. 74 .

Fig. 75. To Describe a Spiral of any Number of Revolutions, E.G., 2.-Divide the circle into any number of equal parts as $A, B, C$, etc., and draw radii. Divide the radius $A 12$ into a number of equal parts corresponding with the required number of revolutions and divide these into the same number of equal parts as there are radii, viz., I to I2. It will be evident that the figure consists of two separate spirals, one from the center of the circle to 12 , and one from 12 to $A$. Commence as in the last problem, draw. ing arcs from I, 2, 3, etc., to the correspondingly numberea radii, thus obtaining the points marked $I^{\prime}, 2^{\prime}, 3^{\prime}$, ctc. The first revolution completed, proceed in the same manner to find the points $1^{\prime \prime}, 2^{\prime \prime}, 3^{\prime \prime}$, etc. Through these points trace the spiral of two revolutions. 
Fig. 76. To Construct the Involute of the CiRCLE O.-Divide the circle into any number of equal parts and draw radii. Draw tangents at right angles to these radii. On the tangent to radius I lay off a distance equal to one of the parts into which the circle is divided, and on each of

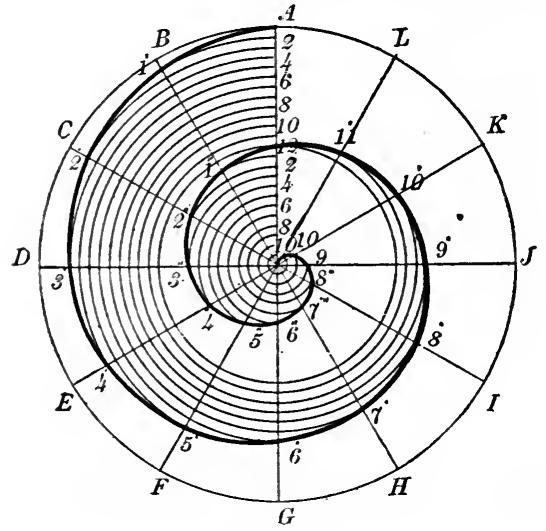

FIG. 75 .

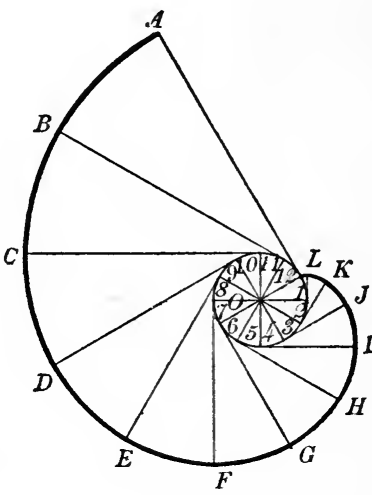

Fig. 76 .

the tangents set off the number of parts corresponding to the number of the radii. Tangent 12 will then be the circumference of the circle unrolled, and the curve drawn through the extremities of the other tangents will be the involute.

Fig. 77. To Describe an Ionic Volute.-Divide the given height into seven equal parts, and through the point 3 the upper extremity of the third division draw 3,3 perpendicular to $A B$. From any convenient point on 33 as a center, with radius equal to one-half of one of the divisions on $A B$, describe the eye of the volute $N P N M$, shown enlarged at Drawing No. 2. $N N$ corresponds to line 3, 3, Drawing No. I. Make $P M$ perpendicular to $N N$ and inscribe the square $N P N M$, bisect its sides and draw the square II, I2, 
I3, I4. Draw the diagonals II, I 3 and I2, I4 and divide them as shown in Drawing No. 2. At the intersections of the horizontal with the perpendicular full lines locate the points I, 2, 3, 4, etc., which will be the centers of the quadrants of the outer curve. The centers for the inner curve will be found at the intersections of the horizontal and per-
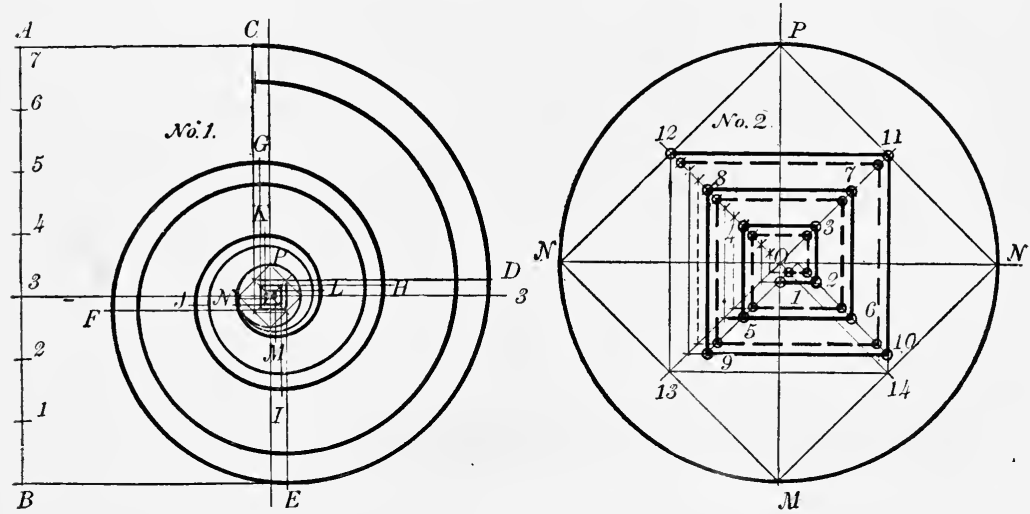

FIG. 77 .

pendicular broken lines, drawn through the divisions on the diagonals. Then with center $\mathrm{I}$ and radius $\mathrm{I} P$ draw $\operatorname{arc} P N$, and with center 2 and radius $2 N$ draw arc $N M$, with center 3 and radius $3 M$ draw arc $M L$, etc. The inner curve is drawn in a similar way, by using the points on the diagonals indicated by the broken lines as centers.

Fig. 78. To De-Cribe the Cycloid- $-A B$ is the director, $C B$ the generating circle, $X$ a piece of thin transparent celluloid, with one side dull on which to draw the circle $C$. At any point on the circle $C$ puncture a small hole with a sharp needle, and place the point $C$ tangent to the director $A B$ at the point from which the curve is to be drawn. Hold the celluloid at this point with a needle, and rotate it until 
the arc of the circle $C$ intersects the director $A B$. Through the point of intersection stick another needle and rotate $X$ until the circle is again tangent to $A B$, and through the puncture at $C$ with a $4 \mathrm{H}$ pencil, sharpened to a fine conical point, mark the first point on the curve. So proceed until sufficient points have been found to complete the curve.

(NOTE.-The thin celluloid was first used as a drawing instrument by Professor H. D. Williams, of Sibley College, Cornell University.)

Fig. 79. To Find the Length of a Given Arc of a Circle approximately. - Let $B C$ be the given arc. Draw its chord and produce it to $A$, making $B A$ equal half the

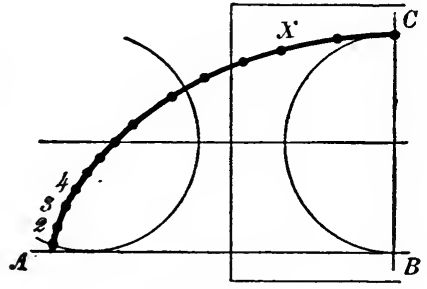

Fig. 78.

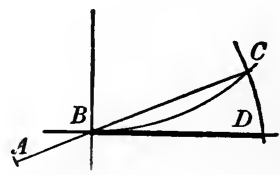

Fis. 79.

chord. With center $A$ and radius $A C$ describe arc $C D$ cutting the tangent line $B D$ at $D$, and making it equal to the $\operatorname{arc} B C$.

Fig. 8o. To Describe the Cycloid by the Old METHOD - Divide the director and the generating circle into the same number of equal parts. Through the center $a$ draw $a g$ parallel to $A B$ for the line of centers, and divide it as $A B$ in the points $b, c, d, e, f$, and $g$. With centers $f, e, d$, etc., describe arcs tangent to $A B$, and through the points of division on the generating circle $\mathrm{I}, 2,3$, etc., draw lines parallel to 
$A B$ cutting the arcs in the points $\mathrm{I}^{\prime}, \mathbf{2}^{\prime}, 3^{\prime}$, etc. These will be points in the curve.

An approximate curve may be drawn by arcs of circles. Thus, taking $f^{\prime}$ as center and $f^{\prime} g^{\prime}$ as radius, draw $\operatorname{arc} g^{\prime} I^{\prime}$.

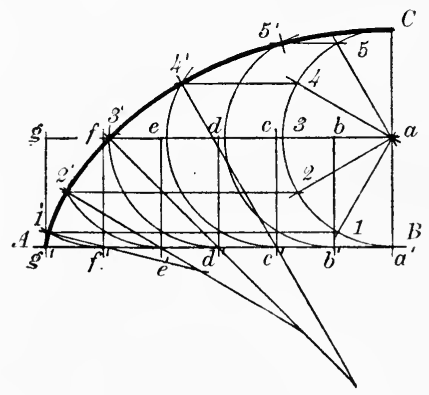

FIG. 8o.

Produce $\mathrm{I}^{\prime} f^{\prime}$ and $2^{\prime} c^{\prime}$ until they meet at the center of the second arc $2^{\prime} f^{\prime}$, etc.

Fig. 8i. To Describe the Epicycloti ani the HYPOCYCLOID. - Divide the generating circle into any number of equal parts, I, 2, 3, etc., and set off these lengths from $C$ on the directing circle $C B$ as $c^{\prime}, d^{\prime}, c^{\prime}$, etc. From $A$ the center of the directing circle draw lines through $c^{\prime}, d^{\prime}, c^{\prime}$, etc., cutting the circles of centers in $c, d, c$, etc. From each of these points as centers describe arcs tangent to the directing circle. From center $A$ draw arcs through the points of division on the generating circle, cutting the arcs of the generating circles in their several positions at the points $I^{\prime}, 2^{\prime}, 3^{\prime}$, etc. These will be points in the curve.

Fici. 82. ANother Method.-Draw the generating circle on the celluloid and roll it on the outside of the generating circle $B C$ for the Epicycloid, and on the inside for the 
Hypocycloid, marking the points in the curve $\mathrm{I}, 2,3$, etc., in similar manner to that described for the Cycloid.

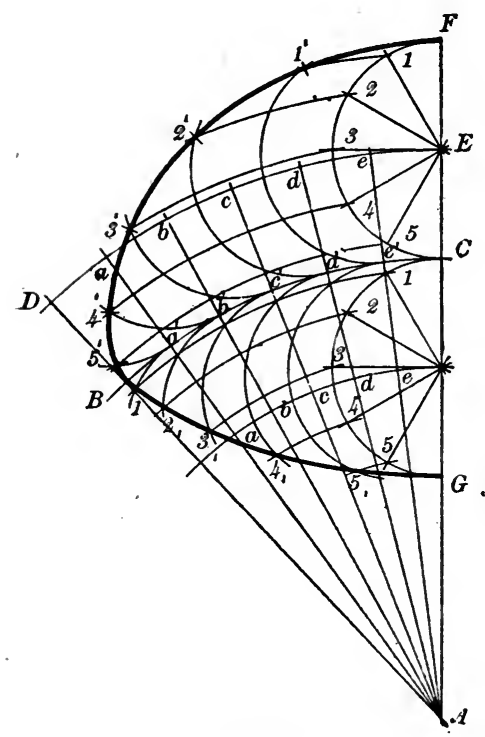

FIG. 8I.
FIG. 82.

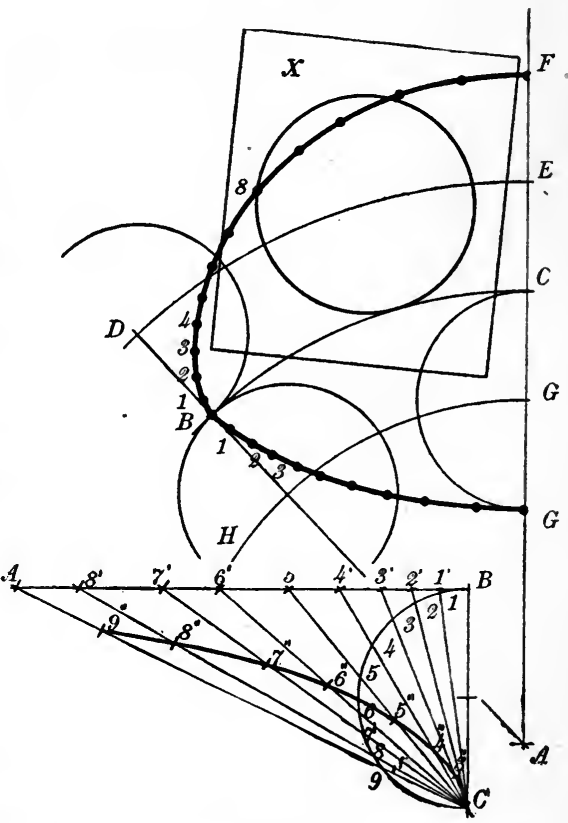

FIG. 83.

Fig. 83. To Draw the Cissold.-Draw any line $A B$ and $B C$ perpendicular to it. On $B C$ describe a circle. From the extremity $C$ of the diameter draw any number of lines, at any distance apart, passing through the circle and meeting the line $A B$ in $\mathrm{I}^{\prime}, 2^{\prime}, 3^{\prime}$, etc. Take the length from $A$ to 9 and set it off from $C$ on the same line to $9^{\prime \prime}$. Take the distance from $8^{\prime}$ to 8 and set it off from $C$ on the same line to $8^{\prime \prime}$, etc., for the other divisions, and through $9^{\prime \prime}, 8^{\prime \prime}, 7^{\prime \prime}, 6^{\prime \prime}$, etc., draw the curve. 
Fig. 84. To Draw Schiele's Anti-friction Curve. -Let $A B$ be the radius of the shaft and $B \mathrm{I}, 2,3,4$, etc., its axis. Set off the radius $A B$ on the straight edge of a piece of stiff paper or thin celluloid and placing the point $B$ on the division $\mathrm{I}$ of the axis, draw through point $A$ the line $A \mathrm{I}$. Then lower the straight edge until the point $B$ coincides with 2 and the point $A$ just touches the last line drawn, and draw $a 2$, and so proceed to find the points $a, b, c$, etc. Through these points draw the curve.

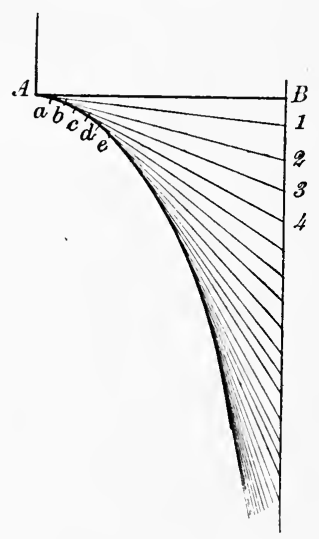

FIG. 84 .

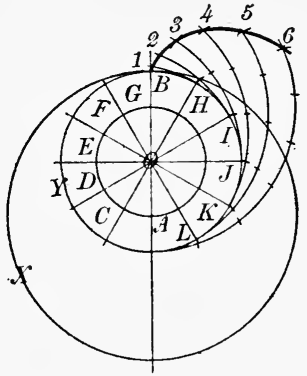

FIG. 85 .

Fig. 85. TO DEscribe AN INTERIOR EpICYClOID.Let the large circle $X$ be the generator and the small circle $Y$ the director. Divide circle $Y$ into any number of equal parts, as $B, H, I, J$, etc. Draw radial lines and make $H C$, $I D, J E, K F$, etc., each equal to the radius of the generator $X$. With centers $C, D, E$, etc., describe arcs tangent at $H, I, J$, etc. Make $H_{\mathrm{I}}$ equal to one of the divisions of the director as $B H$. Make $I 2$ equal to two divisions, $J_{3}$, three divisions, etc., and draw the curve through the points I, 2, 3, 4 . 
etc. This curve may also be described with a piece of celluloid in a similar way to that explained for the cycloid.

It may not be out of place here to describe a few of the

\section{MOULDINGS USED IN ARCHITECTURAL WORK,}

since they are often found applied to mechanical constructions.

Fig. 86. To Describe ThE "Scotia."- I, I is the top line and 4,4 the bottom line. From I drop a perpendicular I, 4; divide this into three equal parts, as I, 2, and 3 . Through the point 2 draw $a b$ parallel to $\mathrm{I}, \mathrm{I}$. With center 2 and radius $2, \mathrm{I}$ describe the semicircle $a \mathrm{I} b$, and with center $b$ and radius $b a$ describe the arc $a 5$ tangent to 4,4 at 5 , draw the fillets I, I and 4,4 .

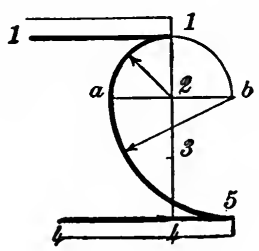

Fig. 86.

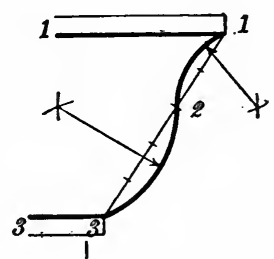

FIG. 87 .

Fig. 87. To Describe the "Cyma Recta."-Join I, 3 and divide it into five equal parts, bisect I, 2 and 2, 3, and with radius equal to I, 2 and 2,3 respectively describe arcs I, 2 and 2, 3. Draw the fillets I, I and 3,3 and complete the moulding.

Fig. 88. To Describe the "Cavetto" or "HolLOw."-Divide the perpendicular I, 2 into three equal parts and make 2, 3 equal to two of these. From centers $I$ and 3 with a radius somewhat greater than the half of $\mathrm{I}, 3$, describe arcs intersecting at the center of the arc 1,3 , 
Fig. 89. To Describe the “ Echinus,” “Quarter Round,” OR “'Ovolo." -Draw I, 2 perpendicular to 2, 3 , and divide it into three equal parts. Make 2, 3 equal to two of these parts. From the points 2 and 3 with a radius greater than half 1,3 , describe arcs cutting in the center of the required curve.

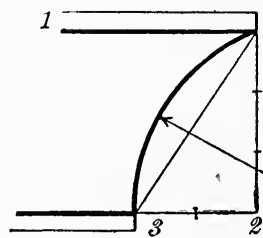

FIG. 88 .

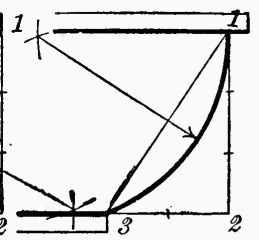

FIG 89.

Fig. 9o. To Describe The “Apophyciee."- Divide 3,4 into four equal parts and lay off five of these parts from 3 to 2 . From points 2 and 4 as centers and radius equal to 2,3 , describe arcs intersecting in the center of the curve.

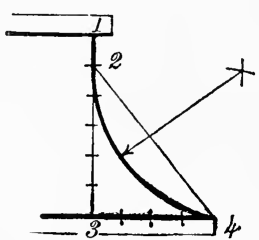

FIG. 90.

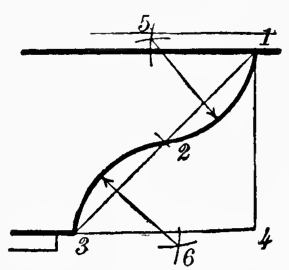

FIG. 9I.

Fig. 9i. To Describe tine "Cyma Reversa."- Make $4,3=4$, I. Join I, 3 and bisect it in the point 2 . From the points I, 2 and 3 as centers and radii equal to about two-thirds of $\mathrm{I}, 2$ draw arcs intersecting at 5 and 6 . Points 5 and 6 are the centers of the reverse curves.

Fig. 92. To Describe THe "Tokus."-Let I, 2 be the breadth. Drop the perpenficular I, 2, and bisect it in the 
point 3 . With 3 as center and radius $3, \mathbf{I}$, describe the semicircle. Draw the fillets.

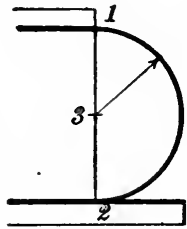

FIG. 92.

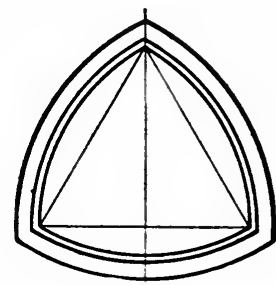

FIG. 93.

Fig. 93. An Arched Window Opening.-The curves are all arcs of circles, drawn from the three points of the equilateral triangle, as shown in the figure.

Fig. 94. To Describe the "Trefoll."-The equilateral triangle is drawn first, and the angle 1, 2, 3 bisected by the line 2, 4, which also cuts the perpendicnlar line I, 6 in the point 6 . The center of the surrounding circles 1,2 and 3 are the centers of the trefoil curves.

Fig. 95. To Describe the "Quatre Foll."-Draw the square $1,2,3,4$ in the position shown in the figure. The center of the surrounding circles, point 5 , is at the intersection of the diagonals of the square. Points I, 2, 3, 4 of the square are the centers of the small arcs.

Fig. 96. To Describe the "Cinquefoll OrnaMENT." The curves of the cinquefoil are described from the corners of a pentagon $1,2,3,4,5$. Bisect 4,5 in 6 and draw 2,6 , cutting the perpendicular in the point 7 , the center of the large circles.

Fig. 97. To DraW a Baluster.-Begin by drawing the center line, and lay off the extreme perpendicular height, 
the intermediate, perpendicular, and horizontal dimensions, and finally the curves as shown in the figure.

FIG. 94.

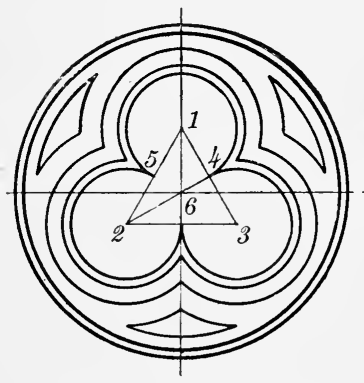

Fig. 95 .

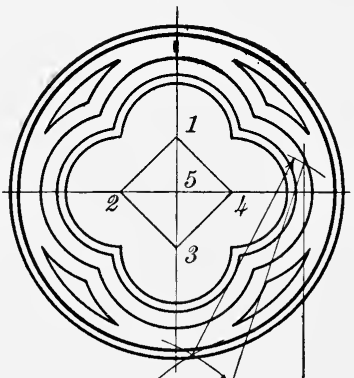

FIG. 96.

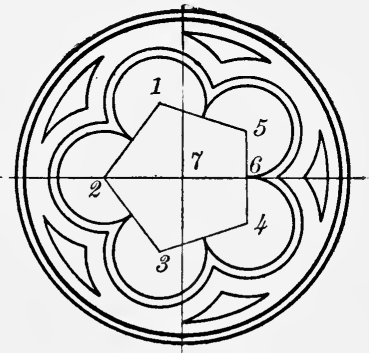

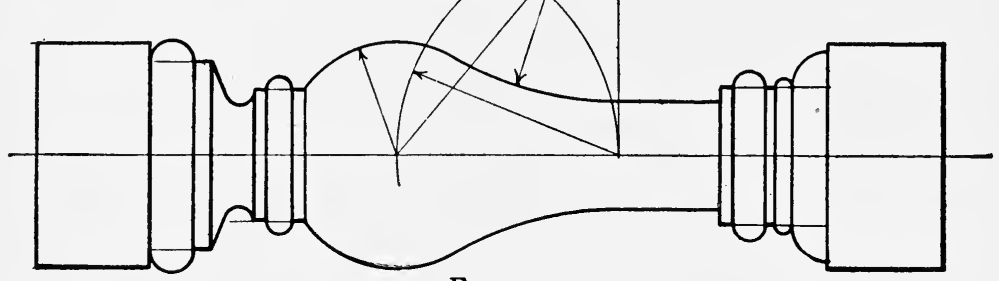

FIg. 97.

DRAWING TO SCALE.

When we speak of a drawing as having been made to scale, we mean that every part of it has been drawn proportionately and accurately, either full size, reduced or enlarged.

Very small and complicated details of machinery are usually drawn enlarged; larger details and small machines may be made full size, while larger machines and large details are shown reduced.

When a drawing of a machine is made to a reduced or enlarged scale the figures placed upon it should always give the full-size dimensions, i.e., the sizes the machine should measure when finished. 
Fig. 98. To Construct a Scale of Third Size or $4^{\prime \prime}=\mathrm{I}$ FOOT.-Draw upon a piece of tough white drawingpaper two parallel lines about " $I$ " apart and about 14 " long as shown by $a$, Fig. 98. From $A$ lay off distances equal to $4^{\prime \prime}$ and divide the first space $A B$ into 12 equal parts or inches by Prob. 12. Divide $A E$ in the same way into as many parts as it may be desired to subdivide the inch divisions on $A B$,
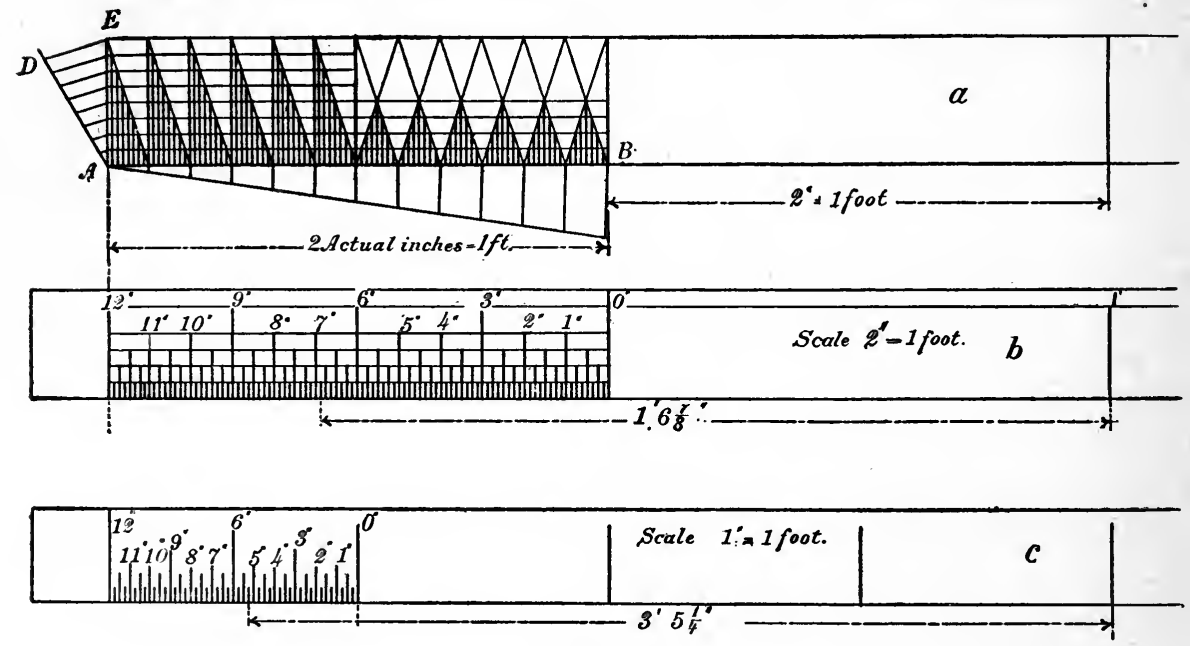

FIG. 98 .

usually 8. When the divisions and subdivisions have been carefully and lightly drawn in pencil, as shown by $a$, in Fig. 98 , then the lines denoting $\frac{1^{\prime \prime}}{8}, \frac{1^{\prime \prime}}{4}, \frac{1^{\prime \prime}}{2}, 1^{\prime \prime}$, and $3^{\prime \prime}$ should be carefully inked and numbered as shown by $(b)$. By a further subdivision a scale of $2^{\prime \prime}=1$ foot may easily be made as shown by $(c)$ in Fig. 98. 


\section{CHAPTER III.}

\section{CONVENTIONS.}

It is often unnecessary if not undesirable to represent certain things as they would actually appear in a drawing, especially when much time and labor is required to make them orthographically true.

So for economic reasons draftsmen have agreed upon conventional methods to represent many things that would otherwise entail much extra labor and expense, and serve no particular purpose.

It is very necessary, however, that all draftsmen should know how to draw the se things correctly, for occasions will often arise when such knowledge will be demanded; and besides it gives one a feeling of greater satisfaction when using conventional methods to know that he could make them artistically true if it was deemed necessary.

STANDARD CONVENTIONAL SECTION LINES.

Conventional section lines are placed on drawings to distinguish the different kinds of materials used when such drawings are to be finished in pencil, or traced for blue printing, or to be used for a reproduction of any kind.

Water-colors are nearly always used for finished drawings and sometimes for tracings and pencil drawings.

The color tints can be applied in much less time than it 
takes to hatch-line a drawing. So that the color method should be used whenever possible.

FIG. 99.-This figure shows a collection of hatch-lined sections that is now the almost universal practice among draftsmen in this and other countries, and may be considered standard.

No. I. To the right is shown a section of a wall made of rocks. When used without color, as in tracing for printing, the rocks are simply shaded with India ink and a 175 Gillott steel pen. For a colored drawing the ground work is made of gamboge or burnt umber. To the left is the conventional representation of water for tracings. For colored drawings a blended wash of Prussian blue is added.

No. 2. Convention for Marble. - When colored, the whole section is made thoroughly wet and each stone is then streaked with Payne's gray.

No. 3. Convention for Chestnut. - When colored, a ground wash of gamboge with a little crimson lake and burnt umber is used. The colors for graining should be mixed in a separate dish, burnt umber with a little Payne's gray and crimson lake added in equal quantities and made dark enough to form a sufficient contrast to the ground color.

No. 4. General Convention for Wood.-When colored the ground work should be made with a light wash of burnt sienna. The graining should be done with a writing-pen and a dark mixture of burnt sienna and a modicum of India ink.

No. 5. Convention for Black Walnut.-A mixture of Payne's gray, burnt umber and crimson lake in equal quantities is used for the ground color. The same mixture is used for graining when made dark by adding more burnt umber. 


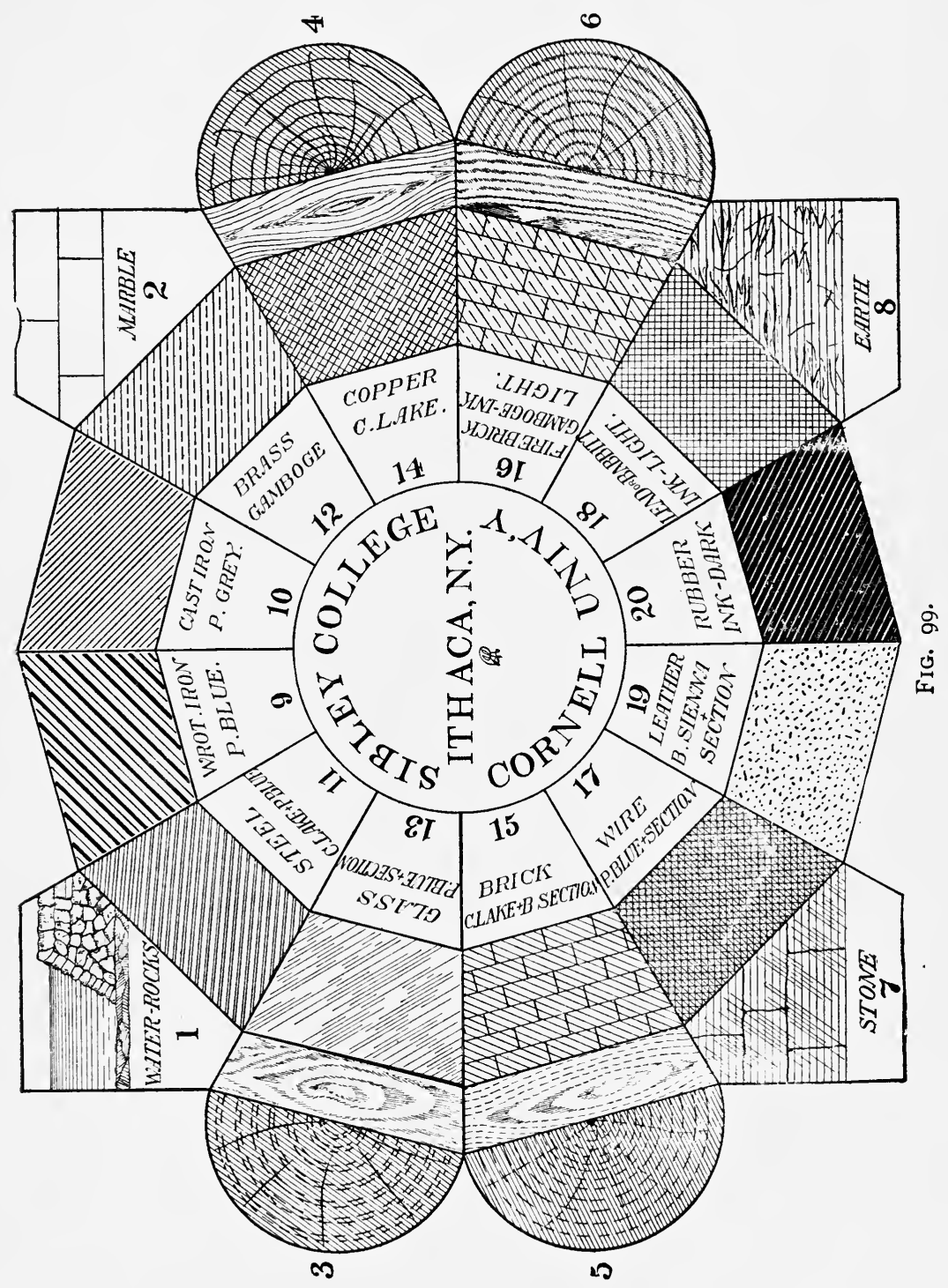


No. 6. Convention for Hard Pine.-For the ground color make a light wash of crimson lake, burnt umber, and gamboge, equal parts. For graining use a darker mixture of of crimson lake and burnt umber.

No. 7. Convention for Building-stone. - The ground color is a light wash of Payne's gray and the shade lines are added mechanically with the drawing-pen or free-hand with the writing-pen.

No. 8. Concention for Earth.-Ground color, India ink and neutral tint. The irregular lines to be added with a writing-pen and India ink.

No. 9. Section Lining for Wrought or Malleable Iron.When the drawing is to be tinted, the color used is Prussian blue.

No. I0. Cast Iron.-These section lines should be drawn equidistant, not very far apart and narrower than the body lines of the drawing. The tint is Payne's gray.

No. II. Steel.-This section is used for all kinds of steel. The lines should be of the same width as those used for castiron and the spaces between the double and single lines should be uniform. The color tint is Prussian blue with enough crimson lake added to make a warm purple.

No. 12. Brass. - This section is generally used for all kinds of composition brass, such as gun-metal, yellow metal, bronze metal, Muntz metal, etc. The width of the full lines, dash lines and spaces should all be uniform. The color tint is a light wash of gamboge.

Nos. 13-20.- The section lines and color tints for these numbers are so plainly given in the figure that further instruction would seem to be superfluous. 
CONVENTIONAL LINES.

FIG. I0O.-There are four kinds:

( I) The Hidden Line. - This line should be made of short dashes of uniform length and width, both depending somewhat on the size of the drawing. The width should always be slightly less than the body lines of the drawing, and the

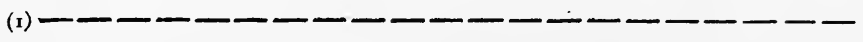

(2)

(3)

(4)

FIG. IOO.

length of the dash should never exceed $\frac{1^{\prime \prime}}{8}$. The spaces between the dashes should all be uniform, quite small, never exceeding $\frac{1}{16}{ }^{\prime \prime}$. This line is always inked in with black ink.

(2) The Line of Motion.-This line is used to indicate point paths. The dashes should be made shorter than those of the hidden line, just a trifle longer than dots. The spaces should of course be short and uniform.

(3) Center Lines.-Most drawings of machines and parts of machines are symmetrical about their center lines. When penciling a drawing these lines may be drawn continuous and as fine as possible, but on drawings for reproductions the blackinked line should be a long narrow dash and two short ones alternately. When colored inks are used the center line should be made a continuous red line and as fine as it is possible to make it.

(4) Dimcnsion Lines and Line of Section.-These lines are made in black with a fine long dash and one short dash alternately. In color they should be continuous blue lines. 
Colored lines should be used wherever feasible, because they are so quickly drawn and when made fine they give the drawing a much neater appearance than when the conventional black lines are used. Colored lines should never be broken.

\section{CONVENTIONAL BREAKS.}

FIG. IOI.-Breaks are used in drawings sometimes to indicate that the thing is actually longer than it is drawn, some-
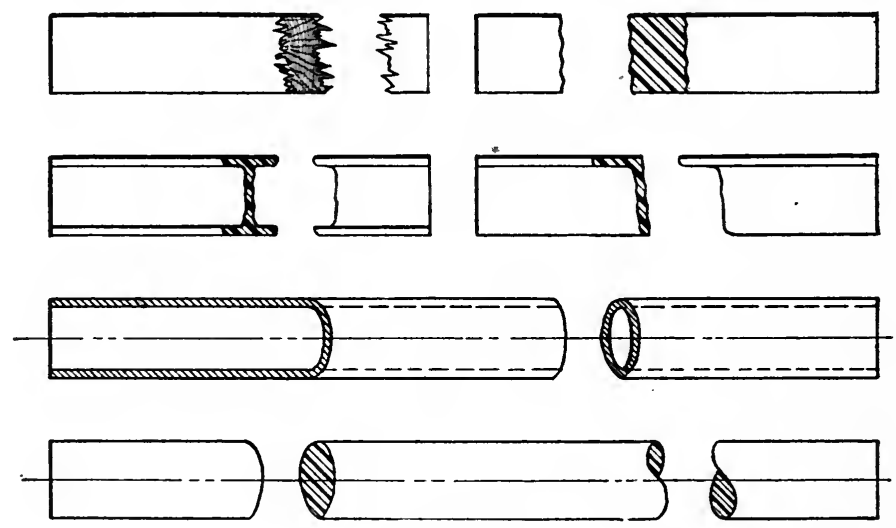

Fig. IOI.

times to show the shape of the cross-section and the kind of material. Those given in Fig. Ior show the usual practice.

\section{CROSS-SECTIONS.}

FIG. I02.-When a cross-section of a pulley, gear-wheel or other similar object is required and the cutting-plane passes through one of the spokes or arms, then only the rim and hub should be sectioned, as shown at $x x$ No. I and $z z$ No. 2 , and the arm or spoke simply outlined. Cross-sections of the arms may be made as shown at $A A$ No. 2. In working drawings of 
gear-wheels only the number of teeth included in one quadrant need be drawn; the balance is usually shown by conventional lines, e.g., the pitch line the same as a center line, viz., a long
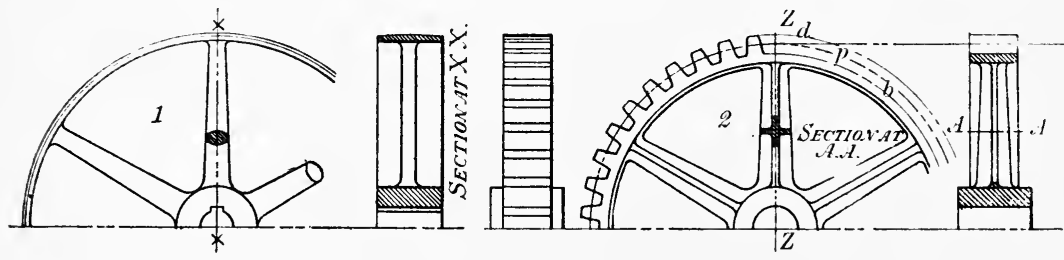

FIG. IO2.

dash and two very short ones alternately or a fine continuous red line.

The addcndum line $(d)$ and the root or bottom line $(b)$ the same as a dimension line, viz., one long dash and one short dash alternately or a fine continuous blue line. The end elevation of the gear-teeth should be made by projecting only the points of the teeth, as shown at No. 2 .

CONVENTIONAL, METHODS OF SHOWING SCREW-THREAIS IN WORKING IRAWINGS.

FIG. I03.-No. I, shows the convention for a double V thread, U. S. standard;' No. 2, a single V thread; No. 3, a single square thread; No. 4, a single left-hand $\mathrm{V}$ thread; No. 5, a double right-hand square thread; No. 6, any thread of small diameter; No. 7, any thread of very small diameter. The true methods for constructing these threads are explained on pages 99-10I, Figs. I37-1 39.

In No. 6. the short wide line is equal to the diameter of the thread at the bottom. The distance between the 
longer narrow lines is equal to the pitch, and the inclination is equal to half the pitch.

The short dash lines in No. 7 should be made to corre-
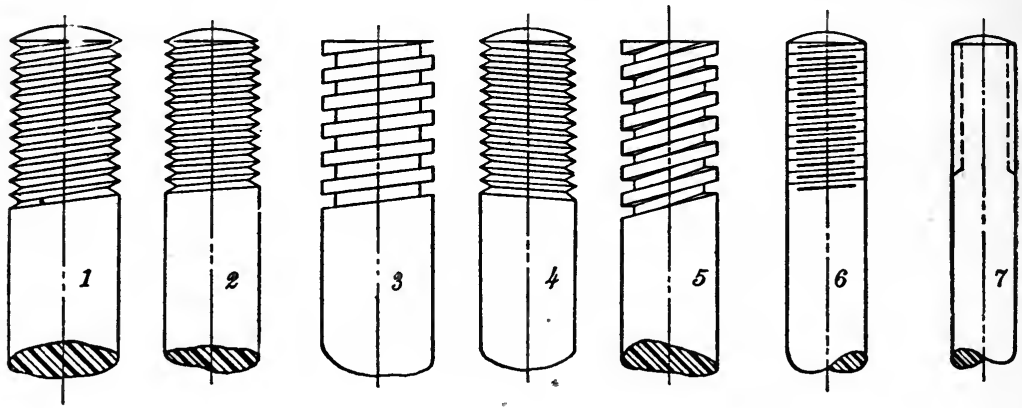

FIG. I03.

spond to the diameter of the thread at the bottom. After some practice these lines can be drawn accurately enough by the eye. 


\section{CHAPTER IV.}

\section{LETTERING AND FIGURING.}

THis subject has not been given the importance it deserves in connection with mechanical drawing. Many otherwise excellent drawings and designs as far as their general appearance is concerned have been spoiled by poor lettering and figuring.

All lettering on mechanical drawings should be plain and legible, but the letters in a title or the figures on a drawing should never be so large as to make them appear more prominent than the drawing itself.

The best form of letter for practical use is that which gives the neatest appearance with a maximum of legibility and requires the least amount of time and labor in its construction.

This would naturally suggest a "free-hand " letter, but before a letter can be constructed "free-hand" with any degree of efficiency, it will be necessary to spend considerable time in acquiring a knowledge of the form and proportions of the particular letter selected.

It is very desirable then that after the student has carefully constructed as many of the following plates of letters and numbers as time will permit and has acquired a sufficient knowledge of the form and proportions of at least the "Roman" and "Gothic" letters; he should then adopt some one 
style and practice that at every opportunity, until he has attained some proficiency in its free-hand construction.

When practicing the making of letters and numbers freehand, they should be made quite large at first so as to train the hand.

The "Roman" is the most legible letter and has the best appearance, but is also the most difficult to make well, either free-hand or mechanically. However, the methods given for its mechanical construction, Figs. 104 and 105, will materially modify the objections to its adoption for lettering mechanical drawings.

The "Gothic" letter is a favorite with mechanical draftsmen, because it is plain and neat and comparatively easy to construct. (See Fig. 106.)

Among the type specimens given in the following pages the Bold-face Roman Italic on page 70 is one of the best for a good, plain, clear, free-hand letter, and is often used with good success on working drawings. Gillott's No. 303 steel pen is the best to use when making this letter free-hand.

The "Yonkers" is a style of letter that is sometimes used for mechanical drawings. It is easy to construct with either F. Soennecken's Round Writing-pens, single point, or the Automatic Shading-pen. But it lacks legibility, and is therefore not a universal favorite.

A good style for "Notes" on a drawing is the "Gothic Condensed" shown on page 70 .

When making notes on a drawing with this letter, the only guides necessary are two parallel lines, drawn lightly in pencil. The letters should be sketched lightly in pencil first, 
and then carefully inked, improving spacing and proportions to satisfy the practiced eye.

\section{FIGURING.}

Great care should be taken in figuring or dimensioning a mechanical drawing, and especially a working drawing.

To have a drawing accurately, legibly, and neatly figured is considered by practical men to be the most important part of a working drawing.

There should be absolutely no doubt whatever about the character of a number representing a dimension on a drawing.

Many mistakes have been made, incurring loss in time, labor, and money through a wrong reading of a dimension.

Drawings should be so fully dimensioned that there will be no need for the pattern-maker or machinist to measure any part of them. Indeed, means are taken to prevent him from doing so, because of the liability of the workman to make mistakes, so drawings are often made to scales which are difficult to measure with a common rule, such as $2^{\prime \prime}$ and $4^{\prime \prime}=$ I $\mathrm{ft}$.

The following books, among the best of their kind, are recommended to all who desire to pursue further the study of "Lettering" : Plain Lettering, by Prof. Henry S. Jacoby, Cornell University, Ithaca, N. Y. ; Lettering, by Charles IV. Reinhardt, Chief Draftsman, Engineering News, New York; Free-hand Lettering, by F. T. Daniels, instructor in C. E. in Tufts College. 
LETTERING AND FIGURING.
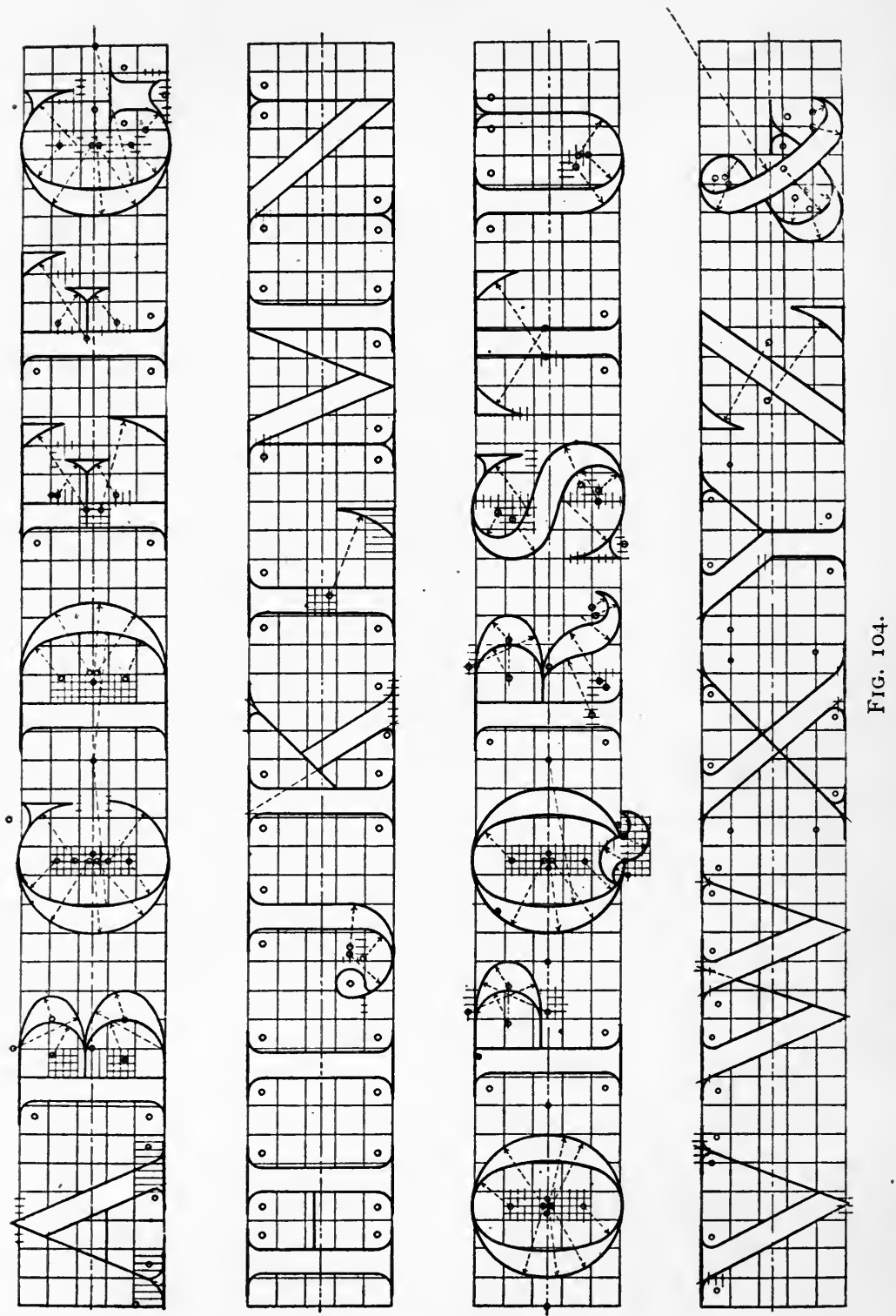

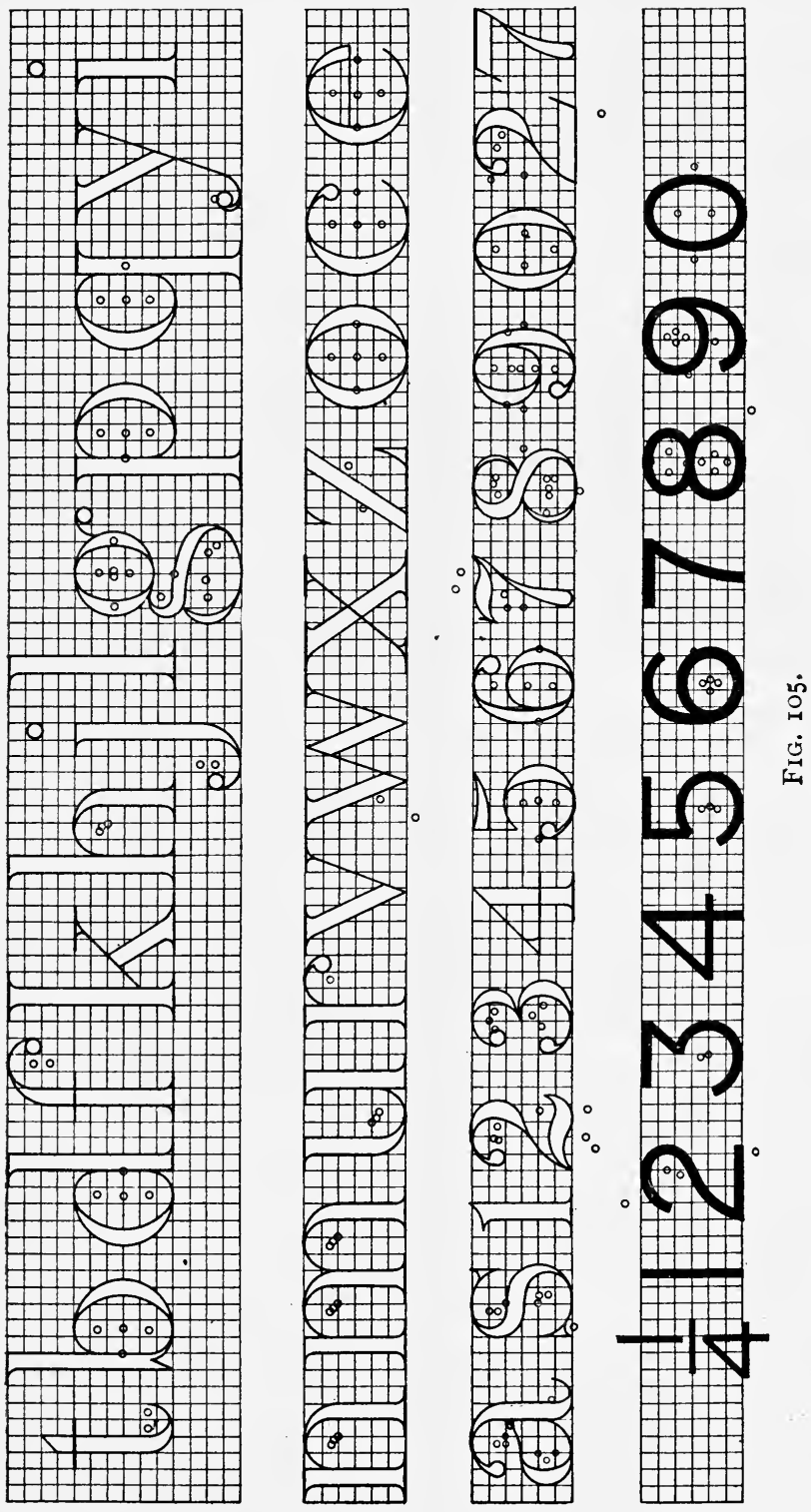

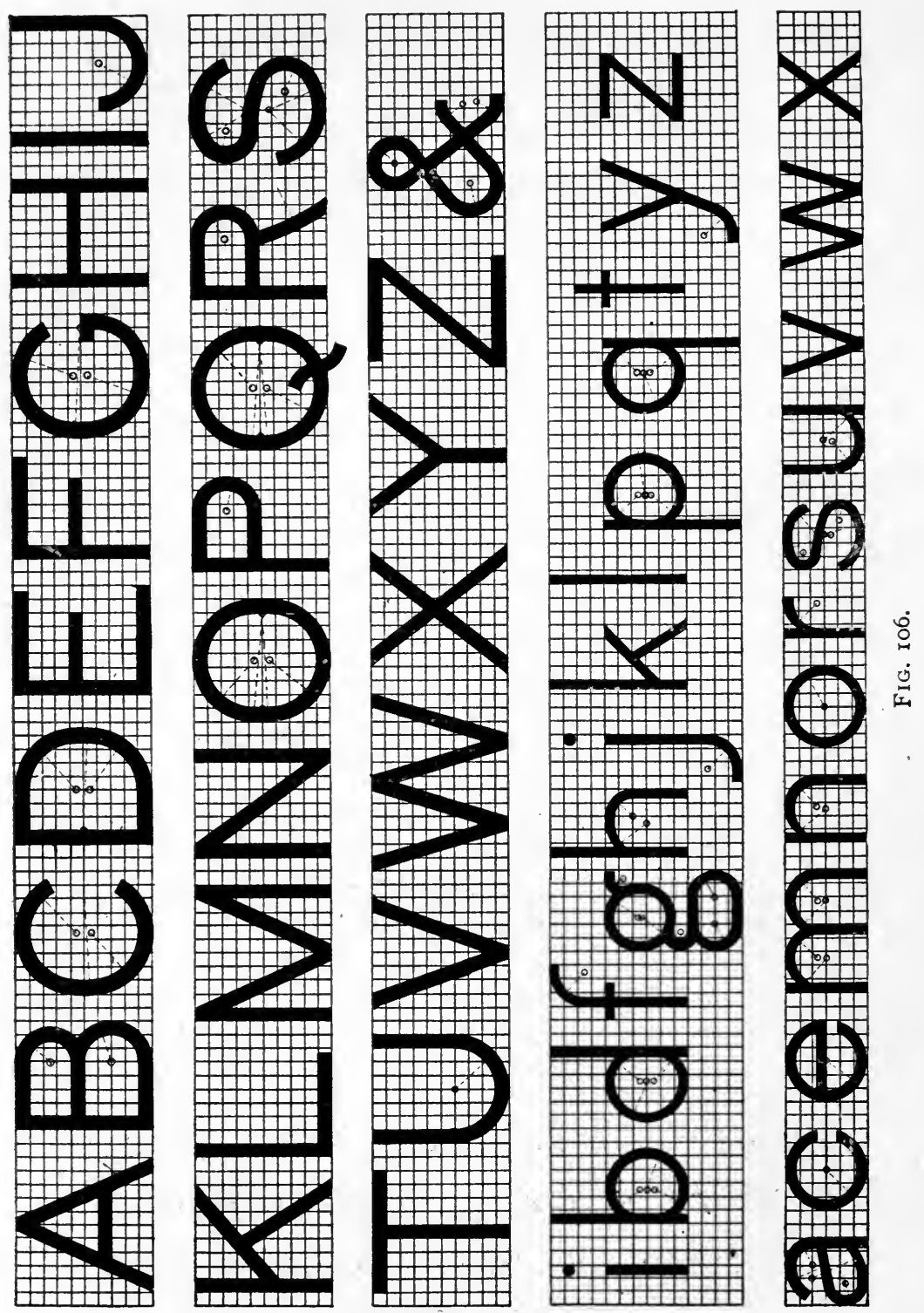


\section{ABCDEFGHIJKLMNOPQRSTUV WX}

- YZ abedefghijklmnopqrsturwxyz

$$
1234567890
$$

\section{ABCDEFGHIJKLMNOPQRSTUV WXYZ abcdefghijlitmnopqrstuww:yz} ABCDEFGHIJKLMNOPQRSTUVWXYZ abcdefghijklm nopqrstuvwxyz $\quad 1234567890$

28-Point Boldface Italic.

\section{ABCDEFGHI.JKLM \\ NOPQRSTUVWXYZ} abcdefighijlilmmopqrstu

\section{vwoxyz $123456 \% 890$}

Two-Line Nonpareil Gothic Condensed. ABCDEFGHIJKLMNOPQRSTUVWXYZ 1234567890

Three-Line Nonpareil Lightface Celtic.

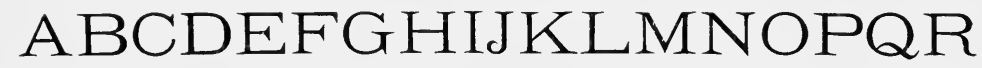
STUVWXYZ abedefghijkl mnopqrstuvwxyz 1234567890 
18-Point Chelsea Circular.

ABCDEFGHIJKLMNOPQRSTUVWX

YZ abcdefghijkImnopqrstuvwxyz 1234567890

18-Point Elandkay.

ABCDEFGHIJKLMNOPQRSTUVWXYZ

1234567890

18-Point Quaint Open.

ABCDEFGIDIRLMNORQRSTUVI 叫XYZ 1234567890

:Arpour Banan

\section{ABCDEFGHIJKLM \\ NOPQRSTUVWXYZ}

abcdefghijklmnopqrstu

vwxyz 1234567890

28-Point Old-Style Italic.

ABCDEFGHIFKLMNOP

QRSTUVWXYZ abcdefg hijklmnopqrstuvwxyz

$$
\text { I } 234567890
$$


12-Point Victoria Italic.

\section{ABCDEFGHIJ KLMNOPQRSTU VWXYZ 1234567890}

I8-Point DeVinne Italic.

\section{ABCDEFGHIJKLMNOPQRSTU $V W X Y Z$ abcdefghijklmnopqrst uVWXYZ 1234567890}

22-Point Gothic Italic.

ABCDEFGHIJKLMNOPQRSTUVWXYZ abcdefghijk/mnopqrstuwwxyz

\section{0}

Double-Pica Program.

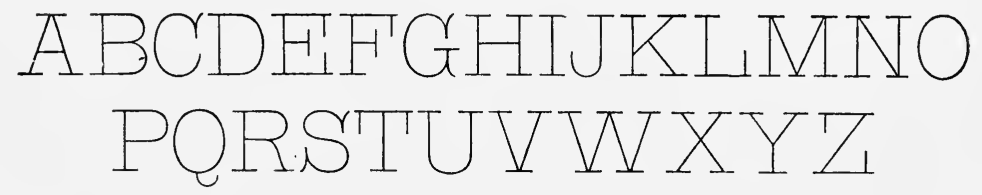

abcdefghijklmnopqrstuv Ixyy 1234567890 
24-Point Gallican.

\section{ABCDEFGHIJKL \\ MNOPQRSTUVW \\ XYZ 1234567890}

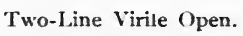

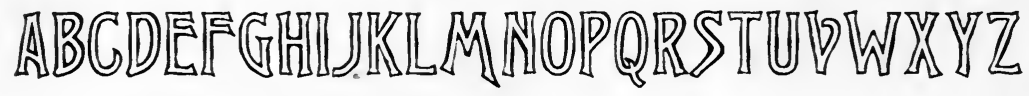

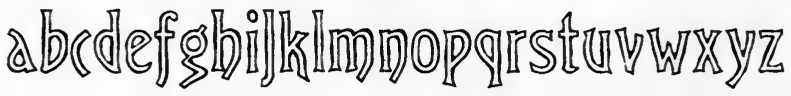
12344567890

22-Point Old-Style Roman.

ABCDEFGHIJKLMNOPQRST

UVWXYZ abcdefghijklmnopqrst uvwxyz I 234567890

\section{ABCDEF(G JJWLI 2YOPQRSTUWW \\ لỹ abedetghijklmnopqr stuvwxyz 1234567890}




\section{CHAPTER V. \\ ORTHOGRAPHIC PROJECTION.}

Orthogkapinc Projection, sometimes called Descriptive Geometry and sometimes simply Projection, is one of the divisions of descriptive geometry; the other divisions are Spherical Projection, Isometric Projection, Shades and Shadows, and Linear Perspective.

In this course we will take up only a sufficient number of the essential principles of Orthographic Projection, Isometric Projection, and Shades and Shade Lines, to enable the student to make a correct mechanical drawing of a machine or other object.

Orthographic Projection is the science and the art of representing objects on different planes at right angles to each other, by projecting lines from the point of sight through the principal points of the object perpendicular to the Plancs of Projection.

There are commonly three planes of projection used, viz., the H.P. or Horizontal Plane, the I. P. or Vertical Plane, and the Pf. P. or Profile Plane.

These planes, as will be seen by Figs. I07 and 109, intersect each other in a line called the $I$. L. or Intersccting Linc, and form four angles, known as the first, second, third, and 
fourth Dihedral Angles. Figs. 107 and I09 are perspective views of these angles.

An object may be situated in any one of the dihedral angles, and its projections drawn on the corresponding coordinate planes.

Problems in Descriptive Geometry are usually worked out in the first angle, and nearly all English draftsmen project their drawings in that angle, but in the United States the third angle is used almost exclusively. There is good reason for doing so, as will be shown hereafter.

We will consider first a few projection problems in the first angle, after which the third angle will be used throughout.

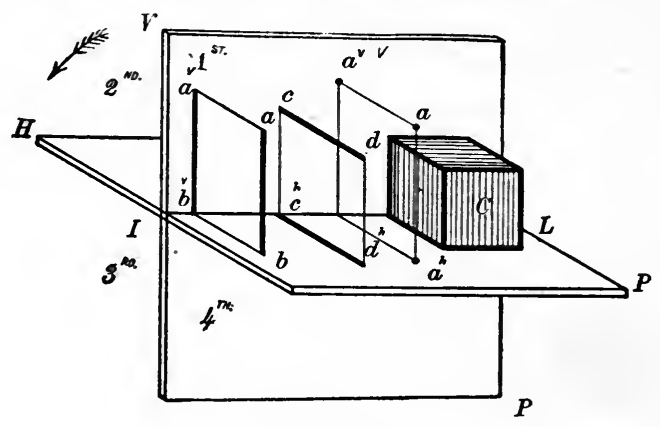

FIG. 107.

H.P., Fig. 107, is the Horizontal Plane, V.P. the Vertical Plane, and I.L. the Intersecting Line.

The Horizontal Projection of a point is where a perpendicular line drawn through the point pierces the H.P.

The Vertical Projection of a point is where a per. line drawn through the point pierces the V.P.

Conceive the point $a$, Fig. I07, to be situated in space $4^{\prime \prime}$ above the H.P. and $3^{\prime \prime}$ in front of the V.P. If a line is passed through the point $a$ per. to H.P. and produced until 
it pierces the H.P. in the point $a^{h}$, $a^{\frac{1}{t}}$ will be the Hor. Proj. of the point $a$.

If another line is projected through the point $a$ per. to the V.P. until it pierces the V.P. in the point $a^{v}, a^{v}$ is the vertical projection of the point $a$.

If now the V.P. is revolved upon its axis I.L. in the direction of the arrow until it coincides with the H.P. and let the H.P. be conceived to coincide with the plane of the drawing-paper, the projections of the point $a$ will appear as shown by Fig. IO8.

The vertical projection $a^{v} 4^{\prime \prime}$ above the I.L. and the horizontal projection $a^{h} 3^{\prime \prime}$ below the I.L. both in the same straight line.

In mechanical drawing the vertical projection $a^{v}$ is called the Eleration and the horizontal projection $a^{h}$ the Plan.

The projections of a line are found in a similar manner, by first finding the projections of the two ends of the line, and joining them with a straight line.

Let $a b$ be a line in space $3 \frac{12^{\prime \prime}}{2}$ long, parallel to the V.P. and perpendicular to the H.P. One end is resting on the H.P. $2 \frac{1}{2}$ " from the V.P.

The points $a$ and $b$ will be vertically projected in the points $a^{v}$ and $b^{v}$. Join $a^{v} b^{v}$. $a^{v} b^{v}$ is the vertical projection of the line $a b$.

When a line is perpendicular to one of the planes of projection, its projection on that plane is a point, and the projection on the other plane is a line equal to the line itself.

$a b$, Fig. 107, is perpendicular to the H.P., therefore its proj. on the H.P. when viewed in the direction $a b$ will be seen to be a point. 
Conceive now the V.P. revelved as before, the V. proj. will be found to be at $a^{v} b^{v}$, Fig. 108, and the H. proj. at the point $a^{h}$.

$c d$, Fig. 107, is a line parallel to the H.P. and perpendicular to the V.P. Its elevation or V. proj. is the point $d^{v}$, Fig. I08, and its plan or $\mathrm{H}$. proj. the line $c^{h} d^{h}$ perpendicular to the Intersecting Line and equal in size to the line itself.

Planes or Plane Surfaces bounded by lines are projected by the same principles used to project lines and points.

Let $a a^{v} b^{v} b$, Fig. 107, be a plane at right angles to and touching both planes of projection.

The elevation of the front upper corner $a$ is projected in the point $a^{v}$. The elevation of the front lower corner $b$ is projected in the point $b^{v}$. Join $a^{v} b^{v} \cdot a^{v} b^{v}$ is the vertical projection of the front edge $a b$ of the plane. The plan of the front

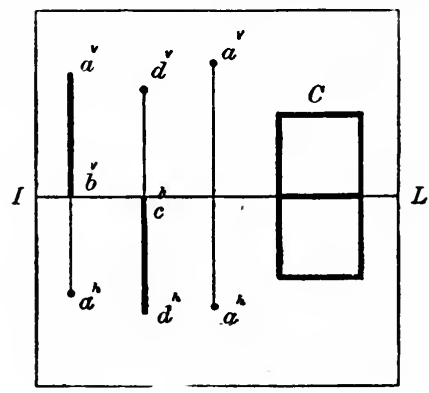

FIG. 108 .

upper corner is projected in the point $b$ and the point $a^{v}$ in the point $b^{v}$. A straight line joining $b b^{v}$ is the plan or horizontal projection of the top edge of the plane.

On the drawing-paper the plan and elevation of the plane $a a^{v} b a$ would be shown as a continuous straight line $a^{v}$ to $a^{b}$ Fig. 108. 
Solids bounded by plane surfaces are projected by means of the same principles used to project planes, lines, and points.

$C$, Fig. IO7, is a cube bounded by six equal sides or surfaces. The top and bottom being parallel to the H.P. and the front and back parallel to the V.P., the vert. proj. is a square above I.L. equal in area to any one of the six faces of the cube. The hor. proj. is a similar square below I.L.

These projections are shown at $C$, Fig. Io8, as they would appear on the drawing-paper.

The foregoing illustrates a few of the simple principles of projection in relation to points, lines, and solids when placed in the first dihedral angle, and we find that the plan is always below and the elevation always above the I.L.

Let us now consider the same problems when situated in the third angle. The point $a$, Fig. Io9, is behind of the V.P.

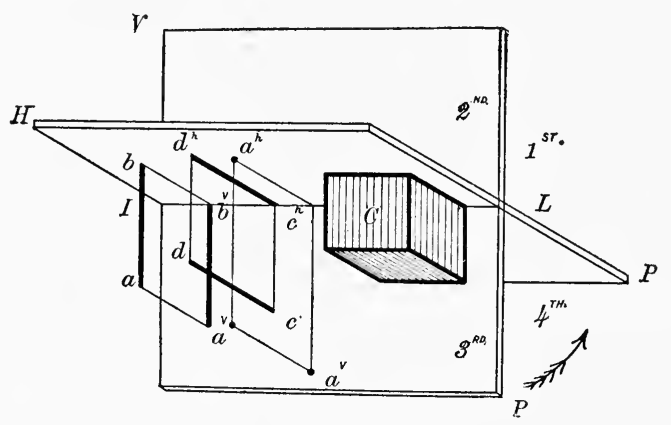

FIG. IOg.

and below the H.P. Draw through a perpendiculars to the plane of projection. The Hor. proj. is found at $a^{h}$ and the vert. proj. at $a^{v}$.

Conceive again the V.P. to be revolved in the direction of the arrow until it coincides with the H. P. The hor. proj. 
will then appear at $a^{h}$ above the I.L. and the vert. proj. at $a^{v}$ below the I.L., Fig. Iro. And so with the lines, the planes, and the solids.

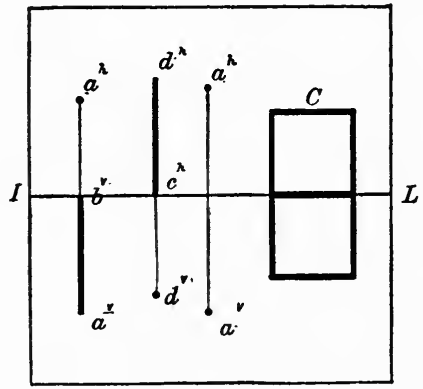

FIG. IIO.

In order to still further explain the use of the planes of projection, with regard to objects placed in the third angle, let us suppose a truncated pyramid surrounded by imaginary planes at right angles to each other, as shown by Fig. III.

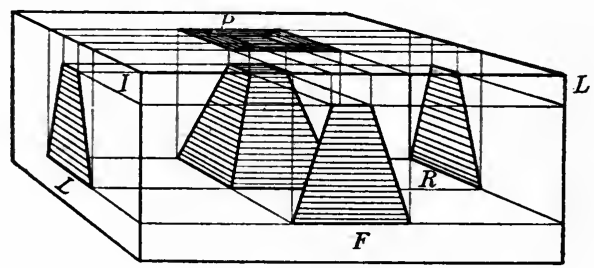

FIG. III.

With a little attention it will easily be discerned that the pyramid is situated in the third dihedral angle, and that in addition to the $\mathrm{V}$. and $\mathrm{H}$. planes, we have passed two profile planes at right angles to the $\mathrm{V}$. and $\mathrm{H}$. planes, one at the righthand and one at the left.

When the pyramid is viewed orthographically through each of the surrounding planes, four separate views are had, 
exactly as shown by the projections on the opposite planes, viz., a Front View, Elevation, or Vert. Proj. at F.; a Righthand View, Right-end Elevation, or Right-profile Projection at R.; a Left-hand View, Left-end Elevation, or Left-profile Projection at L.; a Top View, Plan or H. Proj. at P.

If we now consider the V.P. and the right and left profile planes to be revolved toward the beholder until they coincide, using the front intersecting lines as axes, the projections of the pyramid will be seen as shown by Fiog. I I2, which when the

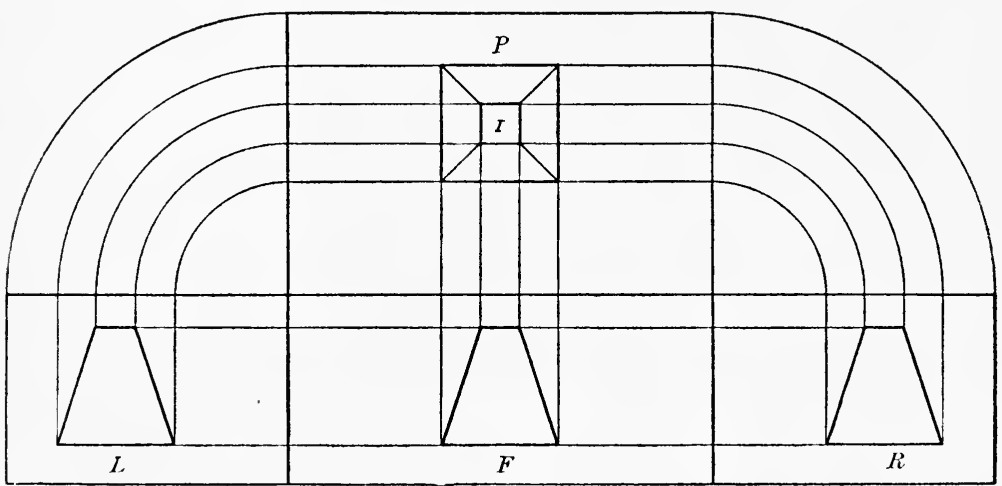

FIG. II2.

imaginary planes and projecting lines have been removed, will bc a True Drawing or Orthographic Projection of the truncated pyramid.

\section{NOTATION.}

In the drawings illustrating the following problems and their solutions the given and required lines are shown wide and black. Hiddcn lines are shown broken into short dashes a little narrower than the zisible lines. Construction or projection lines are drawn with very narrow full or continuous black lines. 
When convenient very narrow, continuous blue lines are sometimes used.

The Horizontal Plane is known as the H.P., the Vertical Plane as V.P. and the Profile Plane as Pf.P.

A point in space is designated by a small letter or figure, their projection by the same letters or figures with small $h$ or $v$ written above for the horizontal or vertical projection respectively.

In some complicated problems where points are designated by figures their projections are named by the same figures accented.

Drawings should be carefully made to the dimensions given, the scale to be determined by the instructor.

The student should continually endeavor to improve in inking straight lines, curves, and joints.

In solving the following problems the student should have a model of the co-ordinate planes for his own use. This can be made by taking two pieces of stiff cardboard and cutting a slot in the center of one of them large enongh to pass the folded half of the other through it; when unfolding this half a model will be had like that shown by Fig. I07 or I09.

All projections shall now be made from the third, dihedral angle.

Рвов. I.-A point $a$ is situated in the third dihedral angle, $\mathrm{I}^{\prime \prime}$ below the H.P. and $3^{\prime \prime}$ behind the V.P.

It is required to draw its vertical and horizontal projections.

Draw a straight line $a^{h} a^{v}$, Fig. I13, perpendicular to I.L. and measure off the point $a^{v} \mathrm{I}^{\prime \prime}$ below I.L. and the point $a^{h}$ $3^{\prime \prime}$ above I.L. 
$a^{v}$ is the vertical and $a^{h}$ the horizontal projection in the same straight line $a^{v} a^{h}$.

The student should demonstrate this with his model.

PROB. 2.-Draw two projections of a line $3^{\prime \prime}$ long parallel to both planes, $\frac{3}{4}$ " below the H.P. and $2^{\prime \prime}$ behind the V.P.

As the line is parallel to both planes, both projections will be parallel to the I.L.

Draw $a^{v} b^{v}$ the vert. proj. of the line $3^{\prime \prime}$ long, Fig. I I 4 , parallel to I.L. and $\frac{3^{\prime \prime}}{4}$ below it. Draw the hor. proj. $2^{\prime \prime}$ above the I.L. and parallel to it, making it the same length as the

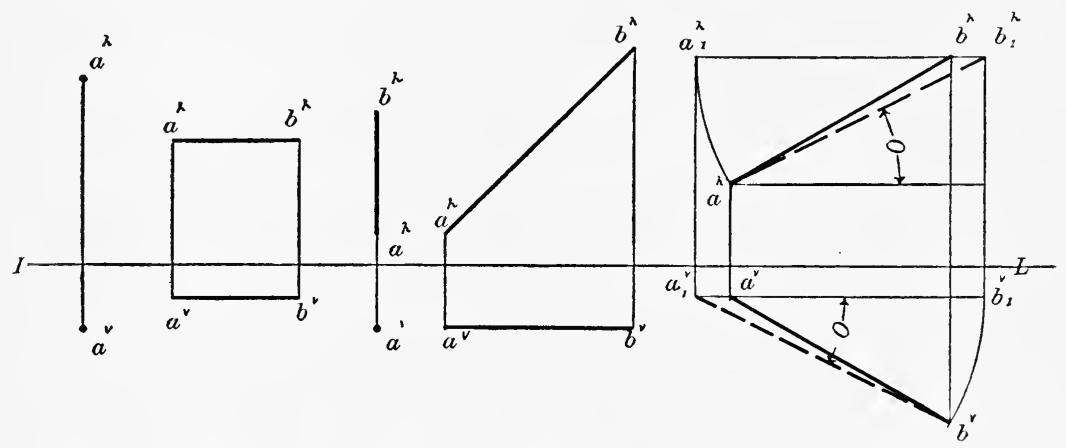

FIG. II3. FIg. II4. FIG. II5. FIg. II6.

FIG. II7.

vert. proj. by drawing lines perpendicular to I.L. from the points $a^{v}$ and $b^{v}$ to $a^{h}$ and $b^{h}$.

PROB. 3.- To draw the hor. and vert. projs. of a straight line $3^{\prime \prime}$ long, per. to the vert. plane, Fig. I 15.

As the line is per. to the vert. plane the vert. proj. will be a point below the I.L. and the hor. proj. will be parallel to the horizontal plane and per. to I.L.

PROB. 4.- To draw the plan and elevation of a straight line $6^{\prime \prime}$ long making an angle of $45^{\circ}$ with the vert. plane and and par. to the hor. plane, Fig. I 6. 
The plan or hor. proj. will be above the I.L. and make an angle of $45^{\circ}$ with it. The elevation or vert. proj. will be below and par. to I.L.

Draw from the point $a^{h}$ at any convenient distance from I.L. a straight line $a^{h} b^{h} 6^{\prime \prime}$ long, making an angle $45^{\circ}$ with I.L.

Draw $a^{v} b^{v}$ par. to I.L. at a convenient distance below it. The length of the elevation or vert. proj. is determined by dropping perpendiculars from the end of the hor. proj. $a^{h} b^{h}$ to the points $a^{v} b^{v}$.

Prob. 5, Fig. I I7.-To find the true length of a straight line oblique to both planes of projection and the angle it makes with these planes.

$a^{v} b^{v}$ and $a^{h} b^{h}$ are the projections of a straight line oblique to V.P. and H.P. Using $a^{v}$ as a pivot, revolve the line $a^{v} b^{v}$ until it becomes parallel to I.L. as shown by $a^{v} b_{1}{ }^{v}$. From the point $b_{1}^{v}$ erect a per. Through the point $b^{h}$ draw a line par. to I.L. cutting the per. in the point $b_{1}{ }^{h}$.

The broken line $a^{k} b_{1}{ }^{h}$ is the true length of the line $a b$, and the angle $o$ is the true angle which the line makes with V.P.

To find the angle it makes with H.P.:

Using $b^{h}$ as a pivot, revolve the line $b^{h} a^{h}$ until it becomes par. to I.L. as shown by $b^{h} a_{1}{ }^{h}$. From the point $a_{1}{ }^{h}$ drop a per. Through the point $a^{y}$ draw a line par. to I.L. intersecting the per. at the point $a_{1}{ }^{0} o$ is the angle which the line $a b$ makes with H.P. and the broken line $a_{1}^{v} b^{v}$ is again its true length.

PROB. 6, Fig. II8.-To project a plane surface of given size, situated in the third angle and par. to the V.P.

Let $a b c d$ be the plane surface $3^{\prime \prime}$ long $\times 2^{\prime \prime}$ wide. If we conceive lines to be projected from the four corners of the 
plane surface to the V.P. and join them with straight lines we will have its V. projection $a^{v} b^{v} c^{v} d^{v}$ and shown by Fig. I I 8 . And as the plane surface is par. to the V.P. it must be per to the H.P. since the planes of projection are at right angles to each other. So the plan or $H$. projection will be a straight line equal in length to one of the sides of the plane surface.

At a convenient distance above I.L. draw a straight line, and from the points $a^{v} b^{v}$ project lines at right angles to I.L., cutting the straight line in the points $a^{h} b^{h}$ The line $a^{h} b^{h}$ is the hor. proj. of the plane surface $a b c d$. .

PROB. 7, FiG. II 8.- To draw the projections of a plane surface of given dimensions when situated in the third angle perpendicular to the H.P. and making an angle with the V.P.

Let the plane surface be $3^{\prime \prime} \times 2^{\prime \prime}$ as before and let the angle it makes with V.P. be $60^{\circ}$.

To draw the plan:

At a convenient distance above I.L. and making an angle of $60^{\circ}$ with it, draw $a^{h} b_{1}{ }^{h}$, Fig. I I $8,2^{\prime \prime}$ long. From $b_{1}{ }^{h}$ drop a per. cutting $a^{v} b^{v}$ in the point $b_{1}{ }^{v}$ and $c^{v} d^{v}$ in the point $d_{1}{ }^{\prime}$, then the rectangle $a^{v} b_{1}^{v} d_{1}^{v} c^{v}$ will be the vert. proj. or elevation of the plane surface $a b c d$.

Prob. 8, Fig. I I9.-To draw the projections of the same plane surface (I) when parallel to the H.P., (2) when making an angle of $30^{\circ}$ with H.P. and per. to V.P., (3) when making an angle of $60^{\circ}$ with H.P. and per. to V.P., and (4) when per. to both planes.

Fig. I I9 shows the projections; further explanations are unnecessary.

Prob. 9, Figs. I I9 AND I 20.-To draw the projections of 
the same plane surface when making compound angles with the planes of projection.

Let the plane make an angle of $30^{\circ}$ with H.P., as in the second position of Prob. 8, Fig. I I9, and in addition to that, revolve it through at angle of $30^{\circ}$. First, draw the plane parallel to H.P., as shown by $a^{h} c^{h} b^{h} d^{h}$, Fig. I I9, the true size of the plane.

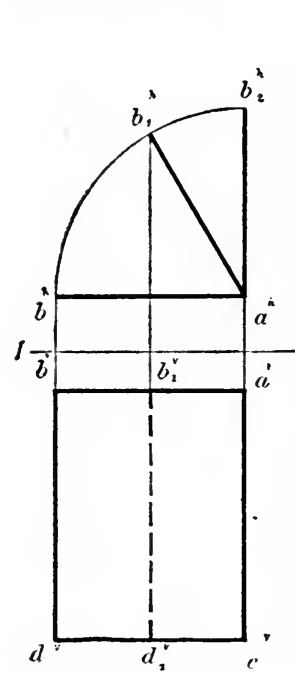

FIG. II 8 .

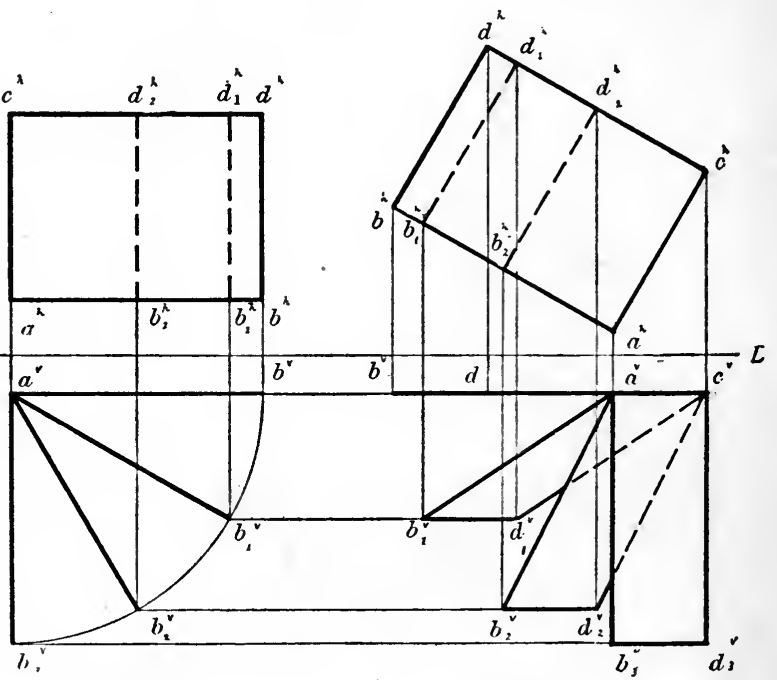

FIG. II9.

FIG. 120.

Its elevation will be the straight line $a^{v} b^{v}$ parallel to I.L. Next revolve $a^{v} b^{v}$, using $a^{v}$ as a pivot, through an angle of $30^{\circ}$, to the position $a^{v} b_{1}^{v}$, which is its vert. proj. when making an angle of $30^{\circ}$ with H.P. Its plan is projected in $a^{h} b_{1}{ }^{h} c^{h} d_{1}^{h}$.

Now as the plane is still to make an angle of $30^{\circ}$ with H.P. after it has been revolved through an angle of $30^{\circ}$. with relation to the V.P., its hor. proj. will remain unchanged.

IVith a piece of celluloid or tracing-paper trace the hor. proj. $a^{h} b_{1}{ }_{1} c^{h} d_{1}{ }^{h}$, lettering the points as shown, and revolve the 
tracing through the angle of $30^{\circ}$, or, which is the same thing, place the tracing so that the line $a^{h} c^{h}$ will make an angle of $60^{\circ}$ with I.L., and with a sharp conical-pointed pencil transfer the four points to the drawing-paper and join them by straight lines, as shown by Fig. I20.

And as the line $a^{h} c^{h}$ retains its position relative to H.P. after the revolution, its elevation will be found at $a^{v} c^{v}$, Fig. I20, in a straight line drawn through $a^{7} b^{*}$, Fig. I I 9 , intersecting perpendiculars from $a^{h} c^{h}$, Fig. I20. And the vert. proj. of the points $b_{1}{ }^{h} d_{1}^{h}$ will be found at $b_{1}{ }^{2} d_{1}{ }^{v}$, Fig. 120, in a straight line drawn through $b_{1}{ }^{\nu}$, Fig. I I 9, parallel to I.L. and intersecting pers. from $b_{1}^{h} d_{1}^{h}$, join with straight lines the points $a^{v} b_{1}^{v} c^{v} d_{1}^{v}$.

Draw the projections of the plane when making an angle of $60^{\circ}$ with H.P. and revolved through an angle of $30^{\circ}$ with relation to $\mathrm{V} . \mathrm{P}$.

Draw the projections of the plane when making an angle of $60^{\circ}$ with the V.P. and per. to the H.P., Fig. I2O.

PROB. 10. - To draw the projections of a plane surface of hexagonal form in the following positions: (I) When one of its diagonals is par. to the V.P. and making an angle of $45^{\circ}$ with the H.P. (2) When still making an angle of $45^{\circ}$ with the H.P. the same diagonal has been revolved through. an angle of $60^{\circ}$.

Draw the hexagon $1^{h} 2^{h} 3^{h} 4^{h} 5^{h} 6^{h}$, Fig. I2I, at any convenient distance above I.L., making the inscribed circle $=2 \frac{1}{2}{ }^{\prime \prime}$. This will be its hor. proj. and $2^{v} 4^{v} 6^{v} 1^{v}$ its vert. proj., the diagonal $\mathbf{I}^{h} 2^{h}$ being par. to both planes of proj. With $1^{v}$ as an axis revolve $6^{v} 4^{v} 2^{v}$ through an angle of $45^{\circ}$. Through the points $2{ }_{1}{ }^{v} 4_{1}{ }^{v} \sigma_{1}{ }^{v}$ erect pers. to the points $6_{1}{ }^{h} 5_{1}{ }^{h} 4_{1}{ }^{h} 3_{1}{ }^{h}$ and $2_{1}{ }^{h}$ 
and join them with straight lines. These are the projs. in the first position. Now trace the hor. proj, $1^{h}, 2_{1}{ }^{h}$, etc., on a piece of celluloid or tracing-paper and revolve the tracing until the diagonal $1^{h} 2_{1}{ }^{h}$ makes an angle of $60^{\circ}$ with the I.L., Fig. 122. Next draw pers. from the 6 points of the hexagonal plane to intersect hors. from the corresponding points of the elevation in Fig. $12 \mathrm{I}$, join the points of intersection with

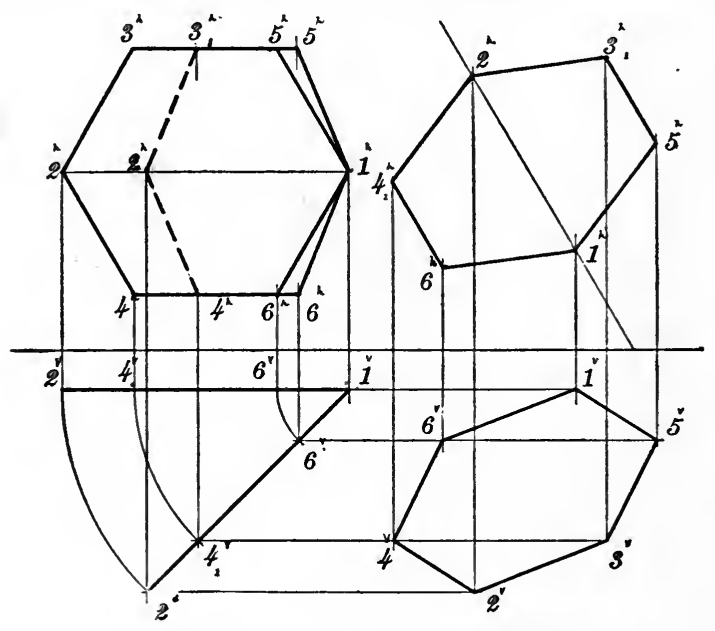

FIG. 12I.

FIG. 122.

straight lines, and so complete the projections of the second position, Fig. I22.

Prob. I I, Figs. I23 AND 124.-Draw the projs. of a circular plane (I) when its surface is par. to the vert. plane, (2) when it makes an angle of $45^{\circ}$ with the V.P., and (3) when still making an angle of $45^{\circ}$ with the V.P. it has been revolved through an angle of $60^{\circ}$.

First position: Draw the circular plane $1^{v}, 2^{v}, 3^{v}, 4^{v}$, etc., Fig. 123 , below the I.L. with a radius $=1 \frac{1}{2}$ " and divide and figure it as shown. 
Since the plane is par. to V.P. its hor. proj. will be a straight line $\mathrm{I}^{h}, 2^{h}, \ldots$. etc.

For the second position revolve the said hor. proj. through the required angle of $45^{\circ}$ to the position $a^{h} \ldots \mathrm{I}_{1}{ }^{h}$, Fig. I 23 , and through each division in $\mathrm{I}^{h} \ldots a^{h}$ draw arcs cutting $a^{h} \ldots \mathrm{I}^{h}$ in points $2^{h} 3^{h} \ldots$ This is the hor. proj. of the plane when making an angle of $45^{\circ}$ with the V.P.

The elevation is found by dropping pers. from the points in the hor. proj. $a^{h} \ldots \mathrm{I}_{1}{ }^{h}$ to intersect hor. lines drawn through the correspondingly numbered points in the eleva-

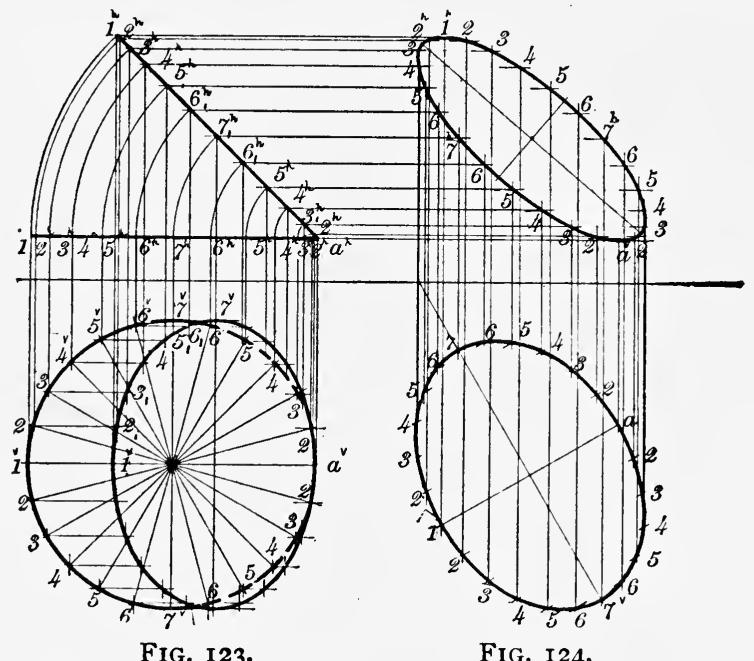

tion and through these intersections draw the elevation or vert. proj. of the second position.

For the third position make a tracing of the elevation of the second position, numbering all the points as before, and place the tracing so that the diameter $7^{v} 7^{v}$ makes the required angle of $60^{\circ}$ with the I.L. and transfer to the drawing-paper. 
The result will be the elevation of the third position shown below the I.L., Fig. 124. Its hor. proj. is found by drawing pers. through the points $\mathrm{I}, 2,3,4 \ldots$ to intersect hors. drawn through the corresponding points in the hor. proj. of the $2 \mathrm{~d}$ position and through these intersections draw the plan or hor. proj. of the third position, Fig. 124.

Prob. I2, Fig. 125.-Draw the projs. of a regular hexagonal prism, $3^{\prime \prime}$ high and having an inscribed circle of $4 \frac{5}{8}^{\prime \prime}$ diam.: (I) When its axis is par. to the V.P. (2) Draw the true form of a section of the prism when cut by a plane passing through it at an angle of $30^{\circ}$ with its base.

Draw the projection of a section when cut by a plane passing through $X X$, Fig. 125, per. to both planes of proj.

The drawing of the I.L. may now be omitted.

For the plan of the first part of this prob. draw a circle with a radius $=$ to $2 \frac{5}{16}$ " , and circumscribe a hexagon about it, as shown by $a^{h}, b^{h}, b^{h}$, etc., Fig. I25. To project the elevation, draw at a convenient distance from the plan a hor. line par. to $a^{h} d^{i n}$, and $3^{\prime \prime}$ below it another line par. to it. From the points $a^{h} b^{h} c^{h} d^{h}$, drop pers. cutting these par. lines in the points $a^{v} b^{v} c^{v} d^{v}$, thus completing the elevation of the prism.

Second condition: Draw the edge view or trace of the cutting plane $\mathrm{I}^{\prime} 4^{\prime}$, making an angle of $30^{\circ}$ with the base of the prism, locating the lower end $4^{\prime}$ one-half inch above the base; parallel to ${ }^{\prime}{ }^{\prime}{ }^{\prime}$, and at a convenient distance from it draw a straight line $\mathrm{I}, 4$; at a distance of $2 \frac{5}{16}{ }^{\prime \prime}$ on each side of $\mathrm{I}, 4$ draw lines 3, 2 and 5, 6 parallel to I, 4, and through the points $I^{\prime} 2^{\prime} 3^{\prime} 4^{\prime}$ let fall pers. cutting these three par. lines in the points I, 2, 3, 4, 5, 6; join these points by straight lines 
as shown, and a true drawing of the section of the prism as required will result.

For the third condition of the problem :

Let $X X$ be the edge view of the cutting plane and conceive that part of the prism to the right of $X X$ to be removed.

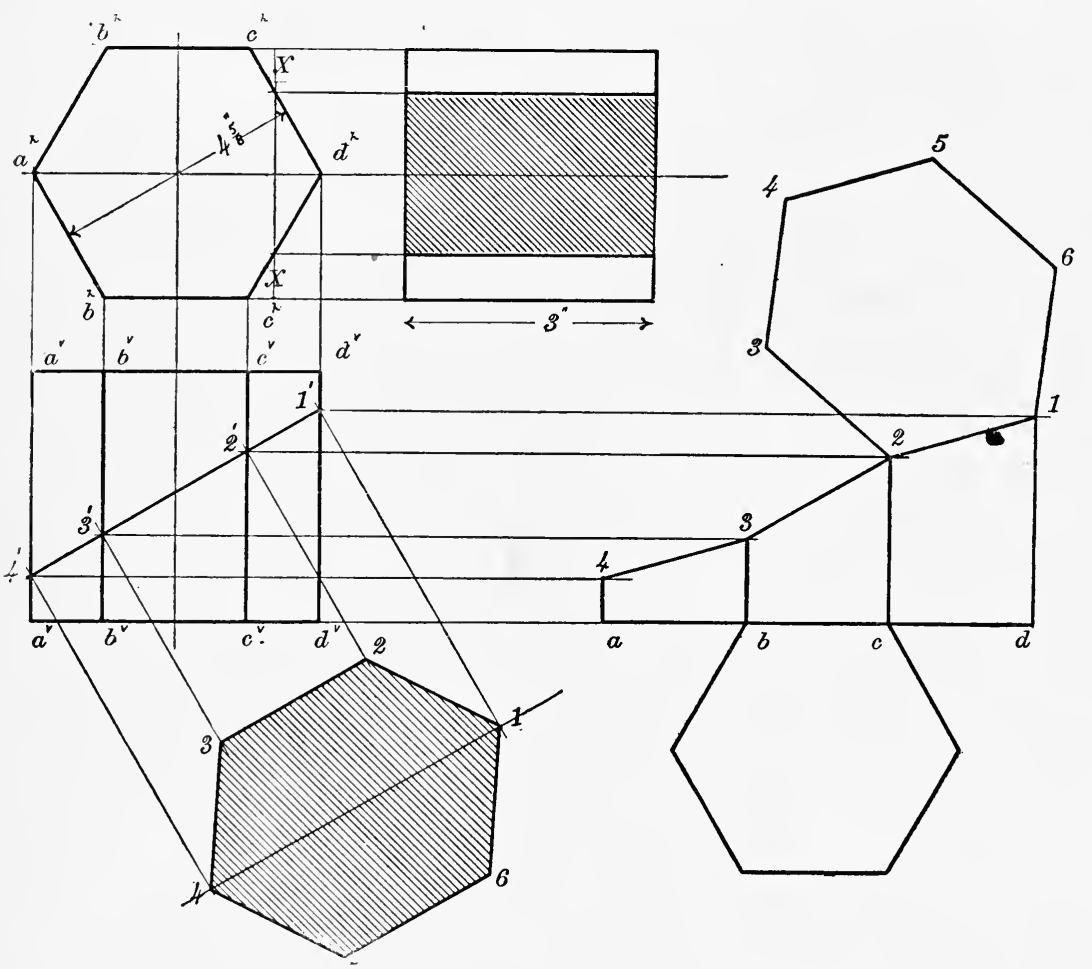

FIG. โ25.

FIG. I 26.

From the hor. proj. of the prism draw a right-hand elevation or profile proj., and through the points $X X$ draw the lines enclosing the section, and hatch-line it as shown.

PRoв. 13.-To draw the development of the lower part of the prism in the elevation of the last problem. 
To the right of the elevation in Fig. 125, prolong the base line indefinitely and lay off upon it the distances $a b, b c, c d$, etc., Fig. 126, each equal in length to a side of the hex. At these points erect pers., and through the points ${ }^{\prime} 2^{\prime} 3^{\prime} 4^{\prime}$ draw hor. lines intersecting the pers. in $4,3,2$, I, etc. At $b c$ draw the hex. $a^{h} b^{h} b^{h}, c^{h} c^{h}, d^{h}$ of the last prob. for the base, and at I, 2 draw the section I, 2, 3, 4, 5, 6 for the top.

Prob. I4, FIG. 127.- To draw the projs. of a right cylinder $3^{\prime \prime}$ diam. and $3^{\prime \prime}$ long. (1) When its axis is per. to the H.P. (2) Draw the true form of a section of the cylinder, when cut by a plane per. to the V.P. making an angle of $30^{\circ}$ with the H.P. (3) Draw a development of the upper part of the cyl.

For the plan of the first condition, describe the circle $\mathrm{I}^{\prime}$, $2^{\prime}$, etc., with a radius $=1 \frac{1}{2}{ }^{\prime \prime}$ and from it project the elevation, which will be a square of $3^{\prime \prime}$ sides.

For the second condition: Let 1,7 be the trace of the cutting plane, making the point $7, \frac{1}{2}{ }^{\prime \prime}$ from the top of the cyl. Divide the circle into 12 equal parts and let fall pers. through these divisions to the line of section, cutting it in the points $\mathrm{I}, 2,3,4$, etc. Parallel to the line of section $\mathrm{I}, 7$ draw $\mathrm{I}^{\prime \prime} 7^{\prime \prime}$ at a convenient distance from it, and through the points I, 2, 3, 4, etc., draw pers. to I, 7 , intersecting and extending beyond $\mathrm{I}^{\prime \prime} 7$. Lay off on these pers. the distances $6{ }^{\prime \prime}{ }^{\prime \prime}=$ $6^{\prime} 8^{\prime}$, and $5^{\prime \prime} 9^{\prime \prime}=5^{\prime} 9^{\prime}$, etc., and through the points $2^{\prime \prime}, 3^{\prime \prime}$, $4 "$. etc., describe the ellipse.

For the development: In line with the top of the elevation draw the line $g^{\prime} g^{\prime \prime}$ equal in length to the circumference of the circle, and divide it into 12 equal parts $a^{\prime}, b^{\prime}$, etc., $a^{\prime}, b^{\prime \prime}$, etc. Through these points drop pers. and through the points 
1,2 , 3, etc., draw hors. intersecting the pers. in the points I, 2, 3, etc., and through these points draw a curve.

Tangent to any point on the straight line draw a $3^{\prime \prime}$ circle for the top of the cyl. and tangent to any suitable point on the curve transfer a tracing of the ellipse.

PROB. I 5, FIG. I 28. - Draw the projections of a right cone $7^{\prime \prime}$ high, with a base $6^{\prime \prime}$ in diam., pierced by a right cyl. $2^{\prime \prime}$ in

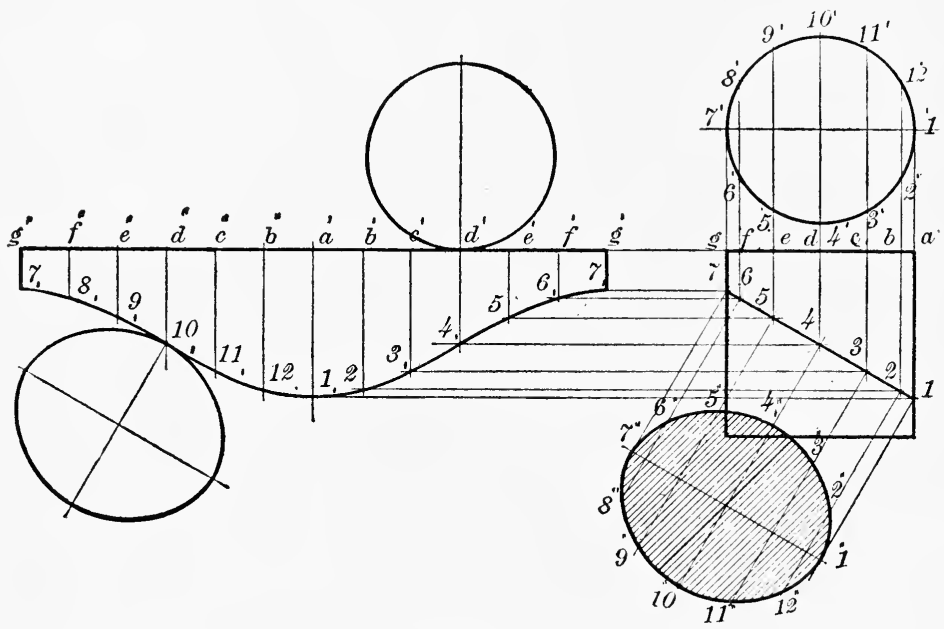

FIG. 127.

diam. and $5^{\prime \prime}$ long their axes intersecting at right angles $3^{\prime \prime}$ above the base of the cone and par. to V.P. Draw first the plan of the cone with a radius $=3^{\prime \prime}$.

At a convenient distance below the plan draw the elevation to the dimensions required.

$3^{\prime \prime}$ above the base of the cone draw the center line of the cyl. $C D$, and about it construct the elevation of the cyl., which will appear as a rectangle $2^{\prime \prime}$ wide and $2 \frac{1}{2}$ " each side of the axis of the cone. The half only appears in the figure. 
To project the curves of intersection between the cyl. and cone in the plan and elevation: Draw to the right of the cyl. on the same center line a semicircle with a radius equal that of the cyl. Divide the semicircle into any number of parts,

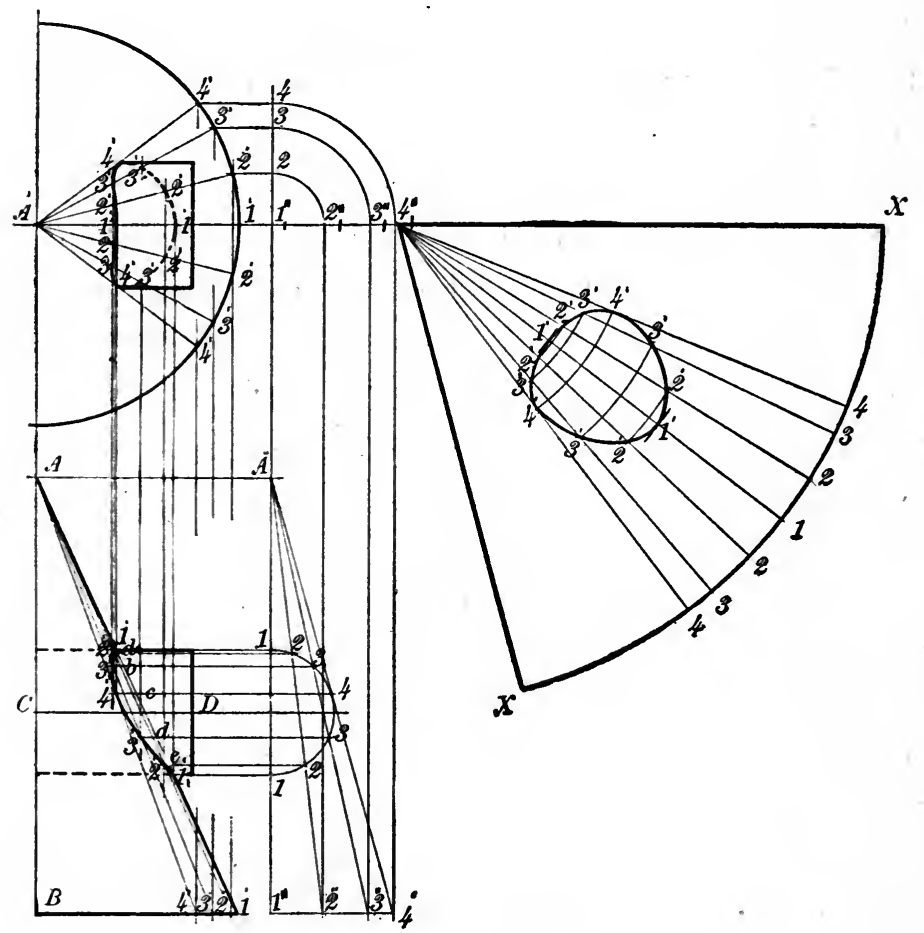

- Fig. 128.

FIG. 129.

as I, 2, 3, 4, etc. Through I, I draw the per. $A^{\prime \prime} \mathrm{I}^{\prime \prime}$ equal in length to the height of the cone, and through $A^{\prime \prime}$ draw the line $A^{\prime \prime} 4^{\prime \prime}$ tangent to the semicircle at the point 4 , and through - the other divisions of the semicircle draw lines from $A^{\prime \prime}$ to the line $1^{\prime \prime} 4^{\prime \prime}$, meeting it in the points $3^{\prime \prime} 2^{\prime \prime}$.

From all points on the line $I^{\prime \prime} 4^{\prime \prime}$, viz., I" ${ }^{\prime \prime} 2^{\prime \prime} 3^{\prime \prime} 4$, erect 
pers. to the center line of the plan, cutting it in the points $\mathrm{I}_{1}{ }^{\prime \prime} 2_{1}{ }^{\prime \prime} 3_{1}{ }^{\prime \prime} 4_{1}{ }^{\prime \prime}$, and with $\mathrm{I}_{1}{ }^{\prime \prime}$ as the center draw the arcs $2_{1}{ }^{\prime \prime}-2$, $3_{1}{ }^{\prime \prime}-3,4{ }_{1}{ }^{\prime \prime}-4$ above the center line of the plan, and through the points $2,3,4$ draw hors. to intersect the circle of the plan in the points $2^{\prime} 3^{\prime} 4^{\prime}$, and lay off the same distances on the other side of the center line of the plan in same order, viz., $2^{\prime} 3^{\prime} 4^{\prime}$. Through each of these points on the circumference of the circle of the plan draw radii to its center $A^{\prime}$, and through the same points also in the plan let fall pers. to the base of the elevation of the cone, cutting it in the points $2^{\prime} 3^{\prime} 4^{\prime}$; and from the apex $A$ of the elevation of the cone draw lines to the points $2^{\prime} 3^{\prime} 4^{\prime}$ on the base. Hor. lines drawn through the points of division 2, 3,4 on the semicircle will intersect the elements $A-2^{\prime}, A-3^{\prime}$, $A-4^{\prime}$ of the cone in the points $2^{\prime} 3^{\prime} 4^{\prime}$; these will be points in the elevation of the curve of intersection between the cylinder and the cone.

The plan of the curve is found by erecting pers. through the points in the elevation of the curve to intersect the radial lines of the plan in correspondingly figured points, through which trace the curve as shown. Repeat for the other half of the curve.

Prob. 16, Fig. I29.-To draw the development of the half cone, showing the hole penetrated by the cyl.

With center $4_{1}^{\prime \prime}$, Fig. I29, and element $A_{\mathrm{I}}{ }^{\prime}$ of the cone, Fig. 128, as radius, describe an arc equal in length to the semicircle of the base of the cone. Bisect it in the line $4_{1}^{\prime \prime} I$, and on each side of the point I lay off the distances 2, 3, 4, equal to the divisions of the arc in the plan Fig. 128 , and from these points draw lines to $4^{\prime \prime}$, the center of the arc. Then with radii $A-a, b, c, d, e$, respectively, on the elevation Fig. I28, 
and center $4{ }_{1}^{\prime \prime}$ draw arcs intersecting the lines drawn from the arc $X X$ to its center $4_{1}^{\prime \prime}$. Through the points of intersection draw the curve as shown by Fig. 129.

Prob. I7, Fig. I30.-To draw the development of the half of a truncated cone, given the plan and elevation of the cone.

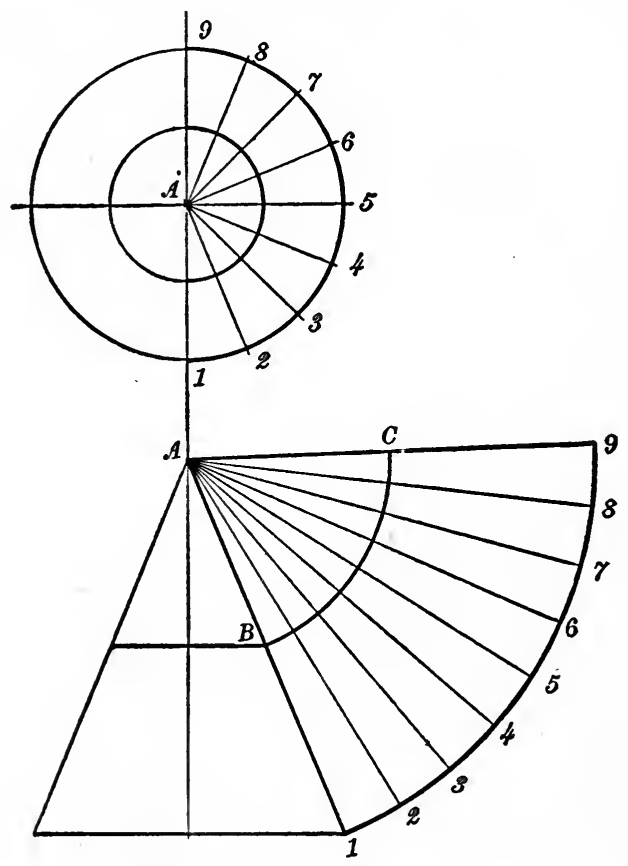

FIG. I30.

Divide the semicircle of the plan into any number of parts, then with $A$ as center and $A \mathrm{I}$ as radius, draw an arc and lay off upon it from the point I the divisions of the semicircle from I to 9 , draw $9 A$. Then with center $A$ and radius $A B$ draw the arc $B C . \quad$ I $B C_{9}$ is the development of the half of the cone approximately. 
PROB. IS, FIG. I31.- To draw the curve of intersection of a small cyl. with a larger. To the left of the center-line of Fig. I 3 I is a half cross-section, and to the right a half elevation. of the two cyls.

Draw the half plan of the small cyl., which will be a semicircle, and divide it into any convenient number of parts, say 12.

From each of these divisions drop pers.

On the half cross-section these pers. intersect the circumference of the large cyl. in the points $I^{\prime}, 2^{\prime}$, etc. Through

FIG. I34.

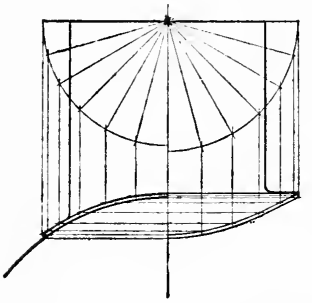

FIG. I33.

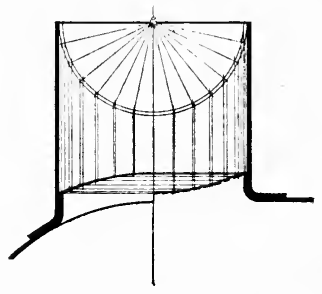

$\begin{array}{lllllllllllll}6 & 5 & 4 & 3 & 2 & 1 & 4 & 1 & 2 & 3 & 4 & 5 & 6\end{array}$

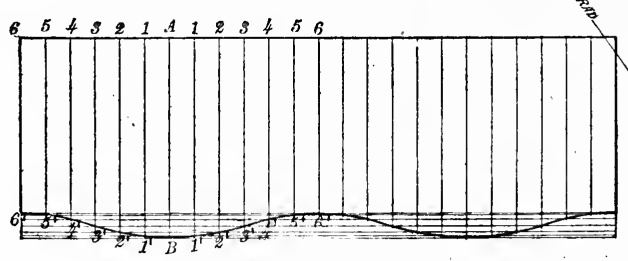

FI(i. I32.
FIG. Ij工.

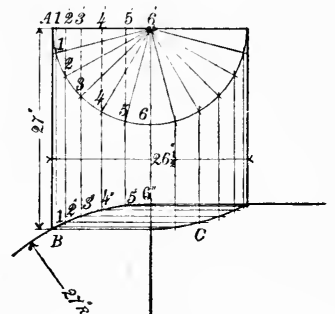

these points draw hors. to intersect in corresponding points the pers. on the half elevation. Through the latter points draw the curve of intersection $C$.

PROB. I9.-To draw the development of the smaller cyl. of the last prob.

Draw a rectangle, Fig. I 32 , with sides equal to the circum- 
ference and length of the cyl. respectively, and divide it into 24 equal parts.

Make $A B, \mathbf{I}^{\prime} \mathrm{I}^{\prime}, 33^{\prime}$, etc., Fig. $\mathbf{1} 32$, equal to $A B, \mathbf{I}^{\prime} \mathbf{I}^{\prime \prime}$, $2^{\prime} 2^{\prime \prime}, 3^{\prime} 3^{\prime \prime}$, etc., Fig. I 3 I, and draw the developed curve of intersection.

PROB. 20.- - To draw the orthographic projections of a cylindrical dome riveted to a cylindrical boiler of given dimensions.

Let the dimensions of the dome and boiler be.: dome. $26 \frac{1}{2} "$ diam. $\times 27^{\prime \prime}$ high, boiler $54^{\prime \prime}$ diam., plates $\frac{1}{2}$ " thick.

Apply to the solution of this problem the principles explained in Prob. No. I8, Fig. I3I.

When your drawings are completed, compare them with Figs. 133 and 134, which are the projections required in the problem.

Letter or number the drawing and be prepared to explain how the different projections were found.

Prob. 21.-To draw the development of the top gussetsheets of a locomotive wagon-top boiler of given dimensions.

First draw the longitudinal cross-section of the boiler to the dimensions given by Fig. I35, using the scale of $\mathrm{I}^{\prime \prime}=$ I $\mathrm{ft}$.

Then at any convenient point on your paper draw a straight line, and upon it lay off a distance $A B 35 \frac{1}{2}$ " long $=$ the straight part of the top of the gusset-sheet $G$, Fig. I35. With center $A$ and a radius $=27 \frac{78^{\prime \prime}}{}$ (the largest radius of the gusset) $+6^{\prime \prime}$ (the distance from the center of the boiler to the center of the gusset $C$, Fig. I35) $=33 \frac{7^{\prime \prime}}{}$, draw arc $\mathrm{I}$.

With center $B$ and a radius $=26 \frac{7}{\mathrm{~s}}$ (the smallest radius of the gusset) draw arc 2. Tangent to these arcs draw the 
straight line I, 2 extended, and through the points $A$ and draw lines I, $A$ and $2, B$ per. to I, 2.

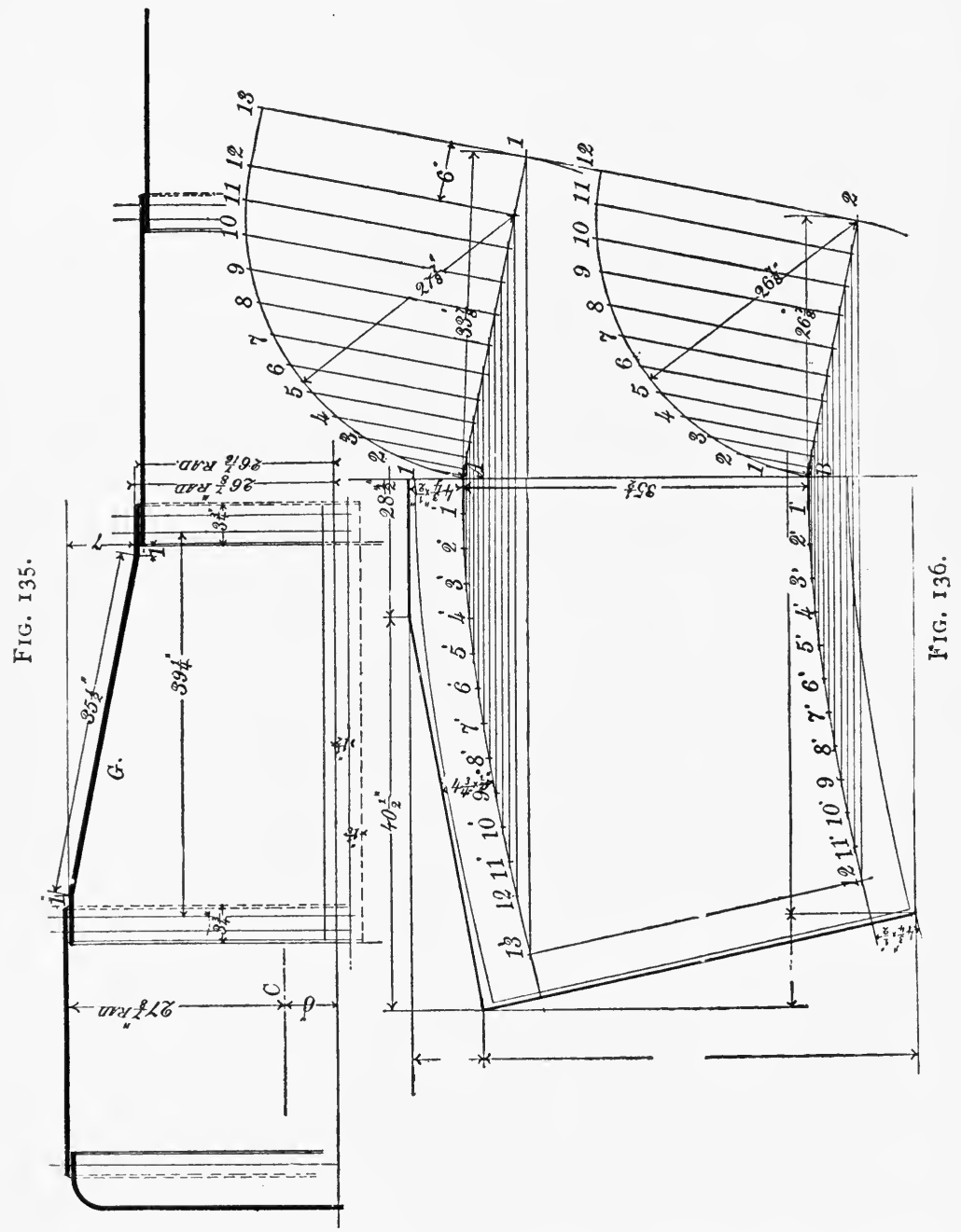

Take a point on the per. I, 2, $6^{\prime \prime}$ from the point $I$ as a center and through the point $A$ draw an arc with a radius $=27^{\frac{7}{8}}$. 
With point 2 as a center and $2 B$ as a radius $\left(26 \frac{7^{\prime \prime}}{8}\right)$ draw an arc through $B$ to meet the line $\mathbf{I}, 2$.

Divide both arcs into any number of parts, say 12, and through these divisions draw lines per. to and intersecting I $A$ and $2 B$ respectively. Through these intersections draw indefinite hors. and on these hors. step off the length of the arcs, with a distance $=$ one of the 12 divisions as follows:

On the first hors. lay off the length of the arc $A_{\mathrm{I}^{\prime}}$ and $B_{\mathrm{I}^{\prime}}$ $=A_{\mathrm{I}}$ and $B_{\mathrm{I}}$ respectively. Then from I' lay off the same distance to $2^{\prime}$ on the second hors. etc. Through these points draw curves $A 13^{\prime}$ and $B_{12}{ }^{\prime}$. Join points $12^{\prime}$ and $13^{\prime}$ with a straight line. Then $A B_{12}$, I 3 will be the developed half of the straight part of the gusset.

On the two ends or front and back of the gusset we have now to add $\mathrm{I}^{\prime \prime}$ for clearance $+3 \frac{\mathbf{8}^{\prime \prime}}{}$ for lap $+\frac{1}{2}^{\prime \prime}$ allowance for truing up the plates, total $=5 \frac{1^{\prime \prime}}{4}$. And to the sides $2 \frac{5^{\prime \prime}}{8}$ for lap $+\frac{1^{\prime \prime}}{2}$ allowance for truing up, total $=3 \frac{1}{8}{ }^{\prime \prime}$.

The outline of the developed sheet may now be drawn to include these dimensions with as little waste as possible, as shown by Fig. 136. Extreme accuracy is necessary in making this drawing, as the final dimensions must be found by measurement.

PROB. 22.-To draw the projections of a V-threaded screw and its nut of $3^{\prime \prime}$ diam. and $\frac{3^{\prime \prime}}{4}$ pitch.

Begin by drawing the center line $C, \mathrm{Fig}$. I37, and lay off on each side of it the radius of the screw $\mathrm{I}^{\frac{1}{2}}{ }^{\prime \prime}$. Draw $A B$ and $6 D$. Draw $A 6$ the bottom of the screw, and on $A B$ step off the pitch $=\frac{8}{4}{ }^{\prime \prime}$, beginning at the point $A$.

On line $6 D$ from the point 6 lay off a distance $=$ half the pitch $=\frac{3^{\prime \prime}}{8}$, because when the point of the thread has com- 
pleted half a revolution it will have risen perpendicularly a distance $=$ half the pitch, viz., $\frac{3^{\prime \prime}}{8}$.

Then from the point $6^{\prime \prime}$ on $6 D$ step off as many pitches as may be desired. From the points of the threads just found,

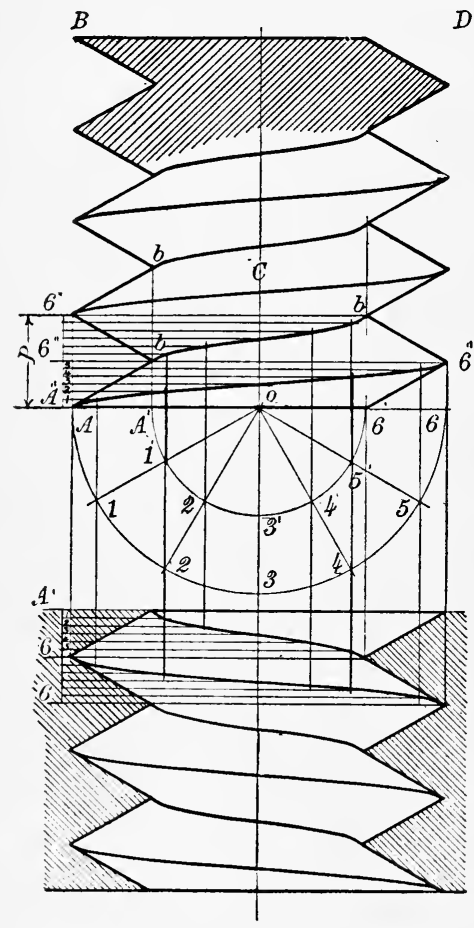

FIG. I37.

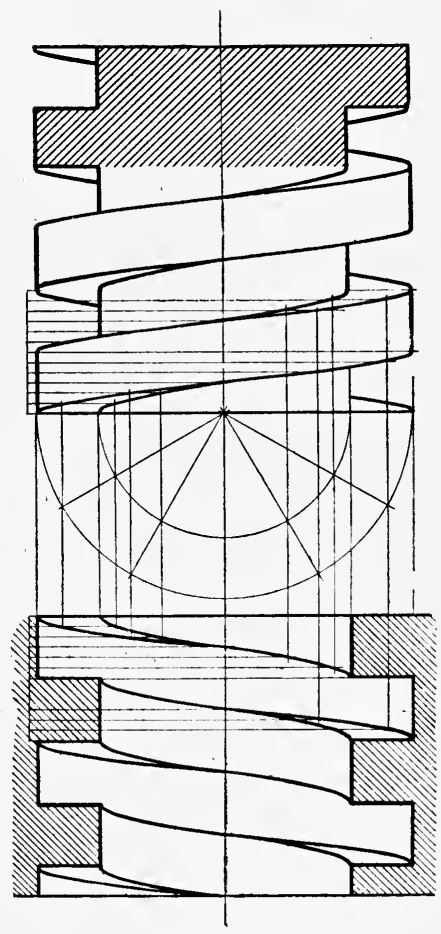

Fig. I3S.

draw with the $30^{\circ}$ triangle and $\mathrm{T}$-square the $\mathrm{V}$ of the threads intersecting at the points $b \ldots b$. the bottom of the threads.

At the point $O$ on line $A 6$ draw two semicircles with radii $\|$ the top and bottom of the thread respectively. Divide these into any number of equal parts and also the pitch $P$ into the same number of equal parts. Through these divisions draw hors. and pers. intersecting each other in the points as 
shown by Fig. 137, which shows an elevation partly in section and a section of a nut to fit the screw. Through the points of intersection draw the curves of the helices shown, using No. 3 of the "Sibley College Set" of Irregular Curves.

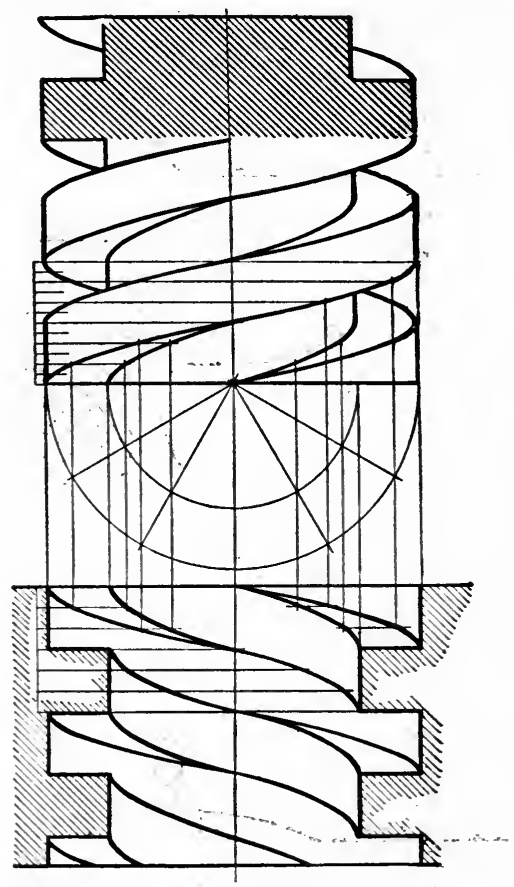

FIG. 139.

Prob. 22.-To draw the proj. of a șquare-threaded screw $3^{\prime \prime}$ diam. and $\mathrm{I}^{\prime \prime}$ pitch and also a section of its nut.

The method of construction is the same as for the last problem, and is illustrated by Fig. I 38 .

PRов. 22.-To draw the projections of a square double threaded screw of $3^{\prime \prime}$ diam. and $2^{\prime \prime}$ pitch, and also a section of its nut. 
The solution of this problem is shown by Fig. I 39, and further explanation should be unnecessary.

PROB. 23.-To draw the curve of intersection that is formed by a plane cutting an irregular surface of revolution.

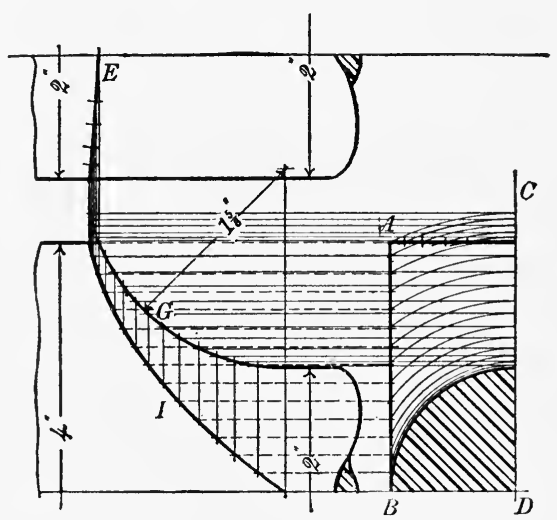

FIG. I40.

Figs. 140, I4I, and 142 show examples of engine connecting rod ends where the curve $I$ is formed by the inter-

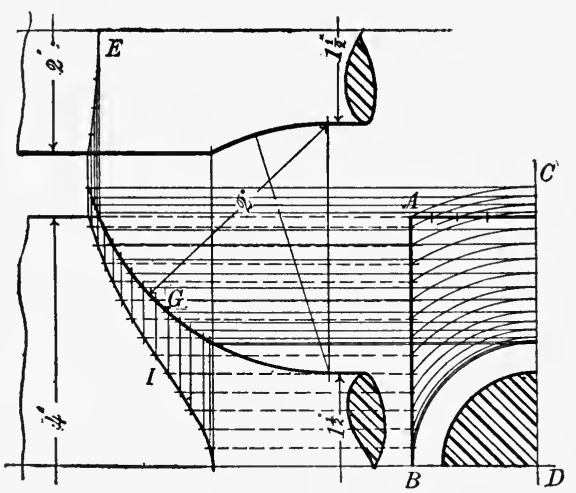

FIG. I4I.

section of the flat stub end with the surface of revolution of the turned part of the rod. 
The method of finding the curves of intersection are so plainly shown by the figures that a detailed explanation is deemed unnecessary.

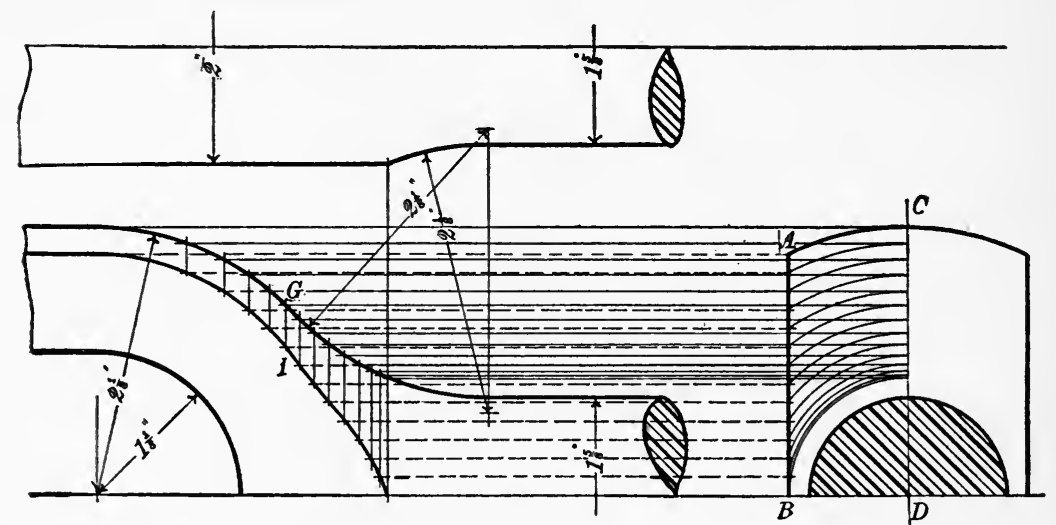

FIG. 142.

SHADE LINES, SHADES AND SHADOWS.

Shade Lines are quite generally used on engineering working drawings; they give a relieving appearance to the projecting parts, improve the looks of the drawing and make it easier to read, and are quickly and easily applied.

The Shading of the curved surfaces of machine parts is sometimes practiced on specially finished drawings, but on working drawings most employers will not allow shading because it takes too much time, and is not essential to a quick and correct reading of a drawing, especially if a system of shade lines is used.

Some of the principles of shade lines and shading are given below, with a few problems illustrating their commonest applications.

The shadows which opaque objects cast on the planes of 
projection or on other objects are seldom or never shown on a working drawing, and as the students in Sibley College are taught this subject in a course on Descriptive Geometry, it is omitted here.

\section{CONVENTIONS.}

The Source of Light is considered to be at an infinite distance from the object, therefore the Rays of Light will be represented by parallel lines.

The Source of Light is considered to be fixed, and the Point of Sight situated in front of the object and at an infinite distance from it, so that the Visual Rays are parallel to one another and per. to the plane of projection.

Shade Lines divide illuminated surfaces from dark surfaces.

Dark surfaces are not necessarily to be defined by those surfaces which are darkened by the shadow cast by another part of the object, but by reason of their location in relation to the rays of light.

It is the general practice to shade-line the different projections of an object as if each projection was in the same plane, e.g., suppose a cube, Fig. 143, situated in space in the third angle, the point of sight in front of it, and the direction of the rays of light coinciding with the diagonal of the cube, as shown by Fig. 144. Then the edges $a^{v} b^{v}, b^{v} c^{v}$ will be shade lines, because they are the edges which separate the illuminated faces (the faces upon which fall the rays of light) from the shaded faces, as shown by Fig. I44.

Now the source of light being fixed, let the point of sight remain in the same position, and conceive the object to be revolved through the angle of $90^{\circ}$ about a hor. axis so that a 
plan at the top of the object is shown above the elevation, and as the projected rays of light falling in the direction of the diagonal of a cube make angles of $45^{\circ}$ with the hor., then with the use of the $45^{\circ}$ triangle we can easily determine that the lower and right-hand edges of the plan as well as of the elevation should be shade lines.

This practice then will be followed in this work, viz.:

Shade lines shall be applied to all projections of an object,

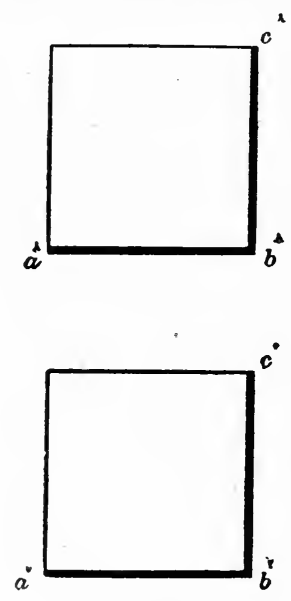

FIG. 143.

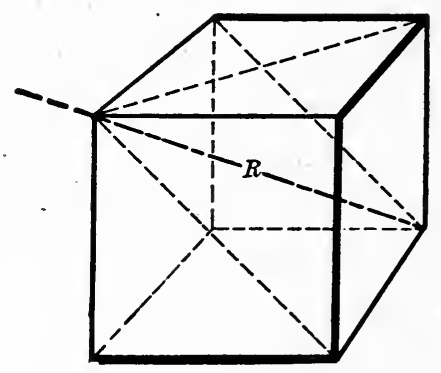

FIG. I44.

considering the rays of light to fall upon each of them, from the same direction.

Shade lines should have a width equal to 3 times that of the other outlines. Broken lines should never be shade lines.

The outlines of surfaces of revolution should not be shade lines. The shade-lined figures which follow will assist in illustrating the above principles; they should be studied until understood. 
SHADES.

The shade of an object is that part of the surface from which light is excluded by the object.

The iine of shade is the line separating the shaded from the illuminated part of an object, and is found where the rays of light are tangent to the object.

Brilliant Points.- "When a ray of light falls upon a surface which turns it from its course and gives it another direction, the ray is said to be reflected. The ray as it falls upon the surface is called the incident ray, and after it leaves the surface the reflected ray. The point at which the reflection takes places is called the point of incidence.

"It is ascertained by experiment-

"(a) That the plane of the incident and reflected rays is always normal to the surface at the point of incidence;

" (b) That at the point of incidence the incident and reflected rays make equal angles with the tangent plane or normal line to the surface.

"If therefore we suppose a single luminous point and the light emanating from it to fall upon any surface and to be reflected to the eye, the point at which the reflection takes place is called the brilliant point. The brilliant point of a surface is, then, the point at which a ray of light and a line drawn to the eye make equal angles with the tangent plane or normal line-the plane of the two lines being normal to the surface."

-Davies: Shades and Shadozes.

Considering the rays of light to be parallel and the point of sight at an infinite distance, the brilliant point on the surface of a splere is found as follows: Let $A^{v} C^{v}$ and $A^{h} C^{h}$. Fig. 
145, be a ray of light and $A^{v} A^{h}$ a visual ray. Bisect the angles contained between the ray of light and the visual ray as follows: Revolve $A^{v} C^{v}$ about the axis $A^{v}$ until it becomes parallel to the hor. plane at $A^{v} C_{1}^{v}$. At $C_{1}{ }^{v}$ erect a per. to intersect a hor. through $C^{h}$ at $C_{1}^{h}$ join $C_{1}{ }^{h} L^{h}(L$ may be any convenient

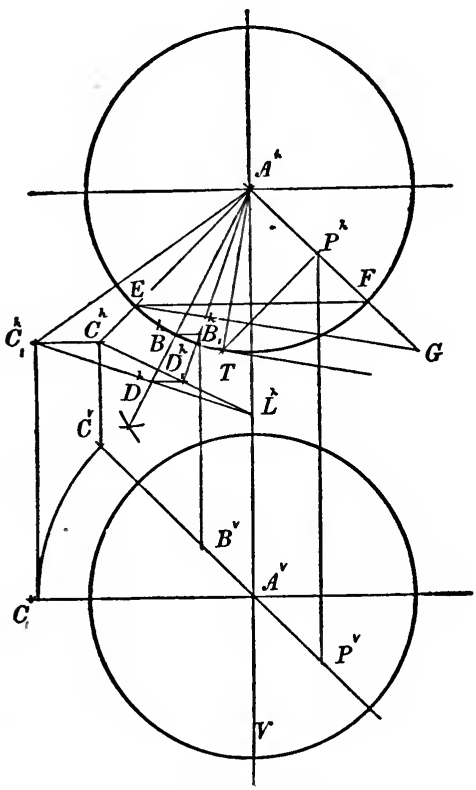

FIG. 145.

point on the line of vision), bisect the angle $L^{h} A^{h} C_{1}^{h}$ with the line $A^{h} D^{h}$. Join $C^{h} L^{h}$ and through the point $D^{h}$, draw a hor. cutting $C^{h} L^{h}$ at $D_{1}^{h}$, then $A^{h} D_{1}^{h}$ is the hor. projection of the bisecting line. A plane drawn per. to this bisecting line and tangent to 'the sphere touches the surface at the points $B^{v} B_{1}{ }^{h}$ where the bisecting lines pierce it. Therefore $B^{v} B^{h}$ are the two projections of the brilliant point. 
The point of shade can be found as follows:

Draw $A^{h} G$, Fig. I45, making an angle of $45^{\circ}$ with a hor. Join the points $E$ and $F$ with a straight line $E F$. Lay off on $A^{h} G$ a distance equal to $E F$, and join $E G$. Parallel to $E G$

FIG. I46.
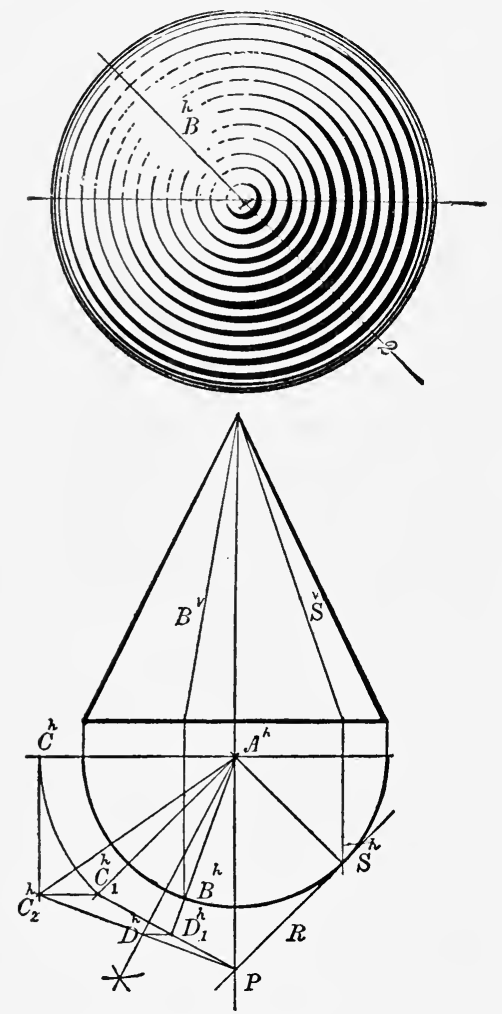

FIG. 148.
FIG. I47.

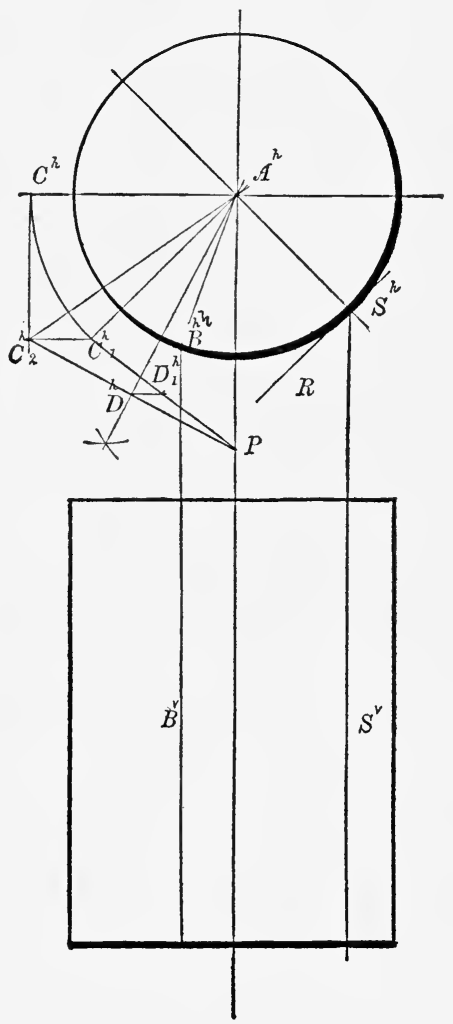

draw a tangent to the sphere at the point $T$. Through $T$ draw $T P^{h}$ per. to $A^{h} G$. From the point $P^{h}$ drop a per. to $P^{v}$. $P^{v}$ is the point of shade.

PROB. 24.-To shade the elevation of a sphere with graded arcs of circles. 
First find the brilliant point and the point of shade, and divide the radius 1,2 into a suitable number of equal parts, and draw arcs of circles as shown by Fig. I46, grading them by moving the center a short distance on each side of the center of the sphere on the line $B^{h} 2$ and varying the length of the radii to obtain a grade of line that will give a proper shade to the sphere. It is desirable to use a horn center to protect the center of the figure.

Fig. I49 shows the stippling method of shading the sphere.

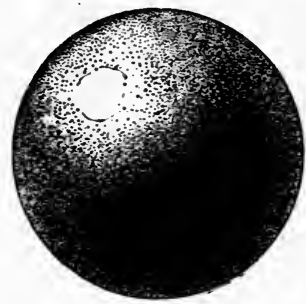

Fig. 149.

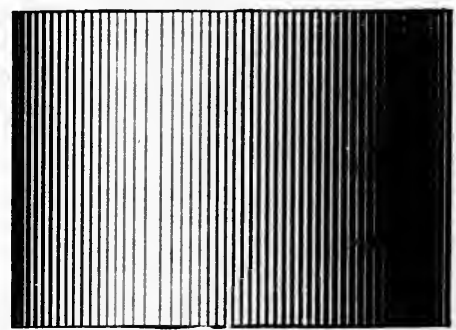

FIG. 150.

Prob. 25. - To shade a right cylinder with graded right lines.

Find the line of light $B^{o}$ by the same method used to find the brilliant point on the sphere, except that the line of light is projected from the point $B^{h}$ where the bisection line $A^{h} D$ cuts the circle of the cylinder.

The line of shade is found where a plane of rays is tangent to the cyl. at $S^{v}$ and $S^{h}$.

Fig. I 50 shows how the shading lines are graded from the line of shade to the line of light.

It will be noticed that the lines grow a little narrower to the right of the line of shade on Fig. 150; this shows where 
the reflection of the rays of light partly illumine the outline of the cylinder.

Prob. 26, Fig. I48. - To shade a right cone with graded right lines tapering toward the apex of the cone.

Find the elements of light and shade as shown by Fig. I48, and draw the shading-lines as shown by Fig. I5 I, grading their width toward the light and tapering them toward the apex of the cone.

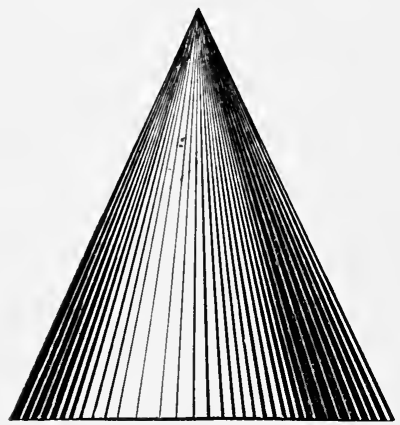

FIG. I5I.

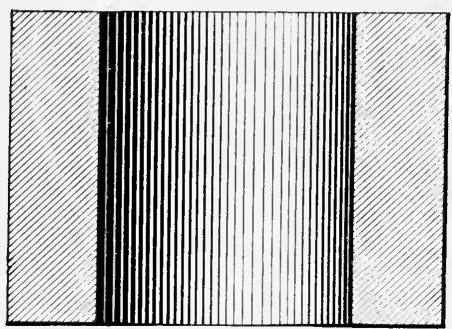

FIG. I52.

The mixed appearance of the lines near the apex of the cone on Fig. I 5 I can usually be avoided by letting each line dry before drawing another through it, or as some draftsmen do, stop the lines just before they touch.

Prob. 27.- To shade the concave surface of a section of a

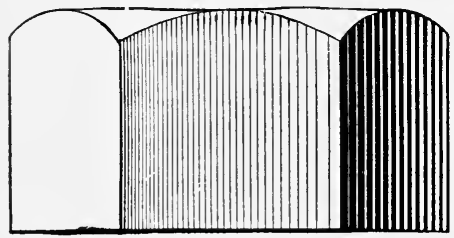

FIG. 153 . hollow cylinder.

Find the element of light. and grade the shading lines from it to both edges as shown by Fig. 152 .

Fig. I 53 shows a conventional method of shading a hexagonal nut. 


\section{SHADOWS.}

Let $R$, Fig. 154 , be the direction of the rays of light and $C$ an opaque body between the source of light and a

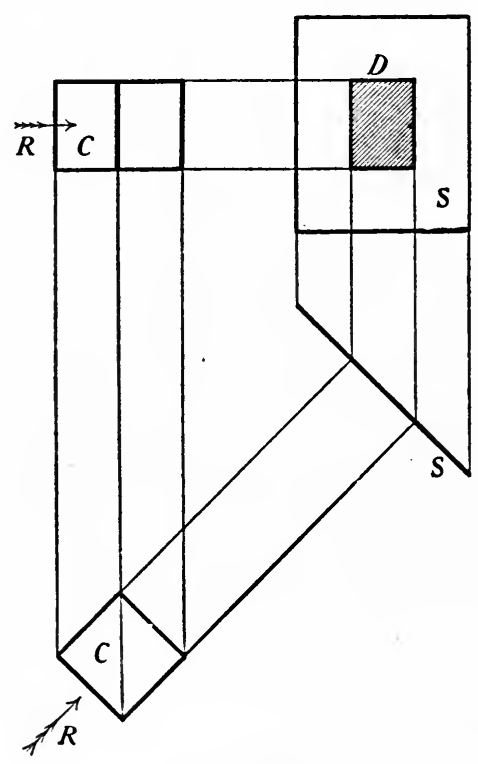

FIG. 154.

surface $S$. The body $C$ will prevent the rays from passing in that direction, and its outline will be projected at $D$ on the surface $S . \quad D$ is the shadow of $C$.

The line which divides the illuminated portion of the surface $S$ from the shadow $D$ is called the line of shadow.

Shadow of a Point. - If a line is drawn through a point in space in a direction opposite to the source of light, the point in which this line pierces the plane of projection is the shadow of the point on that plane. 
To find the shadow on the H.P. of a point in space in the first dihedral angle:

Let $A$, Fig. I55, be the point in space, and $R$ the direction of the ray of light; then $A_{1}{ }^{11}$ is the shadow of the point $A$ on H.P., and $A^{H} A_{1}{ }^{H}$ is the hor. proj. and $A^{l^{r}} A_{1}{ }^{{ }^{r}}$ the

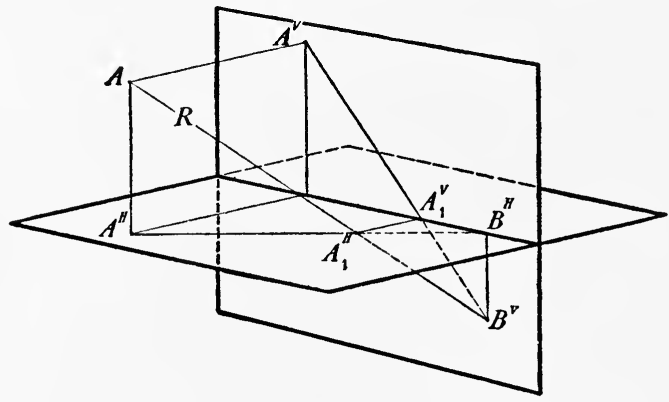

F1G. 155 .

vert. proj. of $R . B^{r}$ is the point where $R$ pierces $I^{r}$ when prolonged below H.P., and $B^{H}$ is its hor. proj. in the G.L. The projections of $R$ would then be $A^{l} B^{l}$ and $A^{H} B^{H}$.

The shadow of a point in $l$ may be found in a similar manner,

Shadou's of Right Lines. - The shadow of a right line on a plane may be determined by finding the shadows of two of its points and joining these by a right line; e.g., the shadow of the line $A B$, Fig. I 56 , on H.P. is found as follows:

Through the points $A^{l} B^{l^{V}}$ draw the rays $A^{l} A_{1}{ }^{r}$ and $B^{l} B_{1}{ }^{V}$ to intersect the plane of projection in G.L. in the points $A_{1}{ }^{r}$ and $B_{1}{ }^{r}$; from these points drop perpendiculars to meet rays drawn through $A^{I I}$ and $B^{H}$ in the points $A_{1}{ }^{H}$ and $B_{1}{ }^{H}$. A line drawn from $A_{1}{ }^{1 I}$ to $B_{1}{ }^{H I}$ is the shadow of $A B$ on H.P.

If a righ tline is parallel to the plane of projection its shadow will be parallel to the line itself. 
If a line coincides with a ray of light, its shadow on any surface will be a point.

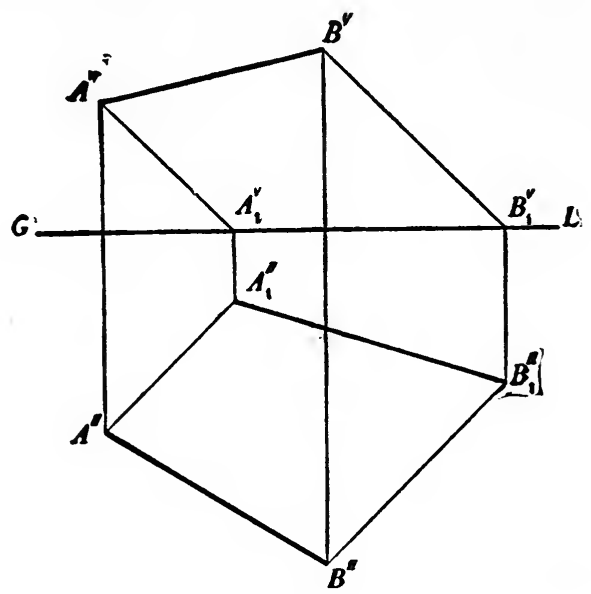

FIG. 156.

Рвов. 28.-To find the shadow of a right line on V.P. and H.P.

Let $A B$, Fig. 157 , be the given line. Find the shadows

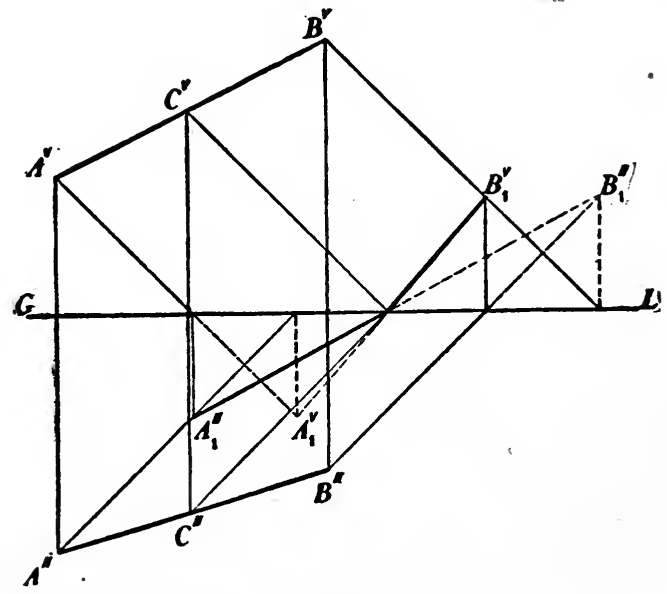

FIG. 157. 
of the points $A$ and $B$ by passing rays through each of their projections to make angles of $45^{\circ}$ with G.L. The shadow of $A^{H}$ on H.P. is found at $A_{1}{ }^{H}$, and that of $B^{H}$ at $B_{1}{ }^{H}$, where the rays through these points intersect the H.P. The shadow of $A^{V}$ on V.P. is found at $A_{1}{ }^{V}$ and that of $B^{V}$ at $B_{1}{ }^{v}$, where the rays through these points intersect V.P. Join $A_{1}{ }^{H}$ and $B_{1}{ }^{H}$ with a straight line and we have the shadow of $A B$ on H.P., and the shadow on V.P. is found in the same way by joining with a straight line the points $A_{1}{ }^{V}$ and $B_{1}{ }^{V}$.

That part of the shadow which falls on V.P. below G.L., and on H.P. above G.L., is called the secondary shadow, because it makes a second intersection, i.e., it is conceived to have passed through V.P. and made an intersection with H.P. behind V.P. With the use of the secondary shadow problems like this are easier of solution.

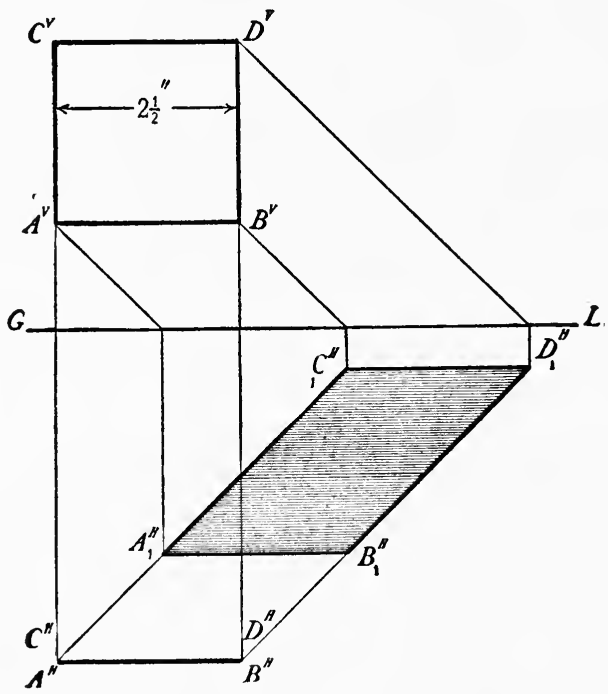

FIG. I58. 
PROB. 29.-ABCD, Fig. $15^{8}$, is a square plate parallel to V.P. ; find its shadow on H.P.

Through the points $A^{V}, B^{V}, D^{V}$, and $A^{H} C^{H}, B^{H} D^{H}$, draw rays making the angle of $45^{\circ}$ (or any other angle which may be adopted) with G.L., and determine the shadows of these points as explained in Fig. 155 . They will be found in the points $A_{1}{ }^{H} B_{1}{ }^{H}, C_{1}{ }^{H}, D_{1}{ }^{H}$. Join these points with right lines and they will form the line of shadow of the square plate on H.P.

Pвов. 30.-To find the shadow of a cube on V.P. with one face in V.P. and the other faces parallel or perpendicular to H.P.

Fig. I 59 shows the cube in the given position. The line

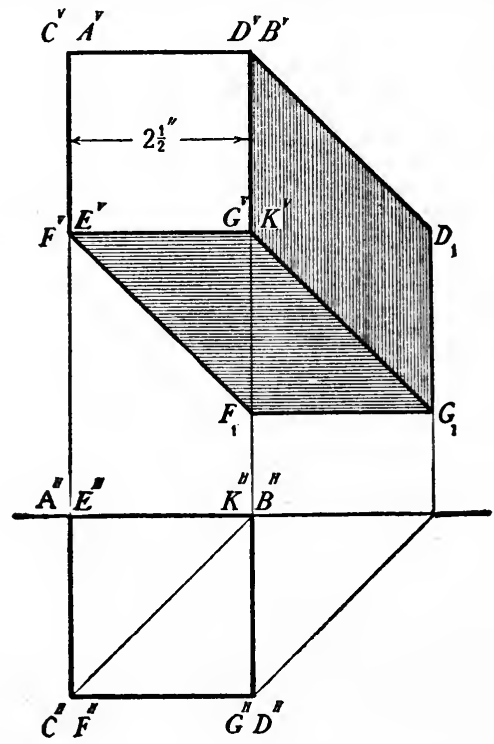

FIG. I59.

of shade is composed of edges $E F, F G, G D, D B$, and the edges $A E$ and $A B$ in V.P. which coincide with their shadows. 
The shadow of $E F$ is $E^{r} F_{1}$, of $F G$ is $F_{1} G_{1}$, of $G D$ is $G_{1} D_{1}$, of $D B$ is $D_{1} B^{r}$. The shadows of the edges $A E$ and $A B$ coincide with the lines. These shadows are found by the same rules used for finding the shadows of a line in Prob. 28. The line of shadow is $B^{r} D_{1} G_{1} F_{1} F^{r} E^{r} A^{r} D^{r}$. The visible line of shadow is $B^{V} D_{1} G_{1} F_{1} E^{r} C^{r} D^{V}$.

PROB. 31.-To find the shadow of a rectangular abacus on the face of a rectangular pillar.

Assume the hor. and vert. projs. of the abacus and pillar to be as shown in Fig. 160.

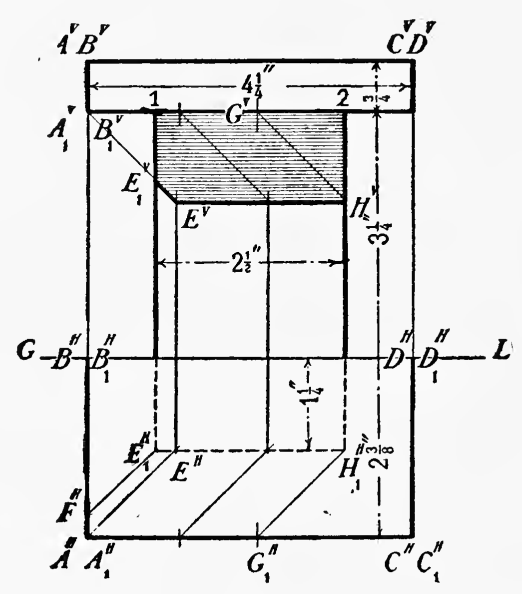

FIG. 160 .

The line of shade of the abacus is seen to be the edges $A_{2}{ }^{H} B_{1}{ }^{H}$ and $A_{1}{ }^{H} C_{1}{ }^{H}$. The plane of rays through edge $A_{1}{ }^{H} B_{1}{ }^{H}$ is per. to V.P., and the line $A_{1}{ }^{V} E^{l}$ is its vert. proj. or trace ; its hor. trace is $A_{1}{ }^{H} E^{I}$. The shadow on the left side face, is vertically projected in the point $E_{1}{ }^{V}$ where the plane of rays intersects that face. The ray through the point $A_{1}{ }^{I I}$ pierces the front face in the point $E^{H}$, which is the shadow of $A_{1}{ }^{I}$, 
and $E_{1}{ }^{H} E^{H}, E_{1}{ }^{V} e^{V}$ is the shadow of the part $F^{H} A_{1}{ }^{H}$ on this face.

The line $A_{1}{ }^{H} C_{1}{ }^{H}$ is parallel to the front face, therefore its shadow on it will be parallel to itself and pass through $E$.

The visible line of shadow is now found to be $E_{1}^{V} E^{v} H^{V}{ }_{2}$.

PROB. 32.-Construct the shade of an upright liex. prism and its shadoa on both planes.

Fig. I6I shows the given prism with its line of shade

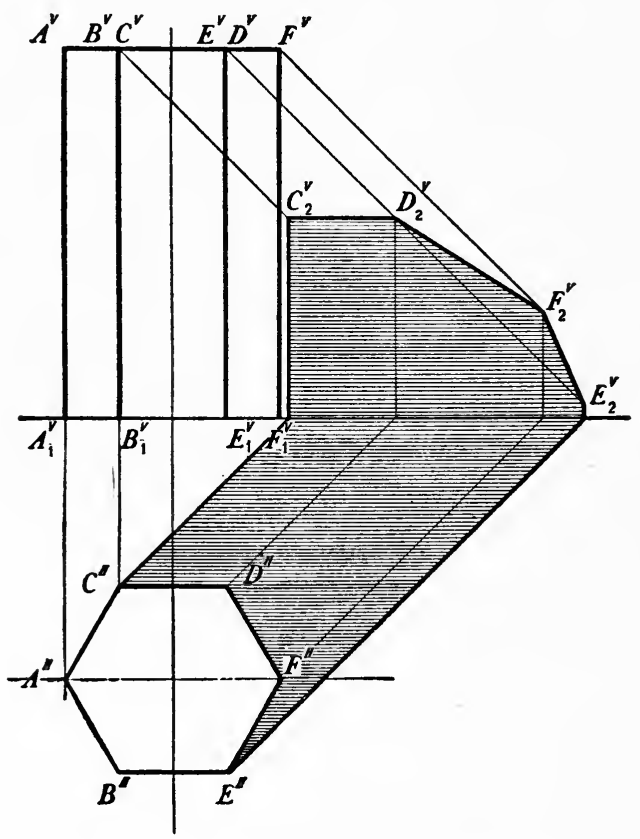

FIG I6I.

$A_{1}{ }^{V} B_{1}^{V} E_{1}^{V} D^{V} F^{V}$ on the vert. proj., $C^{H} D^{H} F^{H} E^{H}$ on the hor. proj., and its shadow on both planes.

PROB. 33.-Given a circular plate parallel to one coordinate plane; construct its shadow on the other plane. 
Let $A^{V} B^{V} C^{V} D^{V}$ and $A^{H} C^{H}$, Fig. 162, be the projections of the circular piate.

Circumscribe a square $E^{V} G^{V}$ about the circle; its shadow on H.P. will be the parallelogram $A^{I I} G^{H}$, and the shadows of the points $A^{v} B^{v} C^{v} D^{V}$ are projected in the points

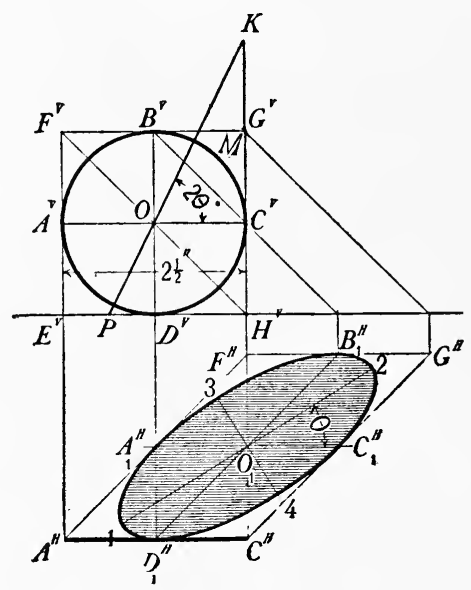

FIG. I62.

$A_{1}{ }^{H} B_{1}{ }^{H} C_{1}{ }^{I I} D_{1}{ }^{I I}$. The shadow of the inscribed circle is an ellipse tangent to the parallelogram at the points $A_{1}{ }^{I I} B_{1}{ }^{H} C_{1}{ }^{H} D_{1}{ }^{I I}$, with $B_{1}{ }^{I I} D_{1}^{H}$ and $A_{1}{ }^{I I} C_{1}{ }^{I I}$ as conjugate diameters.

The position and length of the axes of the ellipse of shadow may be found as follows:

Erect a perpendicular at the point $C^{r^{*}}$ making $G^{r} K^{r^{*}}$ equal to radius of the circle draw $K O P$; then $K P$ is equal to the major and $M K$ to the minor axis, and angle $\theta$ is twice the angle of the transverse axis with the horizontal conjugate diam.; i.e., $K P$ is equal to I, $2, N K$ to 3,4 , and $2, O_{1} C_{1}{ }^{I I}$, or angle $\theta$, is equal to half $K O C^{V}$. 
PRов. 34.-Find the shade of a cylindrical column and abacus, and the shadow of the abacus on the column.

Let $A^{V} B^{V} C^{V}$ and $A^{H} B^{H} C^{H}$, Fig. 163, be the projections of the abacus, $D^{H} E^{H} F^{H}$ and $D^{H} D^{V} G^{V} F^{H}$ the projections of the column.

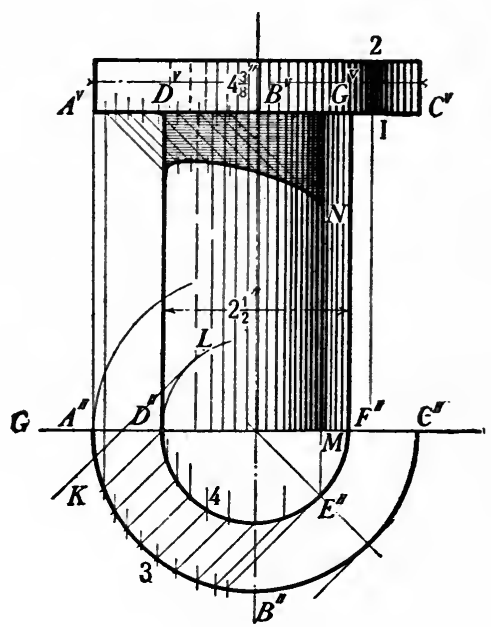

Fig. I63.

The line of shade on the column is found by passing two planes of rays tangent to the column perpendicular to H.P. and parallel to the hor. proj. of the ray of light. $K L$ and $E^{H}$ are the traces of these planes tangent to the column at the points $L$, and $E^{H}$ and $M N$ the visible line of deepest shade on the cylindrical column.

The deepest line of shade I, 2 on the abacus is found in the same way.

The line of shadow on the column of that portion of the lower circumference of the abacus which is toward the source of light is found by passing vertical planes of rays, as 3,4 , to 
determine any number of points in the line, and joining these points by a line as shown in Fig. 163 .

Prob. 35.-Find the shade of an oblique cone and its shadowe on H.P.

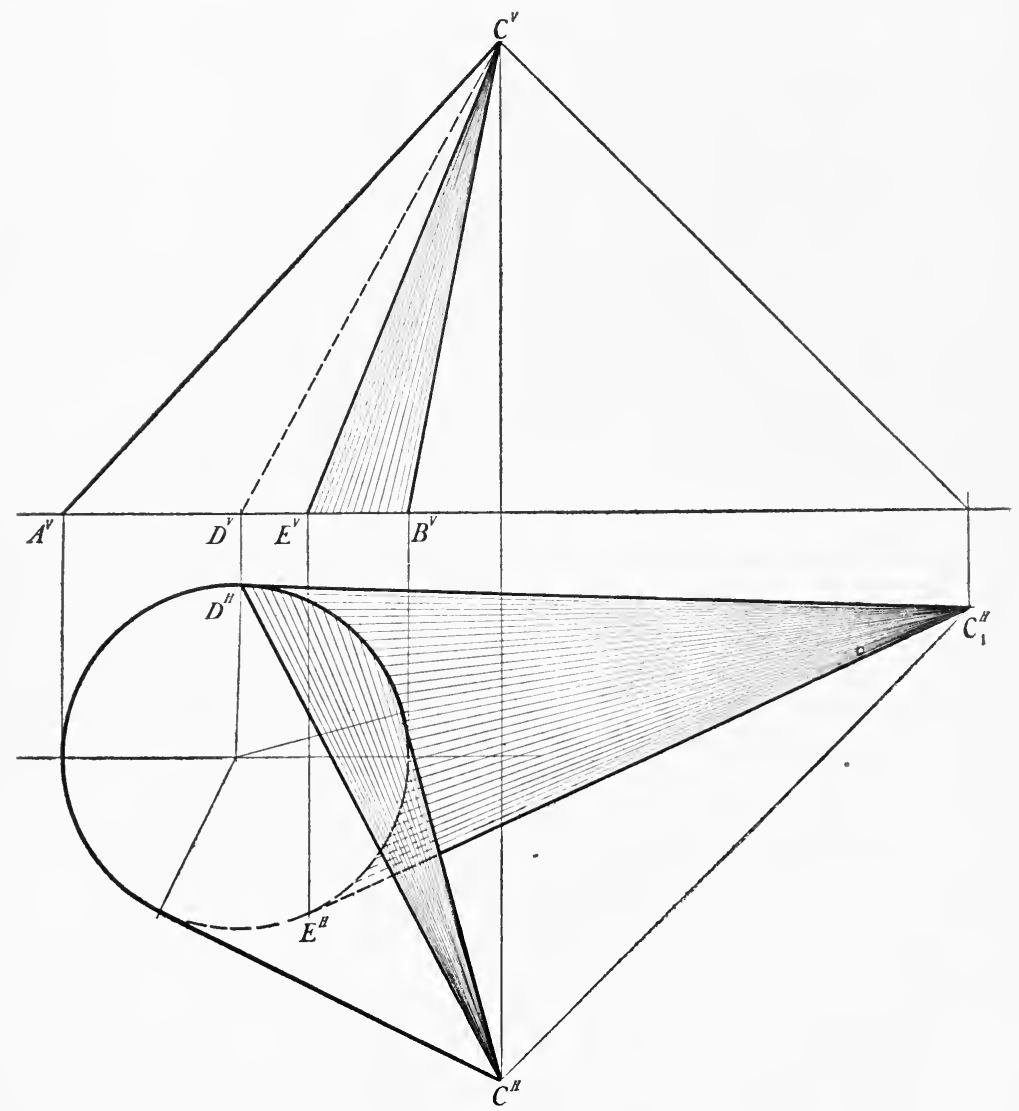

FIG. I64.

Take the cone as given in Fig. I64. Pass two planes of rays tangent to the cone; their elements of contact will be the deepest lines of shade. To determine the elements of contact draw a ray through $C^{V} ; C_{1}{ }^{H}$ is its hor. trace. From 
$C_{1}{ }^{H}$ draw lines tangent to the base at $D$ and $E$; the lines of contact are $C E$ and $C D$, and $E C D$ is the line of shade.

The visible line of shade on H.P. is $E^{H} D^{H}$, and on V.P. it is $C^{V} E^{V}$. The shadow on H.P. is $E^{H} C_{1}^{H} D^{H}$.

PROB. 36.- To draw a front and end elevation of a rectangular hollow box with a rectangular block on each face, each block to have a rectangular opening, and all to be properly shade-lined and drawn to the dimensions given on Fig. 165 .

Draw the hor. center line first, and then the vertical center line of the end view. About these center lines on the end el-

FIG. I65.

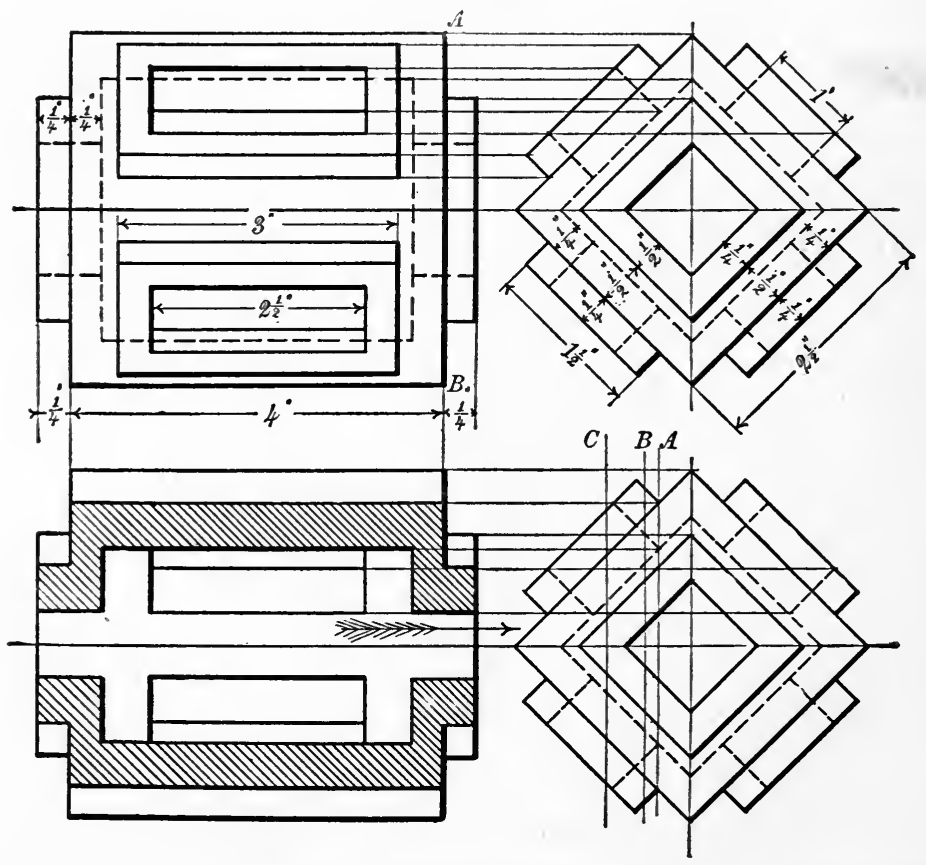

Fig. 166.

evation construct the squares shown and erect the edges of the blocks. Next draw the hidden lines indicating the thickness 
of the walls of the box and the openings through the blocks, measuring the sizes carefully to the given dimensions.

Draw the front elevation by projecting lines from the various points on the end elevation, and assuming the position of the line $A B$ measure off the lengths of the hor. lines and erect their vert. boundaries as shown by the figure.

Prob. 37.-Given the end elevation of the last prob., cut by three planes $A, B$ and $C$, Fig. 166. Draw the projections of these sections when the part to the left of the cutting plane has been removed, and what remains is viewed in the direction of the arrow, remembering that all the visual rays are parallel.

These drawings and all that may follow are to be properly shade-lined in accordance with the principles given above.

\section{ISOMETRICAL DRAWING.}

In orthographic projection it is necessary to a correct understanding of an object to have at least two views, a front and end elevation, or an elevation and plan, and sometimes even three views are required.

Isometric drawing on the other hand shows an object completely with only one view. It is a very convenient system for the workshop. Davidson in his Projection calls it the "Perspective of the Workshop." It is more useful than perspective for a working drawing, because, as its name implies (" equal measures") it can be made to any scale and measured like an orthographic drawing. It is, however, mainly employed to represent small objects, or large objects drawn to a small scale, whose main lines are at right angles to each other.

The principles of isometrical drawing are founded on a cube resting on its lower front corner, I, Fig. 167, and its base 
elevated so that its diagonal $A B$ is parallel to the horizontal plane. Then if the cube is rotated on the corner I until the diagonal $A B$ is at right angles to the vert. plane, i.e., through an angle of $90^{\circ}$, the front elevation will appear as shown at 1, 2, 3, 4, Fig. 167, a regular hexagon.

Now we know that in a regular hexagon, as shown by Fig. 167, the lines I $A, A_{3}$, etc., are all equal, and are easily drawn

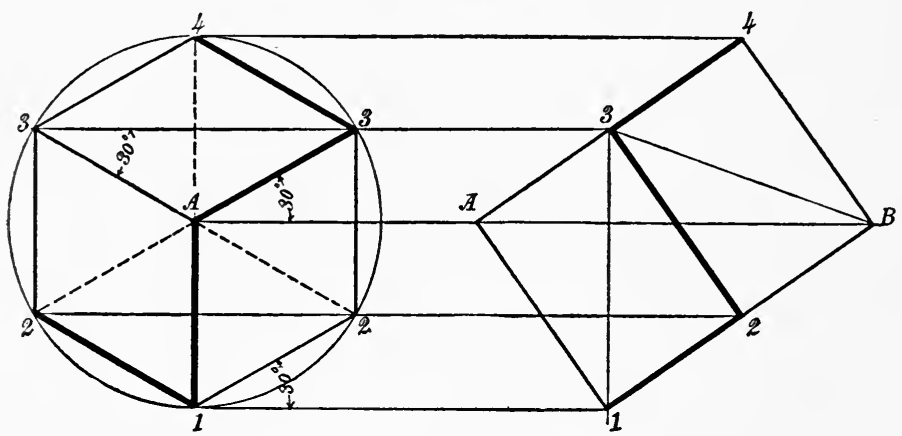

FIG. I67.

with the $30^{\circ} \times 60^{\circ}$ triangle. But although these lines and faces appear to be equal, yet, being inclined to the plane of projection, they are shorter than they would actually be on the cube itself. However, since they all bear the same proportion to the original sizes, they can all be measured with the same scale.

We will now describe the method of making an isometrical scale.

Draw the half of a square with sides $=2 \frac{1}{2}{ }^{\prime \prime}$, Fig. $\mathbf{I} 68$. These two sides will make the angle of $45^{\circ}$ with the horizontal. Now the sides of the corresponding isometrical square, we have seen, make the angle of $30^{\circ}$ with the horizontal, so we will 
draw I 4,34 , making angles of $30^{\circ}$ with 1,3 . The difference then between the angle 2, I, 3 and the angle $4,1,3$ is $15^{\circ}$, and the proportion of the isometrical projection to the actual object is as the length of the line 3,2 to the line 3,4 . And if the line 3,2 be divided into any number of equal parts, and lines be drawn through these divisions par. to 2, 4 to cut the line 3, 4 in corresponding divisions, these will divide 3,4 proportionately to 3,2 .

Now if the divisions on 3,2 be taken to represent feet and those on 3, 4 to represent 2 feet, then 3,4 would be an isometrical scale of $\frac{1}{2}$.

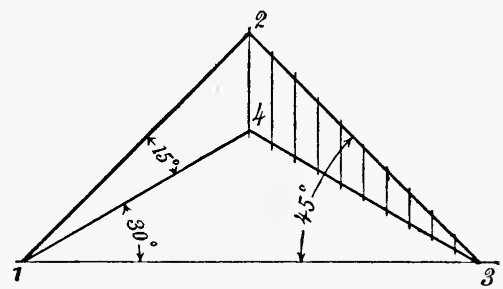

FIG. 168.

Since isometrical drawings may be made to any scale, we may make the isometrical lines of the object $=$ their true size. This is a common practice and precludes the need of a special isometrical scale.

The Direction of the Rays of Light. - The projection of a ray of light in isometrical drawing will make the angle of $30^{\circ}$ with the horizontal as shown by the line 3,2 on the front elevation of the hex., Fig. 167. And the shade lines will be applied as in ordinary projection.

Prob. 38. - To make the isometrical drawing of a twoarmed cross standing on a square pedestal. 
Begin by drawing a center line $A B$, Fig. 169, and from the point $A$ draw $A C$ and $A D$, making an angle of $30^{\circ}$ with the horizontal. Measure from $A$ on the center line $A B$ a distance $=\frac{5}{16}{ }^{\prime \prime}$, and draw lines par. to $A C, A D$; make $A C$ and $A D 2 \frac{5}{8} "$ long and erect a perpendicular at $D$ and $C$, completing the two front sides of the base, etc.

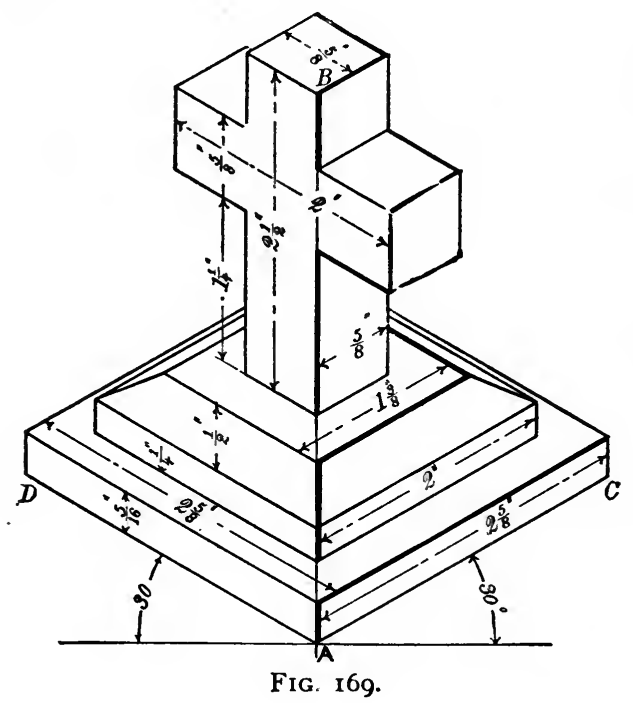

PROB. 39.-To make the isometrical drawing of a hollow cube, with square block on each face and a square hole through each block, to dimensions given on Fig. I 70.

As before, first draw a center line, and make an isometrical drawing of a $2 \frac{1}{2}$ " cube, and upon each face of it build the blocks with the square holes in them, exactly as shown in Fig. I 70 .

Prob. 40.-To project an isometrical circle.

The circle is enclosed in a square, as shown by Fig. I 7 I. 
Draw the circle with a radius $=2^{\prime \prime}$ and describe the square I, 2, 3, 4 about it.

Draw the diagonals $\mathrm{I}, 2,3,4$ and the diameters $5,6,7,8$ at right angles to each other.

Now from the points I and 2 draw lines I $A$, I $B$ and $2 A$, $2 B$, making angles of $30^{\circ}$ with the hor. diagonal I, 2. And

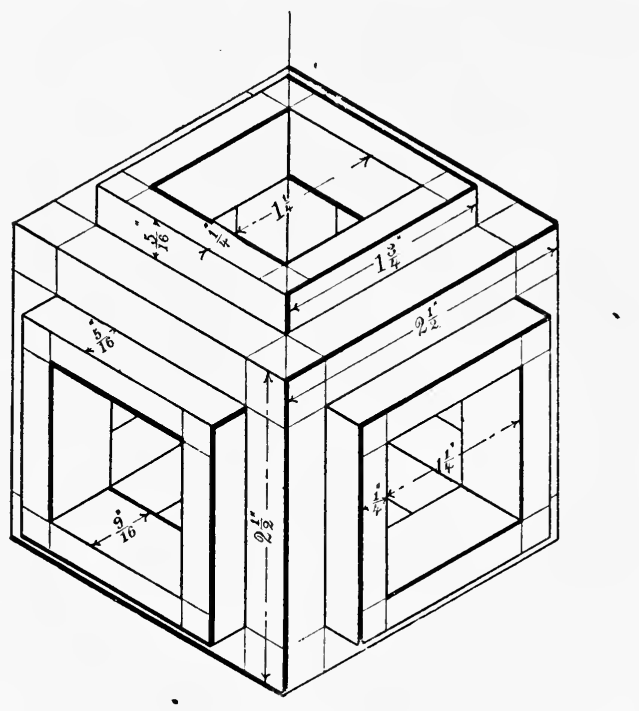

FIG. I70.

through the center $O$ draw $C D$ and $E F$ at right angles to the isometrical square.

The points $C \cdot D, E F$, and $G H$ will be points in the curve of the projected isometrical circle, which will be an ellipse.

The ellipse may be drawn sufficiently accurate as follows:

With center $B$ and radius $B C$ describe the arc $C F$ and extend it a little beyond the points $C$ and $F$, and with center $A$ and same rad. describe a similar arc, then with a rad. which 


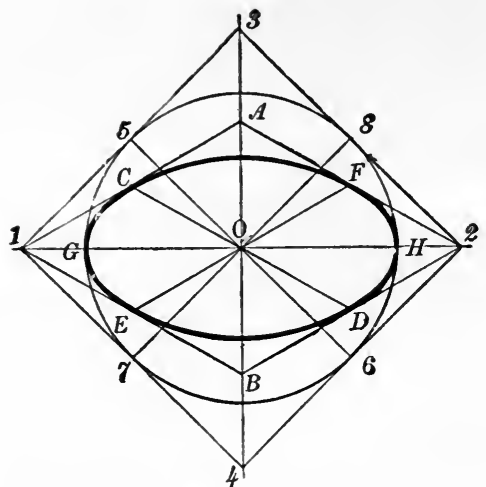

Fig. 17 1.

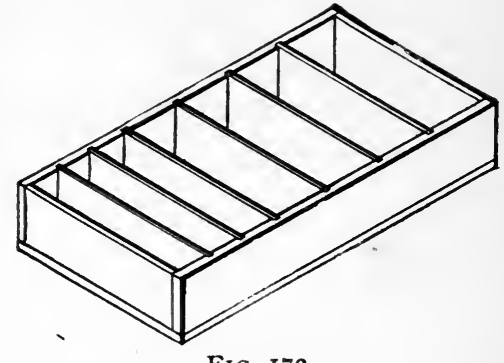

FiG. 172.

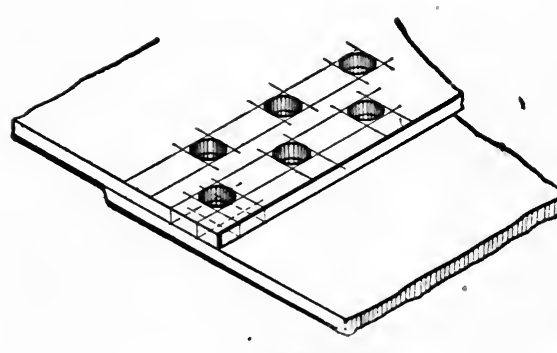

FIG. 173 .
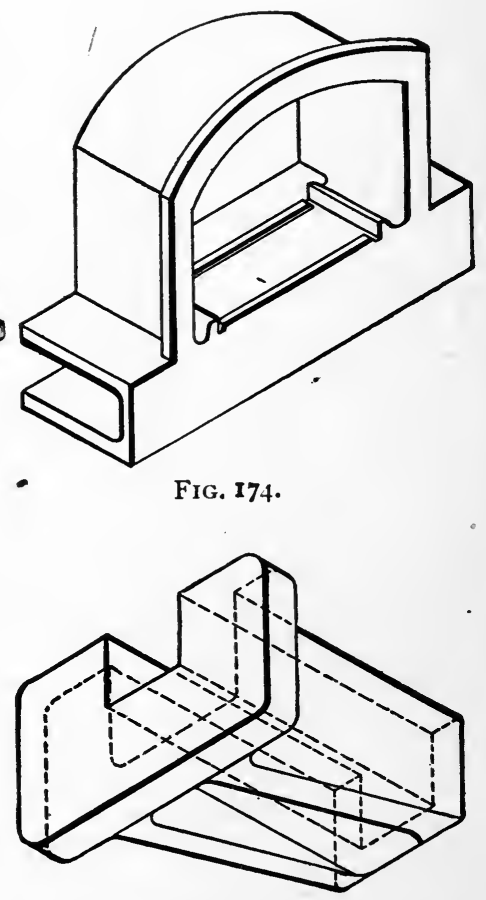

FIG. 174.

Fig. 176 .
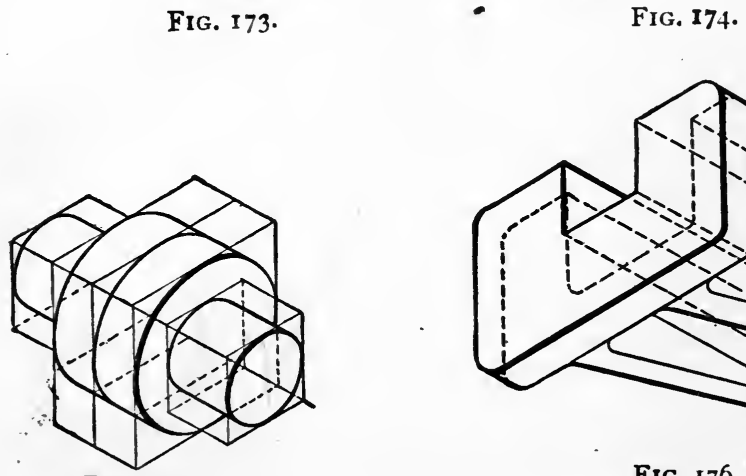

FiG. 175 . 
may readily be found by trial, draw arcs through the points $G$ and $H$ and tangent to the two arcs already described.

Figs. 172, 173, I74, I75, I76, and 177 are for practice in the application of the preceding principles, and at least one of these should be drawn, or it would be better still if the stu-

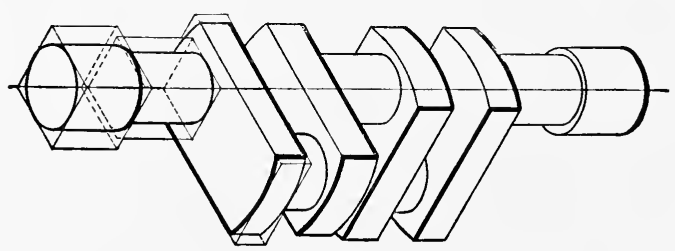

FIG. I77.

dent would attempt to make an isometrical projection of his instrument-box, desk, or any familiar object at hand. These figures may be measured with the $I_{2}{ }^{\prime \prime}$ scale and drawn with the $2^{\prime \prime}$ scale.

\section{WORKING DRAWINGS.}

Working drawings are sometimes made on brown detailpaper in pencil, traced on tracing-paper or cloth, and then blue printed.

The latter process is accomplished as follows:

The tracing is placed face down on the glass in the printing-frame, and the prepared paper is placed behind it, with the sensitized surface in contact with the back of the tracing.

In printing from a negative the sensitized surface of the prepared paper is placed in contact with the film side of the negative, and the face is exposed to the light.

The blue-print system for working drawings has many drawbacks, e.g., the sectioned parts of the drawing require to 
be hatch-lined, using the standard conventions already re ferred to for the different materials. This takes a great deal of time. The print has usually to be mounted on cardboard, although this is not always done, and unless it is varnished the frequent handling with dirty, oily fingers soon makes it unfit for use.

Changes can be made on the prints with soda-water, it is true, but they seldom look well, and when many changes or additions require to be made it is best to make them on the tracing and take a new print. And the sunlight is not always favorable to quick printing. So taking everything into consideration the system of making working drawings directly on cards and varnishing them is probably the best. It is the system used by the Schenectady Locomotive Works and many other large engineering establishments. In size the cards are made $9^{\prime \prime} \times 12^{\prime \prime}, 12^{\prime \prime} \times 18^{\prime \prime}, 18^{\prime \prime} \times 24^{\prime \prime}$; they are made of thick pasteboard mounted with Irish linen recordpaper. The drawings are pencilled and inked on these cards in the usual way, and the sections are tinted with the conventional colors, which are much quicker applied than hatchlines. The face of the drawing is protected with two coats of white shellac varnish, while the back of the card is usually given a coat of orange shellac.

The white varnish can easily be removed with a little alcohol, and changes made on the drawing, and when revarnished it is again ready for the shop.

We will now try to describe what a working drawing is and what it is for. In the hands of an experienced workman a working drawing is intended to convey to him all the necessary information as to shape, size, material, and finish to 


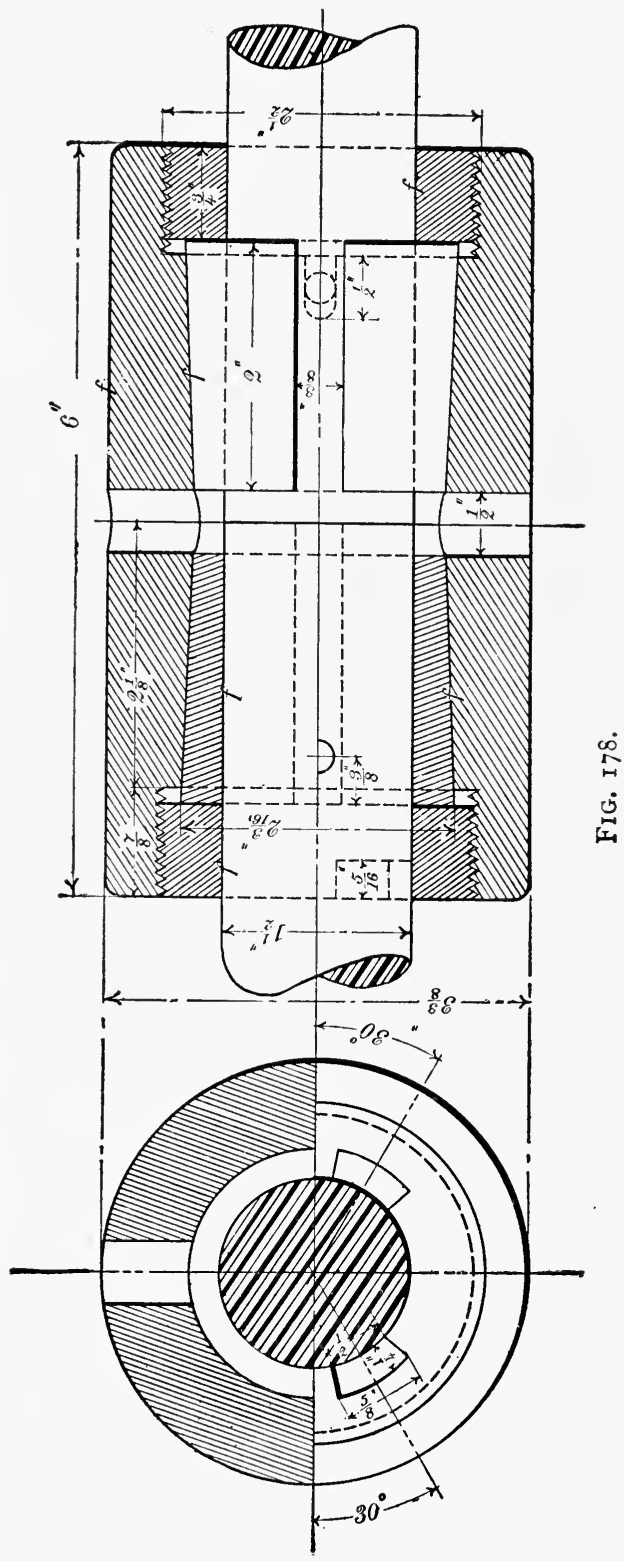



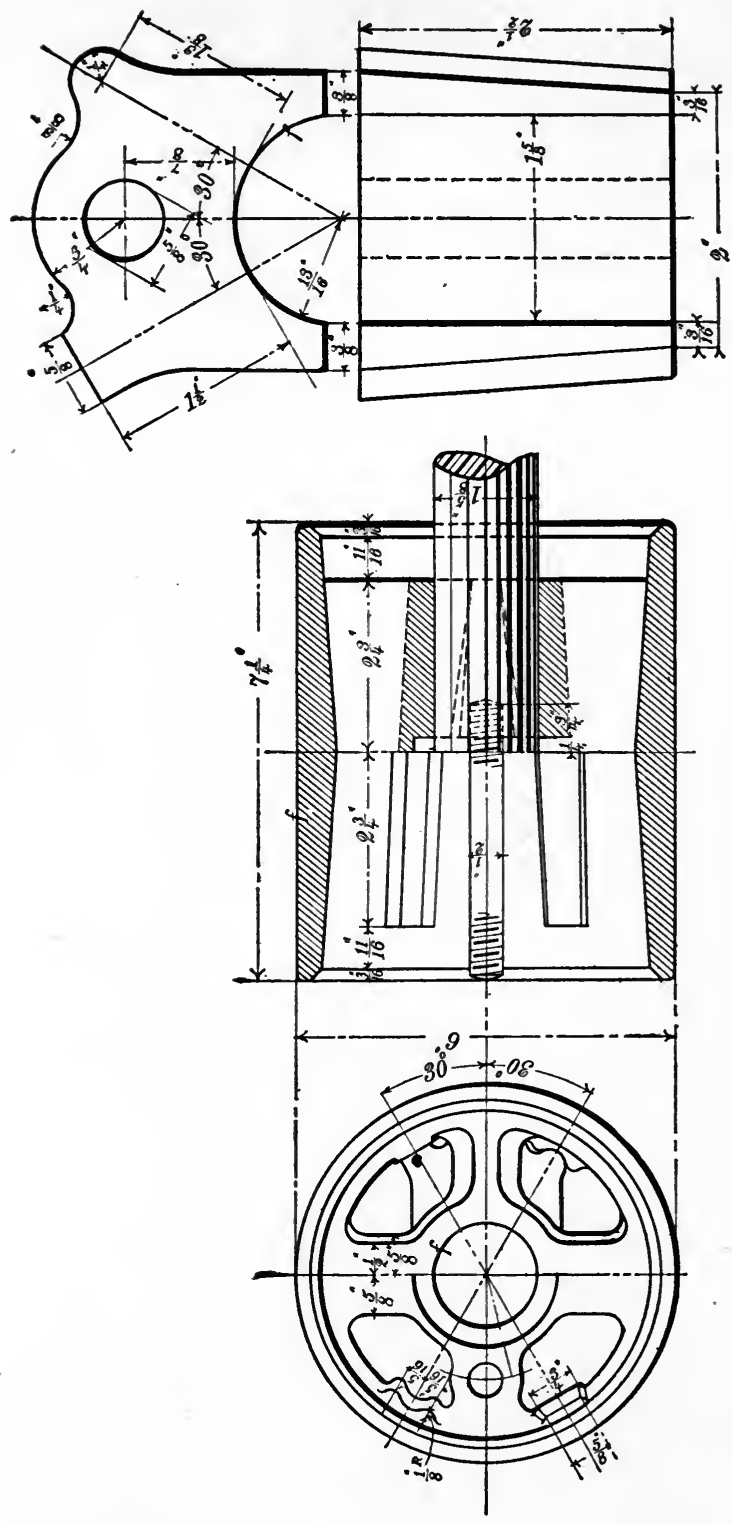

亩 
enable him to properly construct it without any additional instructions. This means that it must have a sufficient number of elevations, sections, and plans to thoroughly explain and describe the object in every particular. And these views should be completely and conveniently dimensioned. The dimensions on the drawing must of course give the sizes to which the object is to be made, without reference to the scale to which it may be drawn. The title of a working drawing should be as brief as possible, and not very large-a neat, plain, free-hand printed letter is best for this purpose.

Finished parts are usually indicated by the letter " $f$," and if it is all to be finished, then below the title it is customary to write or print " finished all over."

The number of the drawing may be placed at the upper left-hand corner, and the initials of the draftsman immediately below it.

A second-year course, entitled Mechanical Drawing and Elementary Machine Design, is in preparation, and will shortly be published:

Figs. 178 and 179 show working drawings of two shaftcouplings, fully figured, sectioned, and shade-lined. 


\section{INDEX.}

A

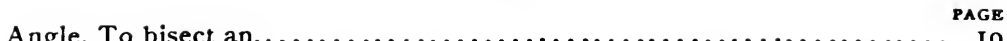

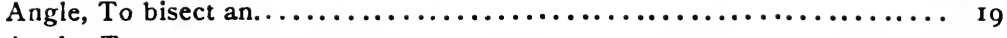

Angle, To construct an .................................. r $_{5}$

Anti-friction curve, "Schiele's"..$\ldots \ldots \ldots \ldots \ldots \ldots \ldots \ldots \ldots \ldots \ldots \ldots \ldots$. 50

A rched window-opening, To draw an..................... 53

Arkansas oil-stones.................................. 5

B

Baluster, To draw a................................. 53

Board, Drawing............................

Bow instruments.................................. 2

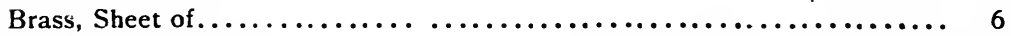

Breaks, Conventional............................... $6 \mathbf{r}$

Brilliant points................................. 106

C

Celluloid, Sheet of thin.................................... 5

Center lines..................................... 60

Cinquefoil ornament, To draw the....................... 53

Circle, Arc of a. To find the center of an.................... 32

Circle, Arc of a, To draw a line tangent to an.................. 33

Circle, To draw a right line equal to half the circumference of a........ $3 \mathbf{I}$

Circle, To draw a tangent between two................... 33

Circle, To draw tangents to two........................ 34

Circle, To draw an arc of a, tangent to two straight lines........... 34

Circle, To inscribe a, within a triangle................... 35

Circle, To draw an arc of a, tangent to two circles.............. 36

Circle, To draw an arc of a, tangent to a straight line and a circle...... 37

Circle, To construct the involute of a...................... 45

Circle, To find the length of an arc of a, approximately. .......... 47 
49

Compass...................................... 2

Conventions..................................... 56

Conventions, Shading.............................. IO4

Conventional breaks............................... 6 I

Conventional lines.................................. 60

Conventional screw-threads. .......................... 62

Cross-sections................................... 62

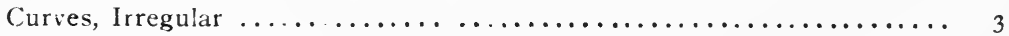

Cycloid, To describe the............................. $4^{6}$

D

Dark surfaces.................................... Io

Development of the surfaces of a hexagonal prism............... 9o

Development of the surface of a right cylinder.................. 92

Development of the surface of a cone....................... 93

Development of the surface of a cylindrical dome................. 96

Development of a locomotive gusset sheet. . . . . . . . . . . . . . 97

Dihedral angles................................... 75

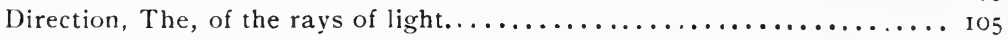

Dividers, Hair-spring.............................. 2

Drawing-board .................................. I

Drawing-pen.................................. 2

Drawing to scale $\ldots \ldots \ldots \ldots \ldots \ldots \ldots \ldots \ldots \ldots \ldots \ldots \ldots \ldots \ldots \ldots \ldots \ldots \ldots \ldots \ldots$

E

Ellipse, To describe an............................ 38

Ellipse, Given an, to find the axes and foci................... 43

Epicycloid, To describe the........................... 48

Epicycloid, To describe an interior....................... 50

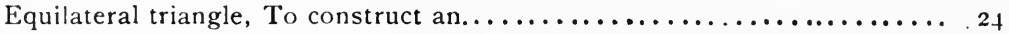

Examples of working drawings. ........................ I 20

Figuring and lettering. ............................. 66

Finished parts of working drawings $\ldots \ldots \ldots \ldots \ldots \ldots \ldots \ldots \ldots \ldots \ldots \ldots \ldots$

$\mathbf{G}$

Geometrical drawing. ................................. 16

Glass-paper pencil sharpener............................ 4

Gothic letters. ...................................... 69 
H

Heptagon, To construct a.............................. ${ }_{28}$

Hyperbola, To draw an .............................. 42

Hypocycloid, To describe the........................... $4_{48}$

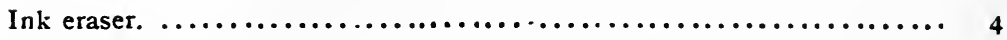

Inks............................................... 4

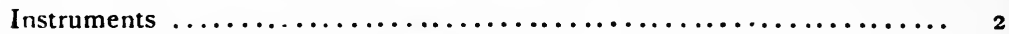

Intersection, The, of a cylinder with a cone................ 93

Intersection, The, of two cylinders...................... 96

Intersection, The, of a plane with an irregular surface of revolution...... I02

Involute, of a circle, To construct the................... 45

Isometrical cube............................... I 3

Isometrical drawing. .............................. 12

Isometrical drawing, Direction of the rays of light in. $\ldots \ldots \ldots \ldots \ldots \ldots 114$

Isometrical drawing of a two-armed cross . . . . . . . . . . . . $\ldots \ldots$

Isometrical drawing of a hollow cube $\ldots \ldots \ldots \ldots \ldots \ldots \ldots \ldots \ldots \ldots \ldots$

Isometrical drawing, Examples of $\ldots \ldots \ldots \ldots \ldots \ldots \ldots \ldots \ldots \ldots \ldots \ldots \ldots \ldots$

Isometrical scale, The $\ldots \ldots \ldots \ldots \ldots \ldots \ldots \ldots \ldots \ldots \ldots \ldots \ldots \ldots \ldots \ldots \ldots \ldots \ldots$

L

Leads for compass.................................. I $_{3}$

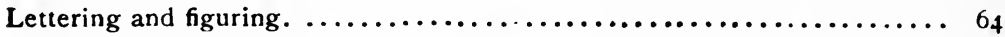

Line of shade................................... 106

Line, To draw a, parallel to another.................... 19

Line, To divide a............................... 2 1

Line of motion................................... 60

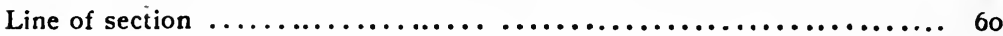

M

Mechanical drawing and elementary machine design.............. I 122

Model of the co-ordinate planes $\ldots \ldots \ldots \ldots \ldots \ldots \ldots \ldots \ldots \ldots \ldots \ldots \ldots 8_{1}$

Moulding, The "Scotia".............................. ${ }_{51}$

Moulding, The "Cyma Recta" ......................... $5^{\text {I }}$

Moulding, The "Cavetto" or "Hollow ".................... 5I $^{\text {I }}$

Moulding, The "Echinus, " "Quatrefoil," or "Ovolo"............. 52

Moulding, The "Apophygee" "......................... 52

Moulding, The "Cyma Reversa" ....................... 52

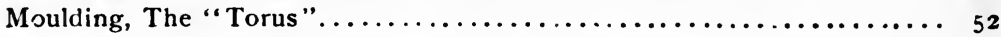


Needles $\ldots \ldots \ldots \ldots \ldots \ldots \ldots \ldots \ldots \ldots \ldots \ldots \ldots \ldots \ldots \ldots \ldots \ldots \ldots,{ }_{6}^{\mathrm{PAGE}}$

Notation............................................... 80

O

Octagon, To construct an $\ldots \ldots \ldots \ldots \ldots \ldots \ldots \ldots \ldots \ldots \ldots \ldots \ldots \ldots \ldots \ldots \ldots, 28$

Ortingraphic projection................................... 74

Oval, To construct an................................... 43

Paper...............................................

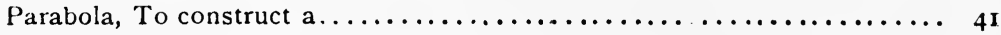

Pencil.............................................. 2

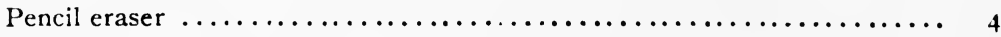

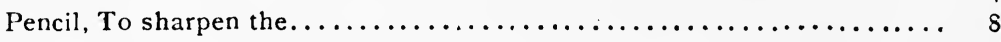

Pen, Drawing....................................... 9

Pen, To sharpen the drawing............................... 10

Pentagon, To construct a................................ 28

Perpendicular, To erect a............................ 17

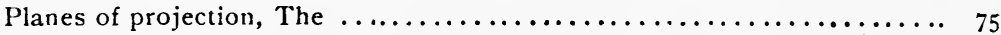

Polygon, To construct a. .............................. 26

Projection, The, of straight lines........................... 82

Projection, The, of plane surfaces.......................... 84

Projection, The, of solids............................... go

Projection, The, of the cone............................. 93

Projection of the helix as applied to screw-threads................ 99

Proportional, To find a mean, to two given lines.................. 3r

Proportional, To find a third, to two given lines................. $3^{\mathrm{I}}$

Proportional, To find a fourth, to three given lines............... 32

Protractor.............................................. 6

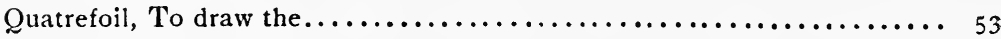

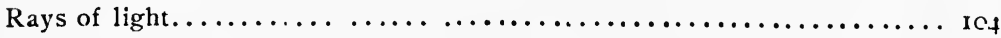

Rays, Visual........................................ 10,

Rhomboid, To construct the $\ldots \ldots \ldots \ldots \ldots \ldots \ldots \ldots \ldots \ldots \ldots \ldots \ldots \ldots \ldots \ldots \ldots$ 2

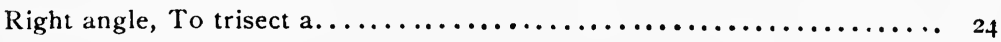

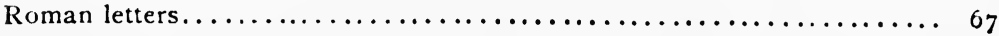




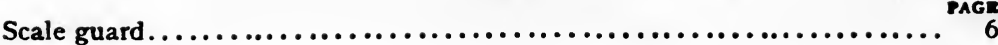

Scale, Drawing to................................... 12, 54

Scale, To construct a........................................ 55

Schiele's curve, To draw..................................... 50

Screw-threads, Conventional............................... 62

Screw-threads, Regular..................................... 100

Section lines............................................. ${ }_{56}$

Section lines, Standard................................... ${ }_{5}^{8}$

Shade lines and shading................................. 103

Shade, To, the elevation of a sphere... ..................... 108

Shade, To, a right cylinder................................ 109

Shade, To, a right cone................................. I

Shade, To, a concave cylindrical surface $\ldots \ldots \ldots \ldots \ldots \ldots \ldots \ldots \ldots \ldots$ ino

Shadows ............................................ II

Sharpen pencil, To...................................... 8

Sharpen pen, To.................................... 10

Sheet brass........................................... 6

Sheet celluloid.......................................... 6

"Sibley College" set of irregular curves.......................... 3

"Sibley College" set of instruments............................ 2

Source of light........................................ 104

Spiral, To describe the $. \ldots \ldots \ldots \ldots \ldots \ldots \ldots \ldots \ldots \ldots \ldots \ldots \ldots \ldots, 44$

Sponge rubber......................................... 5

Square, To construct a................................. 25

Stippling $\ldots \ldots \ldots \ldots \ldots \ldots \ldots \ldots \ldots \ldots \ldots \ldots \ldots \ldots \ldots \ldots \ldots \ldots, 109$

Tacks.................................................. ${ }_{5}$

T-square.............................................. 2

Third dihedral angle...................................... 75

Tinting brush $\ldots \ldots \ldots \ldots \ldots \ldots \ldots \ldots \ldots \ldots \ldots \ldots \ldots \ldots \ldots \ldots \ldots,{ }_{5}$

Tinting saucer............................................ 5

Title, The, of a working drawing........................... I22

Tracing-cloth. . ........................................... 6

Trefoil, To describe the................................... 53

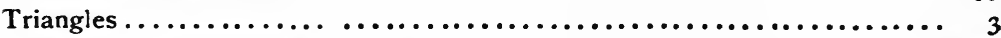

Triangle, To construct a.................................. ${ }_{25}$

Triangular scale............................................ 3

Type specimens.......................................... 70

U

Use of instruments........................................ 7

Use of pencil.............................................. 8 


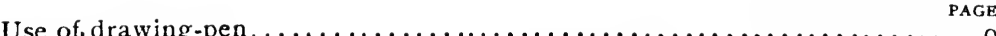

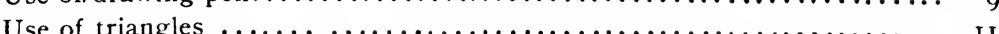

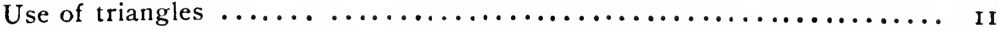

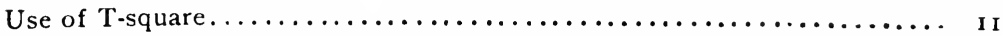

Use of drawing-board................................. II

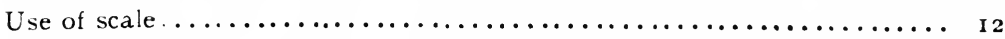

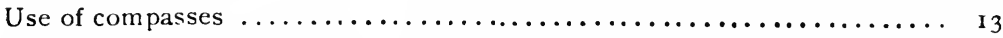

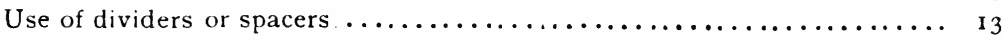

Use of spring bows........................... I4

Use of irregular curves.......................... I4

Use of protractor. $\ldots \ldots \ldots \ldots \ldots \ldots \ldots \ldots \ldots \ldots \ldots \ldots \ldots \ldots \ldots \ldots \ldots \ldots$

V

Visual rays................................... 104

Volute, To describe the "Ionic "........................ 45

W

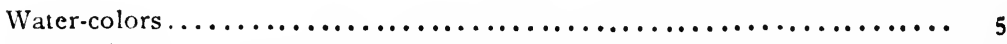

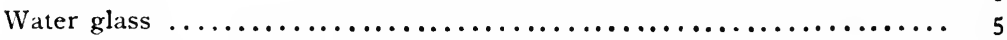

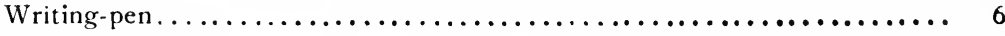

Working drawings. .............................. I 8

Working drawings, Method of making.................... II9

Working drawing, What is a . . . . . . . . . . . . . . . . . . . . I

IVorking drawings, Examples of.......................... I19

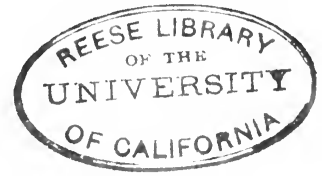






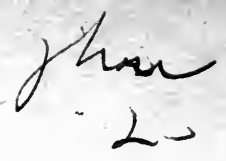




\section{THIS} STAMPED THE LAST DATE

AN

WILL BE ASSESSEDINE OF

THIS BOOKSESSED FOR OF 25

WILL INK ON THE DAT FAILURE CENTS

DAY INCREASE TO DO TE DUE. THE RETURN OVERDUE. TO \$1.00 ON ONTS ON THE FOURTY

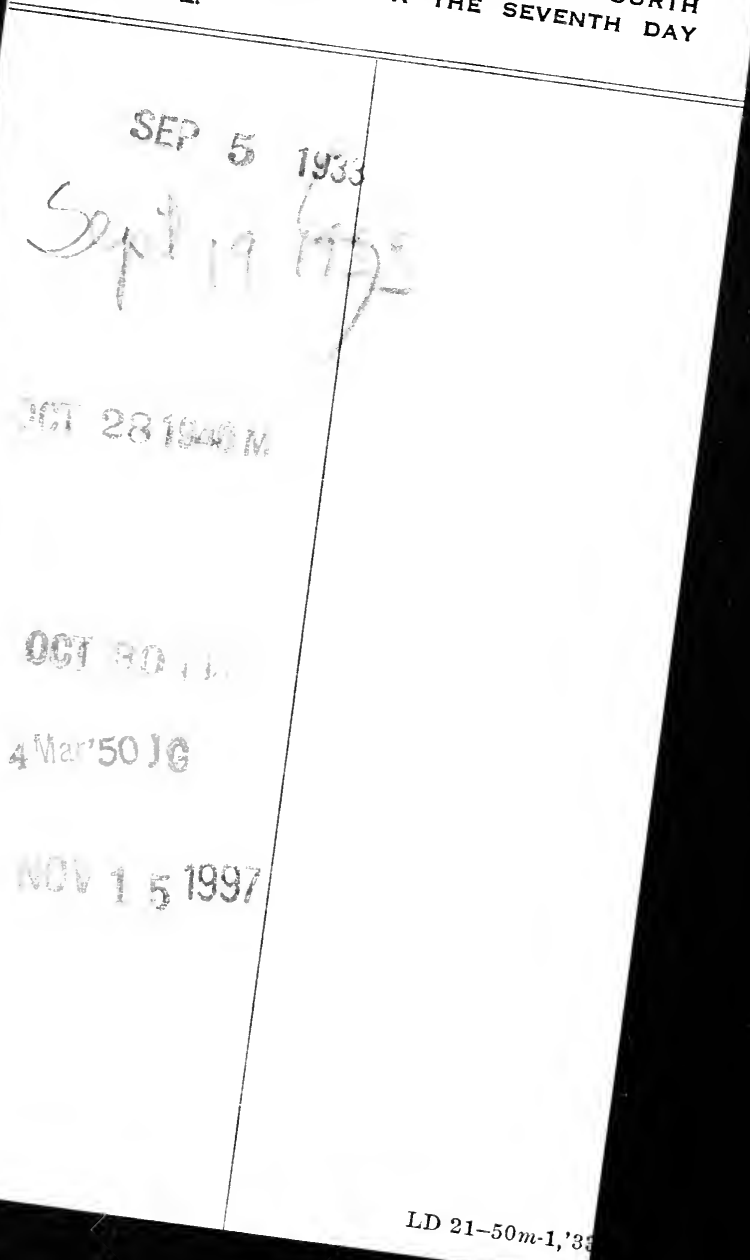



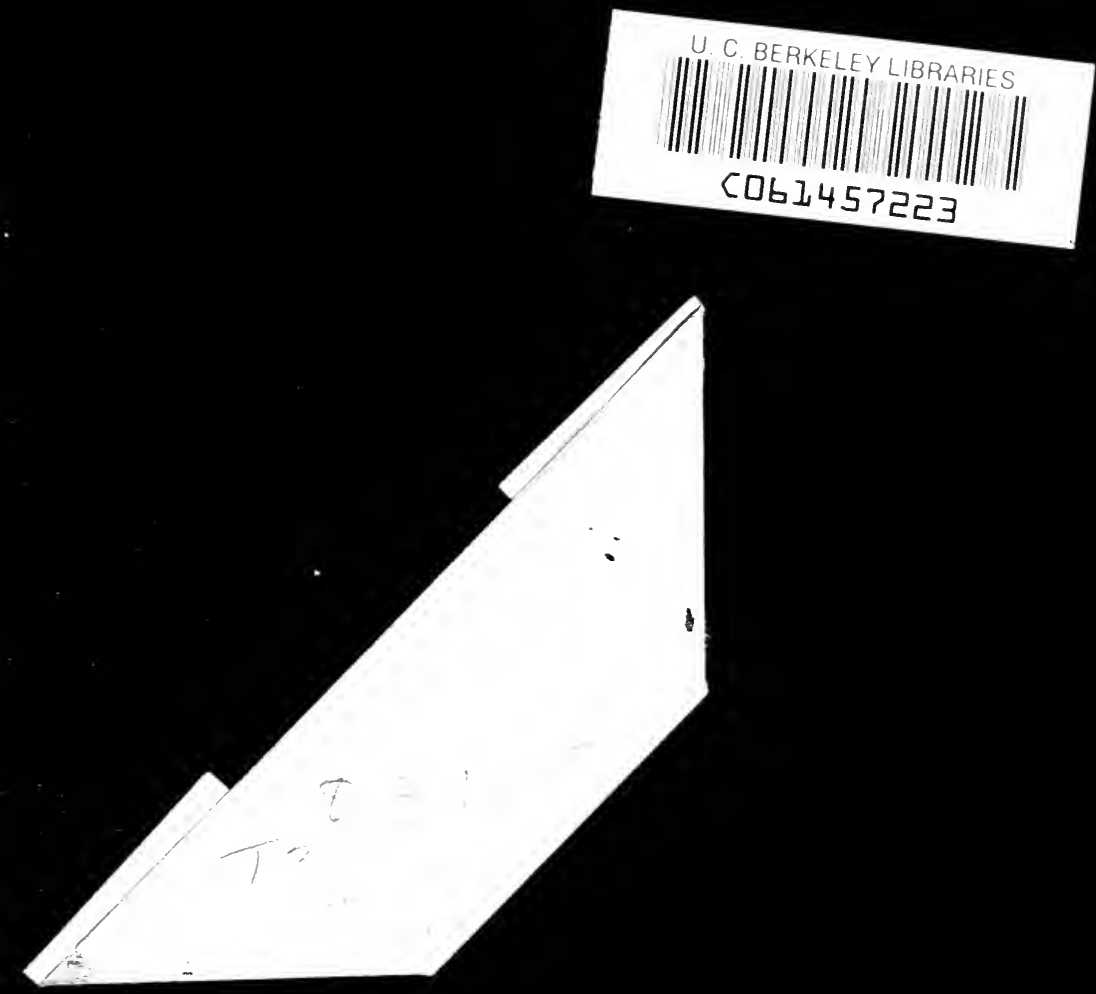
